Universidade de São Paulo

Instituto de Física

\title{
Procura de Novos Bósons Vetoriais no LHC
}

\author{
Gabriel Chicca Santucci
}

Orientador: Prof. Dr. Oscar J. P. Éboli

Dissertação apresentada ao Instituto de Física para a obtenção do título de Mestre em Ciências

Comissão examinadora:

Prof. Dr. Gustavo Alberto Burdman (IF-USP)

Profa. Dra. Marina Nielsen (IF-USP)

Prof. Dr. Sérgio Ferraz Novaes (IFT-UNESP)

São Paulo 
FICHA CATALOGRÁFICA

Preparada pelo Serviço de Biblioteca e Informação do Instituto de Física da Universidade de São Paulo

Santucci, Gabriel Chicca

Procura de novos bósons vetoriais no LHC. - Sāo Paulo, 2012.

Dissertaçāo (Mestrado) - Universidade de Sāo Paulo. Instituto de Física - Depto. de Física Matemática

Orientador: Prof. Dr. Oscar José Pinto Éboli

Área de Concentraçāo: Física

Unitermos: 1.Física de Partículas; 2. Bósons;

3. Subestrutura de Jatos.

USP/IF/SBI-059/2012 


\section{Agradecimentos}

Agradeço primeiramente a Deus. Agradeço também a meus pais Gil e Priscila, que me apoiaram de maneira imensurável durante todos meus anos de estudo e de minha vida, a eles dedico essa dissertação (ainda que não entendam nada). A meu irmão Guilherme, pelas horas gastas em frente ao video game. Aos amigos de graduação pelo companheirismo, discussões e procrastinação, principalmente ao Thaenan e ao Petre pela amizade. Aos amigos que enfrentaram a pós-graduação comigo, Bruno, João, André, Paulo, Lucas e Henrique, pelo conhecimento compartilhado nas várias discussões e pelo conhecimento que deixei de aprender quando estava jogando conversa fora.

Ao Prof. Oscar Éboli, pela oportunidade, orientação e discussões nas quais pude aprender muito do que sei hoje. Ao grande amigo Cedrick, cuja amizade construi ao longo do Mestrado, agradeço pela inúmeras discussões sobre os mais variados assuntos, mas principalmente pela ajuda no projeto, pelos cafés e pelos chocolates. Aos colegas de corredor, aos professores que me ensinaram muito ao longo do curso e a todo o pessoal do Departamento por providenciarem um ótimo ambiente de estudo e trabalho. À Aymê, por tudo.

E finalmente à Coordenação de Aperfeiçoamento de Pessoal de Nível Superior (CAPES) e à Fundação de Amparo à Pesquisa do Estado de São Paulo (FAPESP) pela bolsas concedidas. 


\section{Resumo}

Neste trabalho estudou-se a fenomenologia da observação de novos bósons vetoriais com massa da ordem de $1 \mathrm{TeV}$ no LHC. Em particular, a análise foi feita para um bóson vetorial neutro que se acopla com os bósons carregados do Modelo Padrão e esses bósons decaem hadronicamente deixando uma assinatura com jatos no detector. Esse tipo de sinal possui um enorme fundo de QCD devido ao ambiente hadrônico intrínseco ao LHC, de maneira que o uso de técnicas de análise de subestrutura de jatos se tornam indispensáveis.

A motivação do trabalho vem dos modelos com dimensões extras curvas que possuem torres de Kaluza-Klein de novos bósons de gauge. Estes são responsáveis por garantir a unitariedade nos espalhamentos dos bósons vetoriais do MP, estudamos em especial a primeira excitação do $Z^{0}$, o bóson $Z^{\prime}$. Na análise fenomenológica verificamos que o LHC possui um grande potencial de descoberta e exclusão dessas classes de modelos além do MP. Para tanto estudamos o sinal $p p \rightarrow Z^{\prime} \rightarrow W W \rightarrow$ jatos e os processos do MP do tipo $p p \rightarrow$ jatos, com pelo menos dois jatos na janela de massa do $W$. Verificamos que para um $Z^{\prime}$ de largura $\frac{M_{Z^{\prime}}}{10}$, com massa da ordem de $1 \mathrm{TeV}$ e acoplamentos da ordem de $10^{3}$ vezes menores que os acoplamentos do MP é possível observá-lo no LHC com $30 \mathrm{fb}^{-1}$ de luminosidade integrada com $14 \mathrm{TeV}$ de energia no centro de massa. 


\section{Abstract}

In this work we present a phenomenological study of the observation of new vector bosons with mass around $1 \mathrm{TeV}$ at the LHC. We analyse a neutral vector boson that couples to the charged bosons of the Standard Model and this bosons decay hadronically leaving a multiple jets signature on the detector. This kind of signal has an enormous QCD background due to the hadronic enviroment of the LHC, making the analysis of jet substruture indispensable.

The motivation for this work comes from warped extra dimensions models, wich have Kaluza-Klein towers of new gauge bosons. These are responsable for assuring the unitarity of the vector bosons scatterings in the SM. We studied the first excitation of the $Z^{0}$ bosons, the $Z^{\prime}$ boson. We verified that the LHC has a great potential of discovery or exclusion for this classes of theories beyond the SM. For the analysis, we studied the process $p p \rightarrow Z^{\prime} \rightarrow W W \rightarrow$ jets and the background processes of the SM of the type $p p \rightarrow$ jets, with at least two jets in the $W$ mass window. We verified that for a $Z^{\prime}$ with width $\frac{M_{Z^{\prime}}}{10}$, mass of $\mathcal{O}(1 \mathrm{TeV})$ and couplings $10^{3}$ times more weakly than those of the SM , it is possible to observe it in the LHC with $30 \mathrm{fb}^{-1}$ of integrated luminosity in the $14 \mathrm{TeV}$ run. 


\section{Sumário}

1 Introdução 1

2 Teorias de Calibre e Quebra Espontânea de Simetria 4

2.1 Teorias de Gauge Abelianas . . . . . . . . . . . . . . . . . . . . 4

2.2 Teorias de Gauge Não-Abelianas . . . . . . . . . . . . . . . . . . 6

2.3 Quebra Espontânea de Simetria . . . . . . . . . . . . . . . . . 9

2.3.1 Quebra Espontânea de uma Simetria Discreta . . . . . . . . . . 9

2.3.2 Quebra Espontânea de uma Simetria Contínua . . . . . . . . . . 11

2.4 O Mecanismo de Higgs no caso Abeliano . . . . . . . . . . . . . . . . . 13

3 O Modelo Padrão $\quad 15$

3.1 Fenomenologia . . . . . . . . . . . . . . . . 15

3.2 Setor de Quebra Espontânea de Simetria . . . . . . . . . . . . . . 17

3.2.1 Massa dos Bósons EletroFracos . . . . . . . . . . . . . . 19

3.3 Setor de Férmions . . . . . . . . . . . . . . . . . . . . . . 21

3.3.1 Massa dos Férmions . . . . . . . . . . . . . . . . 21

3.3.2 Termos Cinéticos Fermiônicos . . . . . . . . . . . . . . 22

3.3.3 Acoplamentos da QCD . . . . . . . . . . . . . 23

3.3.4 Acoplamentos com os Bósons de Gauge EletroFracos . . . . . . . . 24

3.4 Setor de Gauge . . . . . . . . . . . . . . . . . . . . 25

3.4.1 Interação entre os bósons de gauge eletrofracos . . . . . . . . . . 25

3.5 Autoestados de Massa, Autoestados de Gauge e a Matriz CKM . . . . . . 26

3.6 Setor de Higgs . . . . . . . . . . . . . . . . . . . . . 28

3.7 Testes do Modelo Padrão . . . . . . . . . . . . . . . . . 30

3.7.1 A Massa dos Bósons de Gauge Eletrofracos . . . . . . . . . . . . . 30

3.7.2 Assimetria na Corrente Neutra Fraca . . . . . . . . . . . . . . 31

3.7 .3 Violação de CP . . . . . . . . . . . . . . . . . . . . 32

3.7.4 Considerações Experimentais . . . . . . . . . . . . 33

3.8 Problemas com o MP . . . . . . . . . . . . . . . . 35

3.8.1 Tivialidade e Estabilidade . . . . . . . . . . . . 35

3.8 .2 Naturalidade, o Problema da Hierarquia . . . . . . . . . . . . 38 
3.8.3 Unitariedade . . . . . . . . . . . . . . . . . . . 39

3.8.4 Outras Imperfeições . . . . . . . . . . . . . . . . . . . 41

4 Introdução a Modelos com Dimensões Extras 43

4.1 Motivação . . . . . . . . . . . . . . . . . . . . 44

4.2 Modos de Kaluza-Klein . . . . . . . . . . . . . . . . . . . . 45

4.3 Dimensões Extras Curvas . . . . . . . . . . . . . . . . 46

4.3.1 Bósons de Gauge no bulk . . . . . . . . . . . . . . . 47

4.3 .2 Férmions no bulk . . . . . . . . . . . . . . . . . . 49

4.3.3 Espalhamento de Bósons de Gauge em Teorias com Dimensões Extras 51

$5 \quad$ QCD e Jatos $\quad 55$

5.1 Algoritmos de Reconstrução de Jatos . . . . . . . . . . . . . . . 57

5.1 .1 Algoritmos de Cone . . . . . . . . . . . . . . . 57

5.1 .2 Algoritmos de Recombinação . . . . . . . . . . . . . . . 58

5.1 .3 Subestrutura de Jatos . . . . . . . . . . . . . . . . 60

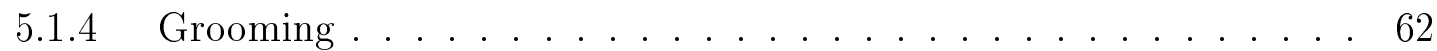

6 Simulações e Análises $\quad 65$

6.1 Motivação . . . . . . . . . . . . . . . . . . 66

6.2 Pacotes, Fundos e Cortes . . . . . . . . . . . . . 71

7 Conclusões $\quad 86$ 


\section{Lista de Figuras}

2.1 Potencial $V(\phi)$ para $\mu^{2}>0$ na esquerda e $\mu^{2}<0$ na direita. No primeiro caso há somente um mínimo global, enquanto no segundo existem dois possíveis vácuos equivalentes. . . . . . . . . . . . . . . . 10

2.2 Potencial (2.39), com $\left\langle|\phi|^{2}\right\rangle=v^{2} \ldots \ldots \ldots \ldots \ldots \ldots \ldots$. . . . . . 12

3.1 Vértices de interações entre os bósons de gauge eletrofracos. . . . . . . . 26

3.2 Auto-interações cúbica e quártica dos glúons. . . . . . . . . . . . . 26

3.3 Acoplamento entre os férmions e o bóson de Higgs. . . . . . . . . . . . . 29

3.4 Interações entre o Higgs e o W. . . . . . . . . . . . . . . . . 29

3.5 Interações entre o Higgs e o Z. . . . . . . . . . . . . . . . . 29

3.6 Poder preditivo do MP conforme medições do LEP, [23] . . . . . . . . . 33

3.7 Acoplamentos leptônicos vetorial e axial, com base nos dados do LEP, [23]. 34

3.8 Relação entre a massa do $W$ e o parâmetro $\rho,[23] . \ldots . . . .35$

3.9 Regiões excluídas pelo LEP e Tevatron para a massa do bóson de Higgs. . 37

3.10 Regiões analisadas pelas colaborações ATLAS no LHC para a massa do Higgs [24]. As regiões nas quais a curva cheia está abaixo da curva tracejada são regiões de exclusão com $95 \%$ de nível de confiança. . . . . . . . . . . 37

3.11 Diagrama correspondente às correções à massa do Higgs devido às contribuições fermiônicas no loop. . . . . . . . . . . . . . . . . 38

6.1 Distribuição do momento transverso do $Z^{\prime}$ para $M_{Z^{\prime}}=1,2,3,4 \mathrm{TeV}$. Notase que para massas maiores, a distribuição se aproxima mais do zero, pois a maior parte da energia da colisão é usada para dar massa a nova ressonância sobrando pouca energia para se transformar em momento. . . . . . . . 66

6.2 Ângulo $\Delta \phi$ entre os dois jatos mais duros dos eventos de sinal $(p p \rightarrow$ $Z^{\prime} \rightarrow W W$ para $M_{Z^{\prime}}=1,2,3,4 \mathrm{TeV}$. Como esperávamos, quanto maior a massa do $Z^{\prime}$, seu momento é mais próximo de zero e a distribuição de $\Delta \phi$ entre os dois jatos (um de cada $W$ ) é mais estreita em torno de $\Delta \phi=\pi . \quad$. 67 
6.3 Distribuição do momento transverso dos dois jatos gordos encontrados nos eventos $p p \rightarrow Z^{\prime} \rightarrow W W$, para diferentes massas do $Z^{\prime}(1,2,3 \mathrm{e} 4 \mathrm{TeV})$, sendo $j_{i}$ o $i$-ésimo jato mais duro. Pode-se observar que o primeiro jato mais duro (para cada valor de $M_{Z^{\prime}}$ ) está centrado em $\frac{M_{Z^{\prime}}}{2}$ e o segundo (menos massivo) é um pouco menos duro. Para discussão vide texto. . . . 68

6.4 Massa Invariante dos dois jatos gordos mais duros em cada evento para $M_{Z^{\prime}}=1,2,3,4 \mathrm{TeV}$. As distribuições reconstroem muito bem a massa do $W$ sendo que ainda não foi imposto nenhum corte, nem mesmo que a massa de cada jato esteja na janela de massa do $W$ (por isso as distribuições se estendem por uma faixa grande de massa). . . . . . . . . . . . . . 68

6.5 Massa invariante da combinação dos dois jatos mais duros dos eventos para $M_{Z^{\prime}}=1,2,3,4 \mathrm{TeV}$. As distribuições estão centradas em $M_{Z^{\prime}}$ sem que houvesse imposição de cortes, como na figura 6.4. Esse aspecto é de fundamental importância para redução de fundos como discutido na secção

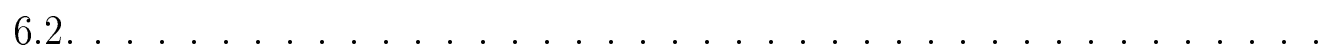

6.6 Distância $\Delta R^{2}$ entre os subjatos 1 e 2 , produzidos no decaimento $W \rightarrow j_{1} j_{2}$ para diferentes valores da massa de $Z^{\prime}$. Os subjatos que constituem o jato mais duro do evento são mostrados na figura (a) e o segundo mais duro em (b). Pode-se ver que a medida que $M_{Z^{\prime}}$ aumenta, a distância entre os jatos fica cada vez menor (mais colimados) como se espera, dificultando muito a detecção dos jatos individualmente. . . . . . . . . . . . . . . . . .

6.7 Distribuição da pseudorapidez do primeiro (a) e segundo (b) jatos mais duros nos eventos para diferentes massas de $Z^{\prime}$. A medida que $M_{Z^{\prime}}$ aumenta, as distribuições ficam mais estreitas, se concentrando na região central do detector. . . . . . . . . . . . . . . . . . . .

6.8 Distribuição do $p_{t}$ dos dois jatos mais duros nos eventos $p p \rightarrow j j$ com os eventos gerados com momento transverso mínimo: $p_{t}^{\min }=20 \mathrm{GeV}$. Vêse que a maior parte dos eventos são gerados com $p_{t}$ baixos que não irão reconstruir massas da ordem de $1 \mathrm{TeV}$. . . . . . . . . . . . . . .

6.9 Distribuição do momento transverso dos dois jatos mais duros nos eventos $p p \rightarrow j_{1} j_{2}$ e a Massa Invariante da recombinação dos dois jatos duros. Em cada figura está representado o $p_{t}^{\text {min }}$ usado na geração dos eventos para que se pudesse reconstruir a massa invariante desejada.

6.10 Distribuição do momento transverso dos dois jatos mais duros nos eventos $p p \rightarrow W^{+} W^{-} \rightarrow j j j j$ e a Massa Invariante da recombinação dos dois jatos duros. Em cada figura está representado o $p_{t}^{\text {min }}$ usado na geração dos eventos para que se pudesse reconstruir a massa invariante desejada. . . . . 
6.11 Distribuição do momento transverso dos dois jatos mais duros nos eventos $p p \rightarrow j_{1} W^{+} \rightarrow j_{1} j j$ e a Massa Invariante da recombinação dos dois jatos duros. Em cada figura está representado o $p_{t}^{\text {min }}$ usado na geração dos eventos para que se pudesse reconstruir a massa invariante desejada. . . . . 76

6.12 Distribuição do momento transverso dos dois jatos mais duros nos eventos $p p \rightarrow t \bar{t} \rightarrow b \bar{b} j j j j$ e a Massa Invariante da recombinação dos dois jatos duros. Em cada figura está representado o $p_{t}^{\text {min }}$ usado na geração dos eventos para que se pudesse reconstruir a massa invariante desejada. . . . . 77

6.13 Distribuição do momento transverso dos dois jatos mais duros nos eventos e a Massa Invariante da recombinação dos dois jatos duros. Em cada figura está representado o $p_{t}^{\text {min }}$ usado na geração dos eventos para que se pudesse reconstruir a massa invariante desejada. Os eventos são: (a) $p p \rightarrow j_{1} W^{-} \rightarrow j_{1} j j$ para $2 \mathrm{TeV}$; (b) $p p \rightarrow Z Z \rightarrow j j j j$ para $3 \mathrm{TeV}$; e (c) $p p \rightarrow j_{1} Z \rightarrow j_{1} j j$ para $4 \mathrm{TeV} \ldots \ldots \ldots \ldots . \ldots \ldots$

6.14 Parâmetros $\mu$ (a) e $y^{2}$ (b) do sinal: $Z^{\prime} \rightarrow W W$ e os processos do MP: $p p \rightarrow j j, j W, W W$ e $t \bar{t}$ para $M_{Z^{\prime}}=2 \mathrm{TeV} \ldots \ldots \ldots \ldots . \ldots . \ldots . \ldots 1$

6.15 Parâmetro $\mu$ em escala logaritmica do primeiro (a) e segundo (b) jatos mais duros dos eventos para $M_{Z^{\prime}}=2 \mathrm{TeV} \ldots \ldots \ldots \ldots$. . . . . . . 82

6.16 Parâmetro $y^{2}$ em escala logaritmica do primeiro (a) e segundo (b) jatos mais duros dos eventos para $M_{Z^{\prime}}=2 \mathrm{TeV}$. Nas figuras há um aumento na região entre 0 e 0.1 para explicitar o comportamento dos jatos de QCD

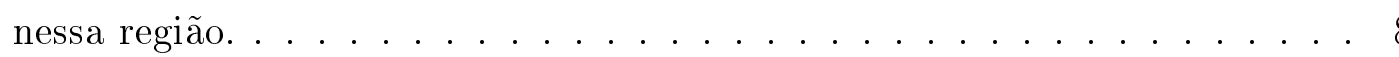




\section{Lista de Tabelas}

3.1 Números quânticos associados ao grupos $S U(3)_{C}, S U(2)_{L}$ e $U(1)_{Y}$. . . . 23

3.2 Valores de $g_{V}$ e $g_{A}$ para os férmions. . . . . . . . . . 25

6.1 Corte no $p_{t}$ dos jatos no processo $p p \rightarrow j j$ para que a geração de eventos ocorra na região de interesse. . . . . . . . . . . . . . 78

6.2 Corte no $p_{t}$ dos jatos no processo $p p \rightarrow V V \rightarrow j j j j$, onde $V=W, Z$ para que a geração de eventos ocorra na região de interesse. . . . . . . . . . . 79

6.3 Corte no $p_{t}$ dos jatos no processo $p p \rightarrow j_{1} V \rightarrow j_{1} j j$, onde $V=W, Z$ para que a geração de eventos ocorra na região de interesse. . . . . . . . . . 79

6.4 Corte no $p_{t}$ dos jatos no processo $p p \rightarrow t \bar{t} \rightarrow b \bar{b} j j j j$, para que a geração de eventos ocorra na região de interesse. . . . . . . . . . . . . 79

6.5 Momento transverso mínimo dos jatos para que sejam candidatos a um jato de $W$ para diferentes massas do $Z^{\prime}$. . . . . . . . . . . . . . 81

6.6 Eficiência e secção de choque para $M_{Z^{\prime}}=1 \mathrm{TeV}$. . . . . . . . . . . 83

6.7 Eficiência e secção de choque para $M_{Z^{\prime}}=2 \mathrm{TeV}$. . . . . . . . . . 83

6.8 Eficiência e secção de choque para $M_{Z^{\prime}}=3 \mathrm{TeV}$. . . . . . . . . . . 83

6.9 Eficiência e secção de choque para $M_{Z^{\prime}}=4 \mathrm{TeV}$. . . . . . . . . . . 84

6.10 Acoplamentos do $Z^{\prime}$ com o $W$ e os quarks do MP usados nas simulações. . 84

6.11 Valores de $\xi$ para $\mathcal{L}=30 \mathrm{fb}^{-1}$ e $\mathcal{L}=100 \mathrm{fb}^{-1}$. . . . . . . . . . . 85

6.12 Valores de $\xi \cdot \frac{S}{B}$ para $\mathcal{L}=30 \mathrm{fb}^{-1}$ e $\mathcal{L}=100 \mathrm{fb}^{-1}$ usando os valores de $\xi$ da tabela $6.11 \ldots \ldots \ldots \ldots \ldots \ldots \ldots$ 


\section{Notação}

Algumas notações e advertências para as convenções adotadas durante todo o trabalho:

- Adota-se o Sistema Natural de Unidades, no qual: $c=\hbar=1$;

- Letras gregas são usadas nos índices de Lorentz em quadrivetores: $a^{\mu} \operatorname{com} \mu=$ 0,1,2,3 e letras latinas para índices espaciais, $a^{i}, i=1,2,3$ ou índices matriciais: $\psi^{a}, a=1, \ldots, N$, onde $N$ depende da representação do grupo de simetria, mas sempre estará claro no contexto;

- Adota-se a notação de Einstein, de modo que está implícito uma soma em índices de Lorentz covariantes e contravariantes repetidos: $a^{\mu} a_{\mu}=\sum_{\mu=0}^{3} a^{\mu} a_{\mu}=a_{\mu} a^{\mu}$. Para índices matriciais a soma também é implícita, mas sempre que haja repetição, não importando onde esteja o índice;

- A métrica de Minkowsky é : $\eta^{\mu \nu}=\operatorname{diag}(1,-1,-1,-1)$;

- A representação de Dirac para as matrizes $\gamma$ é a adotada, onde $\gamma^{5}=i \gamma^{0} \gamma^{1} \gamma^{2} \gamma^{3}$ e $\left\{\gamma^{\mu}, \gamma^{5}\right\}=0$

- Usa-se Notação de Feynman para a contração de matrizes de Dirac: $\gamma^{\mu} a_{\mu}=\not$; 


\section{Capítulo 1}

\section{Introdução}

O Modelo Padrão das partículas elementares (MP) vem sendo testado experimentalmente por diversas décadas e em todos os testes a compatibilidade entre as predições teóricas e os dados experimentais são surpreendentes. Devido ao poder preditivo da teoria, inúmeras observações experimentais puderam ser feitas para verificar a teoria, como as massas dos bósons eletrofracos, $Z^{0}$ e $W^{ \pm}$, o acoplamento destes com os férmions, a universalidade dos acoplamentos de gauge e muitas outras. Com o aumento da precisão experimental ao longo dos anos, fez-se necessário o desenvolvimento de técnicas para calcular as predições teóricas do MP com mais precisão, ou seja, levando em conta às correções radioativas. Aceleradores de partículas como o LEP no CERN e o Tevatron no FermiLab, foram essenciais para os testes do modelo e novos aceleradores, como o LHC também no CERN, poderão nos dizer ainda mais sobre ele.

Apesar do enorme sucesso experimental, existem razões para acreditarmos que o Modelo Padrão não é uma teoria final que descreve as interações de gauge forte e eletrofraca. Problemas relacionados a trivialidade, naturalidade e unitariedade da teoria levam a acreditar que o MP ainda está incompleto. Inúmeras teorias surgiram nas últimas três ou quatro décadas para tentar solucionar esses problemas, as chamadas Teorias Além do Modelo Padrão. O problema da unitariedade, por exemplo, está relacionado com o setor de quebra espontânea da simetria eletrofraca. Este setor do Modelo Padrão está longe de ser compreendido totalmente. No MP a quebra da simetria eletrofraca ocorre quando um escalar (o chamado bóson de Higgs) adquire um valor esperado de vácuo não nulo, originando assim massa para algumas partículas do modelo, este é o chamado mecanismo de Higgs. Além disso, esse bóson é o responsável por garantir a unitariedade do espalhamento de bósons vetoriais longitudinais, em particular o espalhamento $W_{L}^{+} W_{L}^{-} \rightarrow W_{L}^{+} W_{L}^{-}$. A amplitude deste espalhamento cresce com a energia, ou seja, há uma divergência na secção de choque desse processo, caso não se leve em conta a troca do bósons de Higgs. Se o Higgs do MP for muito pesado ou não existir ou ainda o setor de quebra for mais complexo que o atualmente proposto, outras partículas terão de restaurar a unitariedade desse espalhamento, em particular, novas partículas com massa da ordem de $1 \mathrm{TeV}$. 
O LHC é um acelerador hadrônico que poderá responder algumas perguntas relacionadas ao setor de quebra espontânea da simetria eletrofraca (QESEF). A questão da unitariedade do espalhamento dos bósons vetoriais longitudinais, $W_{L}^{+} W_{L}^{-} \rightarrow W_{L}^{+} W_{L}^{-}$, é uma das razões pela recorrente afirmação: "Nova física deverá ser vista no LHC". Se levarmos em conta somente os diagramas que contém os bósons $\gamma$ (fóton) e o $Z^{0}$ como mediadores do espalhamento e mais o vértice quártico do $W$ a amplitude desses espalhamentos crescerá com a energia como dito acima. Porém ao adicionarmos mais dois diagramas com a troca de uma partícula escalar, a dependência com a energia é cancelada. É assim que o bóson de Higgs restaura a unitariedade desse espalhamento no MP, mas para que isso ocorra é necessário que a massa dele seja no máximo $1 \mathrm{TeV}$ aproximadamente. Caso o Higgs não exista ou seja mais pesado do que isso, então uma outra partícula deverá aparecer para restaurar a unitariedade. De maneira que alguma física nova será vista na escala TeV.

Nas últimas décadas, novas propostas ao MP apareceram para explicar a quebra espontânea de simetria e tentar resolver algumas questões do MP, as chamadas teorias além do Modelo Padrão, entre elas podemos citar technicolor, superssimetria, little Higgs e outras. Existe ainda uma classe de modelos que propõem a existência de novas dimensões espaciais, as chamadas Teorias com Dimensões Extras. Uma previsão comum à todas as teorias com dimensões extras (DE) é a existência de novas partículas, na verdade uma torre de novas partículas associadas às partículas do MP. Para determinados cenários nos quais o Higgs não é o responsável pela restauração da unitariedade do modelo, outros bósons vetoriais fazem este papel, como por exemplo uma excitação do bóson $Z^{0}$, o chamado bóson $Z$ '.

Se a massa deste novo bóson for da ordem de $1 \mathrm{TeV}$, então os produtos do seu decaimento estarão extremamente colimados e ficam cada vez mais difíceis de serem detectados. Em geral esse novo bóson decai em dois bósons do MP através de $Z^{\prime} \rightarrow W^{+} W^{-}$, os $W^{\prime} s$ poderão decair hadronicamente ou leptonicamente. No caso totalmente hadrônico, ambos os $W^{\prime} s$ decaem em jatos, mas como eles estão muito próximos (devido a enorme massa do $Z^{\prime}$ ), então em vez de observarmos dois jatos leves o detector verá somente um jato gordo, dificultando a análise dos dados. Somente com técnicas de análise de subestrutura de jatos é possível estudar este tipo de evento.

Nesta dissertação, analisamos a produção da nova ressonância $Z^{\prime}$ decaindo em $W W$, e estes decaindo hadronicamente. Mostra-se que apesar do grande fundo do Modelo Padrão para esse tipo de sinal, é possível ainda observar o novo bóson utilizando as técnicas de análise de subestrutura aqui apresentadas.

Nos primeiros capítulos apresenta-se uma breve revisão de assuntos introdutórios da física de partículas, que são essenciais para o entendimento das teorias com nova física. No capítulo 2 introduz-se os conceitos de Simetria de Calibre e Quebra Espontânea de Simetria para discutir o Modelo Padrão no capítulo 3. Revisamos sua estrutura, os teste realizados e os problemas a ele relacionados. No capítulo 4 apresenta-se uma introdução 
às Teorias com Dimensões Extras e Dimensões Extras Curvas e como elas se relacionam com a questão da restauração da unitariedade.

No capítulo 5 dá-se uma visão geral e superficial dos assuntos relacionados a jatos, a saber, como se define um jato, os algoritmos de buscas e as novas técnicas de subestrutura de jatos e de refinamento das análises. No capítulo 6 explica-se as simulações realizadas, os programas e algoritmos utilizados e a apresentação dos resultados das análises. 


\section{Capítulo 2}

\section{Teorias de Calibre e Quebra Espontânea de Simetria}

O Modelo Padrão das partículas elementares (MP) é uma teoria de gauge (ou calibre) $S U(3)_{C} \times S U(2)_{L} \times U(1)_{Y}$ que apresenta a quebra espontânea da simetria $S U(2)_{L} \times$ $U(1)_{Y} \rightarrow U(1)_{E M}$. Uma teoria de gauge é uma teoria na qual existem graus de liberdade não físicos na teoria, dado um certo campo de gauge é possível fazer uma transformação para um novo campo que mantém a ação invariante, esse tipo de transformação é chamada de transformações de gauge ou de calibre. Aqui descreve-se brevemente esses assuntos e como juntos são responsáveis pela geração de interações e massa no MP.

\subsection{Teorias de Gauge Abelianas}

Numa teoria de gauge, uma simetria global de uma lagrangeana passa a ser local quando se introduz o chamado campo de gauge. Tomemos como exemplo a teoria de Dirac para um férmion sem massa e livre, ou seja, sem interações.

$$
\mathcal{L}=i \bar{\psi} \not \partial \psi
$$

Esta lagrangeana é invariante sob uma transformação: $\psi \rightarrow \psi^{\prime}=e^{i \alpha} \psi$, onde $\alpha$ é um parâmetro global, ou seja, não depende das coordenadas $x$. Sendo assim: $\partial_{\mu} \psi \rightarrow$ $\partial_{\mu}\left(e^{i \alpha} \psi\right)=e^{i \alpha} \partial_{\mu} \psi$ e $\bar{\psi} \rightarrow \bar{\psi}^{\prime}=\bar{\psi} e^{-i \alpha}$ e a lagrangeana fica invariante.

Porém se pedirmos que $\alpha$ seja um parâmetro local, ou seja, $\alpha=\alpha(x)$ agora esse termo não é mais invariante, uma vez que

$$
\partial_{\mu}\left(e^{i \alpha} \psi\right)=e^{i \alpha}\left[\partial_{\mu} \psi+i\left(\partial_{\mu} \alpha\right) \psi\right]
$$

e

$$
\mathcal{L}^{\prime}=\mathcal{L}-\bar{\psi}(\not \partial \alpha) \psi
$$


A presença da derivada em relação às coordenadas impede que a lagrangeana (e portanto a ação) seja invariante pela transformação local. Porém essa transformação não passa de uma fase para o campo $\psi$ de modo que sua presença não deveria modificar a física contida nele. Sabe-se da mecânica quântica que a função de onda de uma partícula sempre pode ser multiplicada por uma fase sem alteração do estado físico da partícula, uma vez que somente $|\psi|$ é importante. Além disso, a localidade de $\alpha$ também é importante, diferentes observadores devem ser capazes de descrever a mesma física independente dessa fase, ou seja, o valor de $\alpha$ deve ser arbitrário para observadores em diferentes lugares do Universo. Então espera-se que $\alpha$ possa depender das coordenadas e não seja um mesmo valor universal para todos os referenciais inerciais.

Então para que $\mathcal{L}^{\prime}=\mathcal{L}$, é necessário que $\partial_{\mu} \psi$ se transforme como o próprio campo $\psi$. Para tanto, introduz-se uma derivada covariante, $D_{\mu}$ que substitui todas as derivadas parciais e satisfaz $D_{\mu} \psi \rightarrow D_{\mu}^{\prime}\left(e^{i \alpha} \psi\right)=e^{i \alpha} D_{\mu} \psi$. Tem-se que:

$$
D_{\mu} \equiv \partial_{\mu}+i e A_{\mu}
$$

e sob essa transformação, $A_{\mu}$ satisfaz:

$$
A_{\mu} \rightarrow A_{\mu}^{\prime} \equiv A_{\mu}-\frac{1}{e} \partial_{\mu} \alpha
$$

assim, a derivada covariante se transforma como o campo $\psi$. Com $e$ sendo uma constante de acoplamento (que ficará mais claro adiante) e $A_{\mu}$ um novo campo com caráter vetorial que tivemos que introduzir. Podemos ver claramente que:

$$
\begin{aligned}
\left(D_{\mu} \psi\right)^{\prime}= & \left(\partial_{\mu}+i e A_{\mu}^{\prime}\right) \psi^{\prime}=e^{i \alpha} \partial_{\mu} \psi+i e^{i \alpha}\left(\partial_{\mu} \alpha\right) \psi+e^{i \alpha} i e A_{\mu} \psi-e^{i \alpha} i e \frac{1}{e}\left(\partial_{\mu} \alpha\right) \psi= \\
= & e^{i \alpha}\left[\partial_{\mu}+i e A_{\mu}\right] \psi=e^{i \alpha} D_{\mu} \psi
\end{aligned}
$$

e assim, $(\bar{\psi} \not D \psi)^{\prime}=\bar{\psi} e^{-i \alpha} e^{i \alpha} \not D \psi=\bar{\psi} \not D \psi$ e a ação se mantém invariante sob a transformação de fase local. Pode-se reproduzir exatamente o cálculo feito aqui acrescentando-se um termo de massa para o férmion: $m \bar{\psi} \psi$. Até aqui não faz diferença, pois este termo também é invariante de gauge, mas no MP um termo de massa para os férmions é proibido como veremos, então já assumimos férmions sem massa desde o início. Vale notar porém que para o campo bosônico um termo de massa não é permitido pela invariância de gauge, uma vez que $m^{2} A_{\mu}^{\prime} A^{\prime \mu} \neq m^{2} A_{\mu} A^{\mu}$.

Para obter a invariância da lagrangeana foi necessário a introdução do campo vetorial $A_{\mu}$. Este novo campo se acopla com o férmion através de um novo termo de interação:

$$
\mathcal{L}=\mathcal{L}_{\text {Livre }}+\mathcal{L}_{\text {Int }}
$$


onde

$$
\mathcal{L}_{\text {Int }}=-e \bar{\psi} \gamma^{\mu} \psi A_{\mu} \equiv-J^{\mu} A_{\mu}
$$

A imposição da invariância de gauge introduziu naturalmente um termo de interação entre os campos. Diz-se que o campo $A_{\mu}$ é o mediador dessa interação. Identificando os campos com uma teoria conhecida, temos que o campo $\psi$ descreve um férmion de Dirac como por exemplo o életron ${ }^{1}$ e o campo $A_{\mu}$ descreve o fóton, um campo vetorial não massivo, $e$ é a carga elétrica do férmion e a receita (2.4) é a bem conhecida substituição mínima da mecânica $p_{\mu} \rightarrow p_{\mu}-e A_{\mu}$. Para montar a lagrangeana da QED basta acrescentar um termo cinético para o fóton, pois este também deve se propagar:

$$
\mathcal{L}_{Q E D}=\mathcal{L}_{\text {Dirac }}+\mathcal{L}_{\text {Maxwell }}+\mathcal{L}_{\text {Int }}
$$

com

$$
\mathcal{L}_{\text {Maxwell }}=-\frac{1}{4} F^{\mu \nu} F_{\mu \nu} \equiv-\frac{1}{4}\left(\partial^{\mu} A^{\nu}-\partial^{\nu} A^{\mu}\right)\left(\partial_{\mu} A_{\nu}-\partial_{\nu} A_{\mu}\right)
$$

Onde $F^{\mu \nu}$ é o tensor eletromagnético, este termo da lagrangeana de Maxwell é invariante de gauge e de Lorentz e por isso também deve ser adicionado à teoria ${ }^{2}$. Pode-se mostrar que as equações de movimento associadas a essa lagrangeana leva às equações de Maxwell sem fontes, veja por exemplo [1].

O exemplo usado aqui para ilustrar uma teoria de Gauge assumiu que $\alpha$ era somente um parâmetro, ou seja, o grupo associado à simetria de gauge era um grupo abeliano, em particular, $U(1)$ tal que: $e^{-i \alpha} e^{i \alpha}=1$. No modelo padrão encontraremos outros grupos de simetrias com representações unitárias que não são abelianos, ou seja, a matriz de transformação associada à simetria do grupo de gauge ainda satisfaz: $U^{-1} U=I$, mas não há comutação entre os elementos do grupo . Passamos então a generalizar a idéia dessa secção.

\subsection{Teorias de Gauge Não-Abelianas}

A generalização para teorias não-abelianas é quase imediata, bastando somente um cuidado maior nos cálculos devido à anticomutação e ao caráter matricial presente. Uma das primeiras motivações associadas às simetrias não-abelianas foi de Yang e Mills para tratar o problema de isospin nuclear. Devido à quase degenerescência entres as massas do próton e do nêutron e ao fato da interação nuclear forte não os distinguir, formulou-se uma teoria em função de uma partícula genérica, o nucleon.

Nesse tipo de teoria o campo $\psi$ é um espinor de $N$ componentes, associado ao grupo

\footnotetext{
${ }^{1}$ Basta introduzir o termo de massa na lagrangeana.

${ }^{2}$ Há ainda mais um termo invariante de Lorentz e de gauge, do tipo $\tilde{F}_{\mu \nu} F^{\mu \nu}$, com $\tilde{F}$ sendo o dual de $F$ dado por $\epsilon^{\alpha \beta \gamma \delta} F_{\alpha \beta}$. Mas esse termo dá origem a um termo de superfície que não altera a dinâmica da teoria em 4D, porém passa a ter importância em teorias de Chern-Simons.
} 
de simetria $S U(N)$. No estudo do isospin nuclear ou das interações fracas do MP tem-se $N=2$. Para a QCD, a teoria das interações fortes, $N=3$. Então os férmions de Dirac ${ }^{3}$ podem ser descritos pela lagrangeana:

$$
\mathcal{L}=i \bar{\psi} a \not \partial \psi_{a}
$$

com $a=1, \ldots, N$. Daqui para frente o índice $a$ será implícito para aliviar a notação. No caso do estudo das interações nucleares tem-se: $\mathcal{L}=i \bar{\psi} a \not \partial \psi_{a}=i \bar{p} \not \partial p+i \bar{n} \not \partial n$, com $p$ e $n$ os campos do próton e do nêutron. Queremos então que o campo $\psi$ se transforme de acordo com a simetria:

$$
\psi \rightarrow \psi^{\prime}=U(x) \psi
$$

e $U(x)=e^{i \mathbf{T} \cdot \alpha(x)}=e^{i \frac{\tau^{a}}{2} \alpha_{a}(x)}$. Sendo $\tau$, as matrizes $N \times N$ unitárias de determinante unitário que geram o grupo $S U(N)$ e o fator de 2 é para conveniência futura. Então a lagrangeana (2.11) não é invariante por (2.12), pois

$$
\partial_{\mu} \psi \rightarrow U \partial_{\mu} \psi+\left(\partial_{\mu} U\right) \psi
$$

Novamente introduz-se uma derivada covariante

$$
D_{\mu}=I \partial_{\mu}+i g B_{\mu}
$$

com $I$ a identidade $N \times N$ que escreveremos de modo implícito agora. $B_{\mu}$ é um campo vetorial não comutante (devido ao caráter não-abeliano do grupo de simetria) e $g$ é a constante de acoplamento da interação, análoga a carga elétrica da QED. Explicitando o caráter matricial de $B$ podemos escrevê-lo como $B_{\mu}=\frac{\tau}{2} \cdot b_{\mu}$ com $b_{\mu}$ os campos de gauge. No caso $S U(2)$ por exemplo, $\tau$ são as matrizes de Pauli e $B_{\mu}=\frac{1}{2}\left(\begin{array}{cc}b_{3} & b_{1}-i b_{2} \\ b_{1}+i b_{2} & -b_{3}\end{array}\right)$. Inspirado pelo caso abeliano, a transformação do campo $B_{\mu}$ é dada por:

$$
B_{\mu}^{\prime}=U B_{\mu} U^{-1}+\frac{i}{g}\left(\partial_{\mu} U\right) U^{-1}
$$

e assim, a derivada covariante satisfaz

$$
\begin{aligned}
\left(D_{\mu} \psi\right)^{\prime}= & \left(\partial_{\mu}+i g B_{\mu}^{\prime}\right) U \psi=\left(\partial_{\mu} U\right) \psi+U \partial_{\mu} \psi+i g\left[U B_{\mu}+\frac{i}{g}\left(\partial_{\mu} U\right)\right] U^{-1} U \psi= \\
= & U \partial_{\mu} \psi+i g U B_{\mu} \psi=U D_{\mu} \psi
\end{aligned}
$$

Desse modo a derivada se transforma como o campo deixando a lagrangeana invariante. Novamente, como esperávamos, um termo de massa para o campo de gauge é proibido

\footnotetext{
${ }^{3}$ Novamente, por facilidade na notação, estudaremos férmions não massivos. Mas todo o cálculo segue acrescentando um termo de massa para eles.
} 
pela simetria. Como no caso abeliano, a derivada covariante introduz uma interação entre os campos fermiônicos livres e os campos vetoriais de gauge:

$$
\mathcal{L}=\mathcal{L}_{\text {Férmions }}+\mathcal{L}_{\text {Int }}
$$

com

$$
\mathcal{L}_{\text {Int }}=-\frac{g}{2} \bar{\psi} \gamma^{\mu} \tau^{a} \psi b_{\mu}^{a}
$$

No MP os férmions estão na representação fundamental do grupo de simetria, enquanto os bósons de gauge estão na representação adjunta. O número de bósons mediadores é o número de geradores do grupo, $N^{2}-1$, no caso de um grupo $S U(N)$. Sendo que em geral, esses geradores não comutam, formando uma álgebra:

$$
\left[T^{a}, T^{b}\right]=i f^{a b c} T^{c}
$$

onde $T=\frac{1}{2} \tau$ e $f^{a b c}$ são as constantes de estrutura do grupo.

Para construir uma teoria que descreva a interação entre os férmions e os bósons, basta acrescentar o termo cinético dos bósons. Devido ao caráter anticomutante dos campos o tensor $F^{\mu \nu}$ também precisa ser generalizado. Em analogia com o caso da QED, queremos um tensor ${ }^{4} F_{\mu \nu}$ que satisfaça:

$$
F_{\mu \nu}^{\prime}=U F_{\mu \nu} U^{-1}
$$

para que a lagrangeana seja invariante sobre as transformações do grupo de gauge. Observando que no caso abeliano podemos escrever:

$$
F_{\mu \nu}=\frac{1}{i e}\left[D_{\mu}, D_{\nu}\right]=\partial_{\mu} A_{\nu}-\partial_{\nu} A_{\mu}+i e\left[A_{\mu}, A_{\nu}\right]
$$

vamos generalizar para o caso $S U(N)$, então:

$$
F_{\mu \nu}=\frac{1}{i q}\left[D_{\mu}, D_{\nu}\right]=\partial_{\mu} B_{\nu}-\partial_{\nu} B_{\mu}+i q\left[B_{\mu}, B_{\nu}\right]
$$

Uma outra forma de se obter $F_{\mu \nu}$ usando os geradores $\tau$ é:

$$
F_{\mu \nu} \equiv \frac{1}{2} \mathbf{F}_{\mu \nu} \cdot \tau=\frac{1}{2} F_{\mu \nu}^{a} \tau^{a}
$$

e com isso podemos construir um termo invariante de gauge

$$
-\frac{1}{4} \mathbf{F}_{\mu \nu} \cdot \mathbf{F}^{\mu \nu}=-\frac{1}{2} \operatorname{Tr}\left(\frac{\tau^{a}}{2} F_{\mu \nu}^{a} \frac{\tau^{b}}{2} F_{b}^{\mu \nu}\right)=-\frac{1}{2} \operatorname{Tr}\left(F_{\mu \nu} F^{\mu \nu}\right)
$$

a invariância de (2.24) é imediata usando (2.20):

\footnotetext{
${ }^{4}$ Muitas vezes chamado de curvatura, na linguagem de geometria diferencial ou ainda campo de YangMills. O campo de gauge $A_{\mu}$ é a conexão ou potencial de Yang-Mills.
} 


$$
\operatorname{Tr}\left(F_{\mu \nu}^{\prime} F^{\prime \mu \nu}\right)=\operatorname{Tr}\left(U F_{\mu \nu} U^{-1} U F^{\mu \nu} U^{-1}\right)=\operatorname{Tr}\left(F_{\mu \nu} F^{\mu \nu}\right)
$$

A principal diferença entre as teorias abelianas e não-abelianas é devido à presença do comutador não nulo entre os campos em (2.22). Por causa dele, os bósons de gauge interagem entre si, diferentemente da QED onde os fótons não interagem. O termo cinético apresenta termos cúbicos e quárticos nos campos, de modo que há vértices com 3 e 4 bósons.

Passamos agora a discutir a quebra espontânea das simetrias que acabamos de ver. Com isso teremos todas as ferramentas necessárias para construir o MP.

\subsection{Quebra Espontânea de Simetria}

Uma simetria é dita exata quando a Lagrangeana da teoria e o vácuo respeitam essa simetria. Então

$$
\delta \mathcal{L}=0
$$

e se $Q$ é o gerador da simetria e $|0\rangle$ é o vácuo, então:

$$
Q|0\rangle=0 \Rightarrow e^{i Q}|0\rangle=|0\rangle
$$

e ambos ficam invariantes pela transformação. Mas no caso em que a lagrangeana apresenta uma simetria, porém o vácuo não a respeita, diz-se que a simetria é quebrada espontaneamente. O exemplo mais comum é o ferromagneto de Heisenberg, nele os spins das partículas constituintes são aleatoriamente distribuidos. De modo que há uma simetria de rotação, nenhuma direção é privilegiada pela orientação dos spins. Porém, abaixo da temperatura de Curie os spins se alinham, minimizando a energia de interação, de modo que todos apontam para uma mesma direção. A priori esta direção é qualquer, devido à simetria inicial, mas uma vez que se atinja esse estado uma direção preferencial foi escolhida e a simetria de rotação está escondida. Vamos descrever brevemente o caso das simetrias discretas e contínuas.

\subsubsection{Quebra Espontânea de uma Simetria Discreta}

Para o caso de uma simetria discreta é fácil ver o que acontece. O exemplo canônico é o que nos servirá posteriormente como o modelo de Higgs, então tomemos um campo escalar real auto-interagente:

$$
\mathcal{L}=\frac{1}{2} \partial_{\mu} \phi \partial^{\mu} \phi-V(\phi)
$$

com

$$
V(\phi)=\frac{1}{2} \mu^{2} \phi^{2}+\frac{\lambda}{4} \phi^{4}
$$


$\operatorname{com} \lambda>0$ para garantir a estabilidade da teoria, ou seja, o potencial é limitado inferiormente fazendo com que exista um estado de menor energia. Esta lagrangeana apresenta uma simetria discreta de paridade, fazendo $\phi \rightarrow-\phi$ ela se mantém invariante. Este é o potencial mais geral possível que é renormalizável, pois potências maiores do que $\phi^{4}$ não são renormalizáveis por contagem de potência ( $\lambda$ é adimensional e $\mu$ tem dimensões de massa). Nos livros textos de campos como [2] esta teoria é conhecida como $\lambda \phi^{4}$.

- Se $\mu^{2}>0$, o mínimo do potencial é em $\phi=0$ e é único (figura 2.1(a)). Este vácuo respeita a simetria da lagrangeana e no estado de menor energia a energia cinética é nula, então

$$
\langle\mathcal{H}\rangle_{0}=\langle V\rangle_{0}=\langle\phi\rangle_{0}=0
$$

Podemos tomar

$$
\mathcal{L}=\frac{1}{2}\left[\partial_{\mu} \phi \partial^{\mu} \phi-\mu^{2} \phi^{2}\right]
$$

para pequenas oscilações em torno do vácuo e a teoria é a de uma partícula livre com $\operatorname{massa} \mu$.

- Mas se $\mu^{2}<0$ temos uma situação de quebra espontânea de simetria, pois o vácuo não mais respeita a simetria de paridade. Neste caso, o mínimo ocorre para

$$
\langle\phi\rangle_{0}= \pm \sqrt{\frac{-\mu^{2}}{\lambda}} \equiv \pm v
$$

sendo $v$ o valor esperado do vácuo $(\mathrm{VEV})$ da teoria. Aqui há dois estados de mínima energia como pode-se ver na figura 2.1(b), diz-se que o vácuo é degenerado.

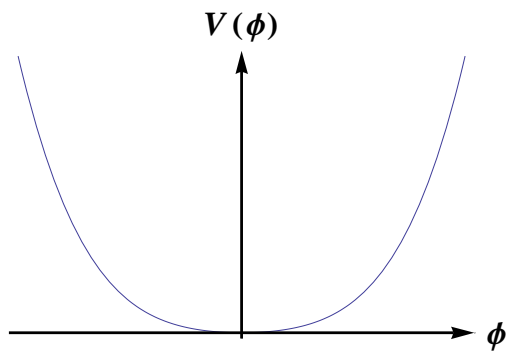

(a)

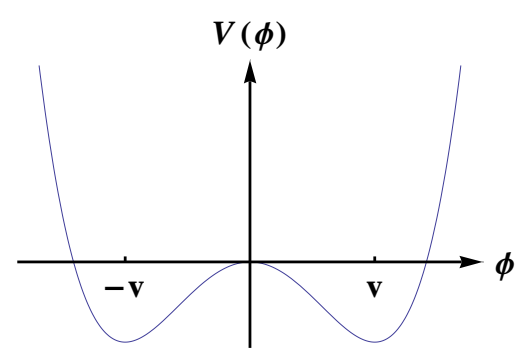

(b)

Figura 2.1: Potencial $V(\phi)$ para $\mu^{2}>0$ na esquerda e $\mu^{2}<0$ na direita. No primeiro caso há somente um mínimo global, enquanto no segundo existem dois possíveis vácuos equivalentes.

Ambos os vácuos são equivalentes, mas é necessário que se escolha um como o vácuo da teoria e então quebra-se a simetria. Escolhendo $\langle\phi\rangle_{0}=+v$, podemos deslocar o zero do potencial redefinindo o campo $\phi$

$$
\phi^{\prime}=\phi-\langle\phi\rangle_{0}=\phi-v \Rightarrow\left\langle\phi^{\prime}\right\rangle_{0}=0
$$


Reescrevendo a lagrangeana em função do novo campo o termo cinético permanece igual (pois só acrescentamos uma constante), mas o novo potencial fica (lembrando que $\left.\lambda v^{2}=-\mu^{2}\right)$

$$
V=-\mu^{2}\left[\frac{\phi^{\prime 4}}{4 v^{2}}+\frac{\phi^{\prime 3}}{v}+\phi^{\prime 2}-\frac{v^{2}}{4}\right]
$$

onde fica claro que a simetria inicial de paridade foi perdida. Para pequenas oscilações podemos aproximar $V$ por um potencial quadrático e então

$$
\mathcal{L}=\frac{1}{2}\left[\partial_{\mu} \phi \partial^{\mu} \phi+2 \mu^{2} \phi^{2}\right]
$$

que descreve uma partícula livre com massa $m^{2}=-2 \mu^{2}>0$.

\subsubsection{Quebra Espontânea de uma Simetria Contínua}

Para uma simetria contínua ${ }^{5}$ o raciocínio é análogo mas o resultado é mais interessante. Considere o mesmo caso anterior, mas agora com dois campos escalares reais que formam um dubleto de $S O(2)$.

$$
\phi \equiv\left(\begin{array}{l}
\phi_{1} \\
\phi_{2}
\end{array}\right)
$$

O termo cinético dos dois campos

$$
\mathcal{L}_{\text {cin }}=\frac{1}{2}\left(\partial_{\mu} \phi_{1} \partial^{\mu} \phi_{1}+\partial_{\mu} \phi_{2} \partial^{\mu} \phi_{2}\right)
$$

pode ser reescrito com a ajuda da notação matricial, $\phi^{\dagger}=\left(\begin{array}{ll}\phi_{1} & \phi_{2}\end{array}\right)$, então:

$$
\mathcal{L}=\frac{1}{2} \partial_{\mu} \phi^{\dagger} \partial^{\mu} \phi-V\left(\phi^{\dagger} \phi\right)
$$

com

$$
V\left(\phi^{\dagger} \phi\right)=\frac{1}{2} \mu^{2}\left(\phi^{\dagger} \phi\right)+\frac{\lambda}{4}\left(\phi^{\dagger} \phi\right)^{2}
$$

neste caso, há uma simetria de rotação, sendo a lagrangeana invariante pela transformação:

$$
\phi \rightarrow \phi^{\prime}=U(x) \phi
$$

sendo $U(x)$, uma matriz de rotação $2 \times 2$.

- Se $\mu^{2}>0$ o caso é completamente análogo ao caso discreto, com duas partículas auto-interagentes, mas que não interagem entre si e possuem uma mesma massa $\mu$

\footnotetext{
${ }^{5}$ Uma simetria é dita contínua quando pode ser parametrizada por um parâmetro contínuo, como um ângulo de uma rotação.
} 
(pois estão num mesmo dubleto).

- Mas no caso $\mu^{2}<0$ o resultado é diferente. O mínimo do potencial ocorre para

$$
\left\langle\phi^{\dagger} \phi\right\rangle_{0}=\frac{-\mu^{2}}{\lambda} \equiv v^{2}
$$

que corresponde a infinitos vácuos degenerados devido a simetria contínua de rotação. O potencial é chamado de chapéu mexicano ou fundo de garrafa devido ao seu formato como na figura 2.2, basta girar o potencial da figura 2.1 (b) em torno do eixo y.

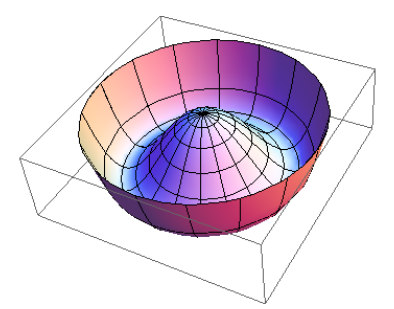

Figura 2.2: Potencial (2.39), com $\left\langle|\phi|^{2}\right\rangle=v^{2}$.

Assim como no caso discreto é necessário escolher um dos vácuos e assim quebrar a simetria. Qualquer escolha que satisfaça $\phi_{1}^{2}+\phi_{2}^{2}=v^{2}$ é equivalente. Escolhendo então

$$
\langle\phi\rangle_{0}=\left(\begin{array}{l}
v \\
0
\end{array}\right)
$$

e redefinindo o campo $\phi$ tem-se

$$
\phi^{\prime}=\phi-\langle\phi\rangle_{0}=\left(\begin{array}{c}
\phi_{1}-v \\
\phi_{2}
\end{array}\right) \Rightarrow\left\langle\phi^{\prime}\right\rangle_{0}=\left(\begin{array}{l}
0 \\
0
\end{array}\right)
$$

com a nova definição o termo cinético ainda é o mesmo, porém o potencial toma a nova forma

$$
V=-\mu^{2} \phi_{1}^{2}+\mu^{2} v \phi_{1}+\frac{\lambda}{4}\left(\phi^{\dagger} \phi\right)^{2}-\lambda v \phi_{1}\left(\phi^{\dagger} \phi\right)
$$

Vemos que o potencial não é mais invariante sob a transformação (2.40) e que o campo $\phi_{1}$ adquiriu um termo de massa mas no entanto $\phi_{2}$ continua não massivo. Olhando o potencial da figura 2.2 vemos que a transformação (2.40) conecta os infinitos vácuos. Na direção angular, não há custo de energia, de modo que o campo $\phi_{2}$ não ganha massa. Porém na direção radial existe o custo e $\phi_{1}$ adquire uma massa $m^{2}=-\mu^{2}>0$.

Esse resultado é um caso particular do chamado Teorema de Goldstone. Um bóson não massivo, conhecido como bóson de Goldstone aparece para cada gerador da simetria que foi quebrado. No caso apresentado, só havia um gerador quebrado e $\phi_{2}$ é o bóson de Goldstone. 
Vimos então que para teorias de Yang-Mills a simetria de calibre não permite colocar um termo de massa para os bósons de gauge, pois esse não é invariante de gauge. Vimos também que quando quebramos uma simetria contínua espontaneamente aparecem bósons não massivos na teoria, os bósons de Goldstone. O chamado Mecanismo de Higgs faz com que haja uma cooperação entre os dois casos unindo a quebra espontânea de simetria e as teorias de Yang-Mills.

\subsection{O Mecanismo de Higgs no caso Abeliano}

O Mecanismo de Higgs consiste em unir uma teoria de Yang-Mills e a quebra espontânea de simetria. Na seç̧ão anterior vimos como funciona o mecanismo de quebra para uma simetria contínua, como por exemplo um rotação. Aqui procederemos da mesma forma, mas estudando o que ocorre na quebra de uma simetria de gauge numa teoria de YangMills. Por facilidade didática trataremos do exemplo abeliano, cuja simetria de gauge é dada pelo grupo abeliano $U(1)$. O caso não-abeliano é o que aparece no MP, então faremos esse caso com mais detalhes utilizando diretamente como exemplo o caso do MP na secção 3.2 .

Considere então uma lagrangeana que descreve um campo escalar $\phi$ e sua interação com um bóson vetorial $A_{\mu}$ :

$$
\mathcal{L}=D_{\mu} \phi^{\dagger} D^{\mu} \phi-\frac{1}{2} \mu^{2}\left(\phi^{\dagger} \phi\right)-\frac{\lambda}{4}\left(\phi^{\dagger} \phi\right)^{2}-\frac{1}{4} F_{\mu \nu} F^{\mu \nu}
$$

onde vemos o termo cinético dos campos $\phi$ e $A_{\mu}$, um potencial de interação do campo $\phi$ dado por (2.39) e a derivada covariante dada por (2.4) que garante a invariância de gauge. Como foi visto na secção 2.1, essa lagrangeana é invariante por transformações de fase, $\phi^{\prime} \rightarrow e^{i \alpha(x)} \phi$ com o parâmetro $\alpha$ dependendo das coordenadas. Como antes ${ }^{6}$ vamos supor $\lambda>0$ para que haja estabilidade na teoria e $-\mu^{2}>0$ para que o potencial tenha um mínimo não nulo, dado por $v=\sqrt{-\frac{\mu^{2}}{\lambda}}$. Novamente redefinimos o campo $\phi$ como: $\phi^{\prime} \rightarrow \phi-v$ de modo que o VEV do novo campo seja nulo.

Podemos reparametrizar o campo explicitando seu caráter complexo como:

$$
\phi=(v+\eta) e^{i \frac{\zeta}{v}} \approx v+\eta+i \zeta
$$

sendo que a aproximação é válida para pequenas oscilações dos campos $\eta$ e $\zeta$. Colocando essa expressão na lagrangeana e guardando somente os termos de pequenas oscilações obtemos:

$$
\mathcal{L}=\partial_{\mu} \eta \partial^{\mu} \eta+\mu^{2} \eta^{2}+\partial_{\mu} \zeta \partial^{\mu} \zeta-\frac{1}{4} F_{\mu \nu} F^{\mu \nu}+e^{2} v^{2} A_{\mu} A^{\mu}+2 e v \partial_{\mu} \zeta A^{\mu}+\ldots
$$

${ }^{6}$ Para $\mu^{2}>0$ a teoria é somente a QED de dois escalares, $\phi$ e $\phi^{\dagger}$ de massas $\mu$ acoplados com um fóton não massivo. 
o termo cinético do campo $\phi$ dá origem aos termos cinéticos de $\eta$ e $\zeta$, o termo de massa para o fóton e também à interação entre $\zeta$ e o fóton. O potencial $V$ dá origem ao termo de massa para $\eta$ e o termo cinético do fóton fica invariante, o resto da conta representa constantes do tipo $\mu^{2} v^{2}$ que não alteram a lagrangeana. Vemos então que o fóton adquiriu um termo de massa, porém há um termo que o mistura com o $\zeta$. Reescrevendo os últimos termos mais o termo cinético do $\zeta$ podemos ter uma inspiração para uma escolha de gauge conveniente:

$$
e^{2} v^{2}\left(A_{\mu}+\frac{1}{e v} \partial_{\mu} \zeta\right)\left(A^{\mu}+\frac{1}{e v} \partial^{\mu} \zeta\right)
$$

o que sugere uma escolha de gauge do tipo

$$
A_{\mu}^{\prime}=A_{\mu}+\frac{1}{e v} \partial_{\mu} \zeta
$$

Essa escolha corresponde a rotacionar o campo $\phi$ por uma fase $-\zeta$, ou seja: $\phi \rightarrow$ $e^{-i \zeta} \phi=v+\eta$. Com isso concluímos que o campo $\zeta$ não está mais presente na teoria, diz-se que o bóson de gauge "engoliu" o que seria o bóson de Goldstone da teoria. Reescrevendo a lagrangeana ficamos com:

$$
\mathcal{L}=\frac{1}{2}\left[\partial_{\mu} \eta \partial^{\mu} \eta+2 \mu^{2} \eta^{2}\right]-\frac{1}{4} F_{\mu \nu} F^{\mu \nu}+e^{2} v^{2} A_{\mu} A^{\mu}+c
$$

temos então um escalar massivo, $M^{2}=-2 \mu^{2}>0$ e o fóton adquirui uma massa $m_{\gamma}=e v$ e $c$ é uma constante irrelevante. O que seria então o bóson de Goldstone não está mais presente no espectro da teoria. Esse gauge que torna o espectro claro é chamado de gauge unitário. É imediato verificar o número de graus de liberdade, antes da quebra havia 1 escalares complexo ( 2 graus de liberdade) mais um bóson não massivo ( 2 g.d.l). Após a quebra o número permanece o mesmo, pois há somente um escalar real, mas o bóson de gauge adquiriu massa ficando com um grau de liberdade a mais, o longitudinal.

Vimos aqui como o Mecanismo de Higgs faz com que haja uma colaboração entre as teorias de Yang-Mills que eram impedidas de ter um bóson de gauge massivo e teorias com quebra espontânea de uma simetria contínua, que possuiam bósons de gauge não massivos. Quebrando uma simetria local o bóson de gauge adquiriu massa e o bóson de Goldstone não apareceu. Utilizando os conceitos de teorias de gauge e quebra de simetria podemos agora construir o Modelo Padrão. 


\section{Capítulo 3}

\section{O Modelo Padrão}

O Modelo Padrão das Partículas Elementares (MP) é uma teoria baseada em simetrias de calibre que descreve as interações fortes, eletromagnéticas e fracas. Esta secção é uma introdução ao modelo voltada para o leitor que não está familiarizado com o mesmo. Obviamente muitos aspectos de extrema importância para o desenvolvimento do MP, principalmente aspectos experimentais, não poderão ser tratados aqui. Existem inúmeros bons livros que se dedicam parcial ou exclusivamente ao estudo do Modelo Padrão, durante o texto há referências de passagens específicas, mas segue aqui uma lista de referências que são muito mais completas do que o presente texto. Para uma introdução às partículas elementares veja por exemplo [3], [4], [5] e muitos aspectos experimentais em [6], para uma introdução à teoria quântica dos campos e quantização de teorias de calibre [2], [7] e [8]. Para livros e artigos dedicados ao MP veja [1], [9], [10], [11], [12], [13] e [14].

\subsection{Fenomenologia}

Das quatro forças fundamentais que conhecemos na natureza, o Modelo Padrão é uma teoria que descreve três delas: as interações forte, eletromagnética e fraca, sem qualquer preocupação com a gravidade que manifesta seus efeitos somente nas escalas astronômicas.

O conteúdo de matéria no modelo é dividido entre quarks e léptons. Os quarks interagem através de todas as forças, enquanto os léptons não sentem a força forte. Ambos os grupos possuem estrutura igual, são seis constituintes em cada um deles que se agrupam similarmente. Os quarks possuem seis sabores: up, down, charm, strange, top e bottom. Os léptons são: elétron, muon e tau, e seus respectivos neutrinos. Todas as partículas possuem suas correspondentes antipartículas e estão divididas em três famílias

$$
\left(\begin{array}{l}
u \\
d
\end{array}\right),\left(\begin{array}{l}
c \\
s
\end{array}\right),\left(\begin{array}{l}
t \\
b
\end{array}\right)
$$




$$
\left(\begin{array}{c}
e \\
\nu_{e}
\end{array}\right),\left(\begin{array}{c}
\mu \\
\nu_{\mu}
\end{array}\right),\left(\begin{array}{c}
\tau \\
\nu_{\tau}
\end{array}\right)
$$

As propriedades de cada família são idênticas, sendo a massa delas a única característica que as distingue experimentalmente.

Além do conhecimento empírico de que somente os quarks interagem através da força forte, sabe-se também ${ }^{1}$ que as correntes carregadas da força fraca violam paridade maximalmente, de maneira que somente as componentes de mão esquerda das partículas interagem, enquanto as de mão direita são singletos em relação à essa interação.

Deve-se então estudar essas componentes separadamente. Considere a lagrangiana de um férmion sem massa

$$
\mathcal{L}=i \bar{\psi} \not \partial \psi
$$

que dá origem a equação de Dirac para um férmion livre não-massivo

$$
\not \partial \psi=0
$$

Podemos multiplicar essa equação por $\gamma^{5}$ e usar a anticomutação com as matrizes $\gamma^{\mu}$ para ter uma nova solução da equação

$$
\not \partial \gamma^{5} \psi=0
$$

formando combinações lineares dessas duas soluções ainda temos soluções da equação. Podemos então introduzir os projetores $P_{R}$ e $P_{L}$ tais que:

$$
\begin{aligned}
P_{R}^{2} & =P_{R}, \quad P_{L}^{2}=P_{L} \\
P_{R} P_{L} & =P_{L} P_{R}=0
\end{aligned}
$$

e agindo nos espinores $\psi$ temos

$$
\begin{aligned}
& P_{R} \psi \equiv \psi_{R}=\frac{\left(I+\gamma^{5}\right)}{2} \psi \\
& P_{L} \psi \equiv \psi_{L}=\frac{\left(I-\gamma^{5}\right)}{2} \psi
\end{aligned}
$$

Note que podemos escrever $\psi=\psi_{L}+\psi_{R}$, pois $P_{L}+P_{R}=I$. Pode-se então reescrever a lagrangiana (3.1) como

$$
\mathcal{L}=i \bar{\psi}_{L} \not \partial \psi_{L}+i \bar{\psi}_{R} \not \partial \psi_{R}
$$

Caso haja um termo de massa para o férmion, basta acrescentar: $m \bar{\psi} \psi=m\left(\bar{\psi}_{R} \psi_{L}+\bar{\psi}_{L} \psi_{R}\right)$,

\footnotetext{
${ }^{1}$ Através da teoria efetiva de Fermi para as interações fracas.
} 
pois $\bar{\psi}_{R} \psi_{R}=\bar{\psi}_{L} \psi_{L}=0$. Vamos então estudar a lagrangeana do Modelo Padrão por setores para facilitar a didática.

\subsection{Setor de Quebra Espontânea de Simetria}

Como vimos, pedir que uma lagrangeana seja invariante por transformações locais faz com que campos de gauge sejam naturalmente introduzidos na teoria. Introduz-se assim interações entre esses campos bosônicos e os férmions da teoria livre. No MP a simetria local é dada pelo produto de três grupos de gauge.

O grupo $S U(3)_{C}$, onde o índice $C$ remete a cor, é o grupo responsável pelo setor da força forte no MP, tendo $3^{2}-1=8$ bósons de gauge, os chamados glúons. As forças eletromagnética e fraca são descritas pelo modelo de Glashow-Weinberg-Salam. Neste modelo, o grupo de simetria é o produto $S U(2)_{L} \times U(1)_{Y}$, onde o índice $L$ remete a left (esquerda) e $Y$ hipercarga em analogia com a carga elétrica do grupo da QED $U(1)_{E M}$. O número de bósons de gauge na teoria de GWS é $4=\left(2^{2}-1\right)+1$, sendo que através do mecanismo de Higgs, três deles ganham massa e um permanece não massivo.

Portanto o MP é descrito pela simetria de gauge $S U(3)_{C} \times S U(2)_{L} \times U(1)_{Y}$ com a quebra de simetria:

$$
S U(3)_{C} \times S U(2)_{L} \times U(1)_{Y} \rightarrow S U(3)_{C} \times U(1)_{E M}
$$

Para que haja uma quebra espontânea de simetria, vimos que é necessário um campo escalar $\phi$ adquirir um VEV não nulo. Como o setor de cor não sofre alteração, esse campo escalar não interage com os campos de gauge $G^{\mu}$ (que darão origem aos glúons), desse modo $S U(3)$ não é quebrado.

O campo $\phi$ é então um dubleto de $S U(2)_{L}$ e também deve interagir com $U(1)_{Y}$. Para isso é necessário que ele possua uma hipercarga não nula. Introduz-se então o dubleto

$$
\phi \equiv\left(\begin{array}{l}
\phi^{+} \\
\phi^{0}
\end{array}\right)
$$

com hipercarga $Y=+\frac{1}{2}$ e os índices indicam a carga elétrica, A fim de satisfazer a relação de Gell-Mann Nishijima: $Q=T_{3}+Y$, com $Q$ a carga elétrica, $Y$ é a hipercarga e $T_{3}$ é a componente de isospin (aqui $T^{a}=\frac{\sigma^{a}}{2}$ são os geradores de $S U(2)_{L}$, sendo $\sigma$ as matrizes de Pauli) .

O termo escalar da lagrangeana do MP fica então

$$
\mathcal{L}_{\text {escalar }}=D_{\mu} \phi^{\dagger} D^{\mu} \phi-\frac{1}{2} \mu^{2}\left(\phi^{\dagger} \phi\right)-\frac{1}{4} \lambda\left(\phi^{\dagger} \phi\right)^{2}
$$

com $\lambda>0$. Onde identificamos o potencial $V\left(\phi^{\dagger} \phi\right)$ que é o mais geral, renormalizável 
e invariante sob transformaçãoes de $S U(2)$. Com $\mu^{2}<0$ tem-se que $\phi$ adquire um valor esperado de vácuo não nulo

$$
\langle\phi\rangle=\frac{1}{\sqrt{2}}\left(\begin{array}{l}
0 \\
v
\end{array}\right)
$$

com $\left\langle\phi^{\dagger} \phi\right\rangle=-\frac{\mu^{2}}{\lambda}=\frac{v^{2}}{2}$. Com essa escolha, pode-se facilmente ver que nenhum dos geradores $T^{a}$ do grupo $S U(2)$ nem o gerador $Y$ do grupo $U(1)$ deixa o vácuo invariante $(G|0\rangle=0)$, pois:

$$
\begin{aligned}
T^{1}\left(\begin{array}{l}
0 \\
v
\end{array}\right) & =\frac{1}{2}\left(\begin{array}{l}
v \\
0
\end{array}\right) \\
T^{2}\left(\begin{array}{l}
0 \\
v
\end{array}\right) & =\frac{i}{2}\left(\begin{array}{l}
0 \\
v
\end{array}\right) \\
T^{3}\left(\begin{array}{l}
0 \\
v
\end{array}\right) & =-\frac{1}{2}\left(\begin{array}{l}
0 \\
v
\end{array}\right) \\
Y\left(\begin{array}{l}
0 \\
v
\end{array}\right) & =\frac{1}{2}\left(\begin{array}{l}
0 \\
v
\end{array}\right)
\end{aligned}
$$

porém, a combinação linear associada a carga elétrica $Q=T^{3}+Y$ satisfaz $Q|0\rangle=0$. De modo que o gerador do grupo $U(1)_{E M}$ deixa o vácuo invariante e portanto não irá adquirir massa através do Mecanismo de Higgs.

O termo cinético do campo $\phi$ é onde há a interação entre o Higgs e os bósons de Gauge e é responsável pelos termos de massa destes. Como sempre, podemos redefinir o campo $\phi$ como

$$
\phi=e^{i \frac{\sigma}{2} \theta(x)} \frac{1}{\sqrt{2}}\left(\begin{array}{c}
0 \\
v+H(x)
\end{array}\right)
$$

explicitando os 4 graus de liberdade ficam, dos 4 campos reais $\theta^{a}$ e $H$ e devido a invariância sob $S U(2)_{L}$ pode-se escolher a fase local $\alpha^{a}(x)=-\theta^{a}(x)$, de modo que

$$
\phi \rightarrow \phi^{\prime} \equiv e^{-i \frac{\sigma}{2} \theta} \phi=\frac{1}{\sqrt{2}}\left(\begin{array}{c}
0 \\
v+H(x)
\end{array}\right)
$$

este é o chamado Gauge Unitário, onde o espectro de partículas na teoria fica mais claro. Os três campos $\theta^{a}$ seriam os bósons de Goldstone não massivos associados às combinações lineares dos geradores que quebraram a simetria de gauge. Unindo a quebra de simetria com a invariância de gauge, o mecanismo de Higgs é capaz de limpar esses bósons do espectro da teoria, mantendo o mesmo número de graus de liberdade da teoria, como veremos. Adiamos a discussão sobre as interações do Higgs, focando primeiramente nas 
massas das partículas e suas interações.

\subsubsection{Massa dos Bósons EletroFracos}

A derivada covariante para o campo $\phi$ é $D_{\mu} \phi=\left(\partial_{\mu}+i g \tau^{a} W_{\mu}^{a}+i g^{\prime} Y_{\phi} B_{\mu}\right) \phi$, com $W_{\mu}$ e $B_{\mu}$ os bósons de gauge de $S U(2)_{L}$ e $U(1)_{Y}$. Os termos relevantes que darão origem à massa dos bósons de gauge são

$$
\Delta \mathcal{L}=\frac{1}{2}\left(\begin{array}{ll}
0 & v
\end{array}\right)\left(g \tau^{a} W_{\mu}^{a}+g^{\prime} Y_{\phi} B_{\mu}\right)\left(g \tau^{b} W_{\mu}^{b}+g^{\prime} Y_{\phi} B_{\mu}\right)\left(\begin{array}{l}
0 \\
v
\end{array}\right)
$$

avaliando explicitamente os produtos matriciais, $\operatorname{com} \tau=\sigma / 2$ e $Y_{\phi}=1 / 2$, tem-se

$$
\begin{gathered}
\Delta \mathcal{L}=\frac{1}{2}\left(\begin{array}{ll}
0 & v
\end{array}\right)\left(\frac{g^{2} v}{4}\left(\begin{array}{c}
0 \\
\left(W_{\mu}^{1}\right)^{2}+\left(W_{\mu}^{2}\right)^{2}+\left(W_{\mu}^{3}\right)^{2}
\end{array}\right)+\frac{g^{\prime 2} v}{4}\left(\begin{array}{c}
0 \\
\left(B_{\mu}\right)^{2}
\end{array}\right)+\frac{2 g g^{\prime} v}{4}\left(\begin{array}{c}
\left(W_{\mu}^{1}-i W_{\mu}^{2}\right) B^{\mu} \\
-W_{\mu}^{3} B^{\mu}
\end{array}\right)\right) \\
\Rightarrow \Delta \mathcal{L}=\frac{1}{2} \frac{v^{2}}{4}\left[g^{2}\left(\left(W_{\mu}^{1}\right)^{2}+\left(W_{\mu}^{2}\right)^{2}+\left(W_{\mu}^{3}\right)^{2}\right)+g^{\prime 2}\left(B_{\mu}\right)^{2}-2 g g^{\prime} W_{\mu}^{3} B^{\mu}\right] \\
\Rightarrow \Delta \mathcal{L}=\frac{1}{2}\left[\frac{g^{2} v^{2}}{4}\left(\left(W_{\mu}^{1}\right)^{2}+\left(W_{\mu}^{2}\right)^{2}\right)+\frac{v^{2}}{4}\left(-g W_{\mu}^{3}+g^{\prime} B_{\mu}\right)^{2}\right]
\end{gathered}
$$

de onde vemos que dois termos já são diagonais, mas os outros dois campos estão misturados. Diagonalizando esses campos, ou seja rotacionando-os de modo que não haja um termo de mistura, tem-se

$$
\operatorname{det}\left(\begin{array}{cc}
g^{2}-\lambda & g g^{\prime} \\
g g^{\prime} & g^{\prime 2}-\lambda
\end{array}\right)=\lambda^{2}-\lambda\left(g^{2}+g^{\prime 2}\right)=0
$$

ou seja, uma das massas é nula (como esperávamos) e a outra está associada ao auto-valor $\lambda=g^{2}+g^{\prime 2}$. Os autovetores ortogonais correspondentes são:

$$
\frac{1}{\sqrt{g^{2}+g^{\prime 2}}}\left(g^{\prime} W_{\mu}^{3}+g B_{\mu}\right) \quad \frac{1}{\sqrt{g^{2}+g^{\prime 2}}}\left(-g W_{\mu}^{3}+g^{\prime} B_{\mu}\right)
$$

então podemos reescrever os termos de massa da lagrangeana como

$$
\mathcal{L}_{\text {Massa }}=m_{W}^{2} W_{\mu}^{+} W^{-\mu}+m_{Z}^{2} Z_{\mu}^{0} Z^{0 \mu}+m_{\gamma} A_{\mu} A^{\mu}
$$

com

$$
W_{\mu}^{ \pm} \equiv \frac{1}{\sqrt{2}}\left(W_{\mu}^{1} \mp i W_{\mu}^{2}\right) \quad Z_{\mu} \equiv \frac{1}{\sqrt{g^{2}+g^{\prime 2}}}\left(-g W_{\mu}^{3}+g^{\prime} B_{\mu}\right)
$$


sendo

$$
m_{W}=\frac{g v}{2} \quad m_{Z}=\sqrt{g^{2}+g^{\prime 2}} \frac{v}{2}
$$

e a combinação linear associada ao fóton, ortogonal à combinação do $Z_{\mu}^{0}$, continua não massiva

$$
A_{\mu} \equiv \frac{1}{\sqrt{g^{2}+g^{\prime 2}}}\left(g^{\prime} W_{\mu}^{3}+g B_{\mu}\right)
$$

com

$$
m_{\gamma}=0 .
$$

Reescrevendo a derivada covariante em termos dessas combinações lineares tem-se:

$D_{\mu}=\partial_{\mu}+i \frac{g}{\sqrt{2}}\left(W^{+} T^{+}+W^{-} T^{-}\right)+i \frac{1}{\sqrt{g^{2}+g^{\prime 2}}} Z_{\mu}\left(g^{\prime 2} Y-g^{2} T^{3}\right)+i \frac{g g^{\prime}}{\sqrt{g^{2}+g^{\prime 2}}} A_{\mu}\left(T^{3}+Y\right)$

$\operatorname{com} T^{ \pm}=\left(T^{1} \pm i T^{2}\right)$

Como havíamos antecipado o gerador $Q=T^{3}+Y$ não quebrou a simetria e o fóton não adquiriu massa. Identificando o coeficiente deste gerador como a carga elétrica tem-se

$$
e=\frac{g g^{\prime}}{\sqrt{g^{2}+g^{\prime 2}}}
$$

e assim podemos identificar o vértice da $\mathrm{QED} \operatorname{com} Q=-1$ no caso do elétron.

Vale ainda definir mais um parâmetro, o chamado ângulo fraco (Weak) ou de Weinberg, $\theta_{W}$. A redefinição dos campos $W_{\mu}^{3}$ e $B_{\mu}$ foi uma rotação a fim de diagonalizar os termos de massa. O parâmetro da rotação é o ângulo fraco:

$$
\left(\begin{array}{c}
Z^{0} \\
A
\end{array}\right)=\left(\begin{array}{cc}
\cos \theta_{W} & -\sin \theta_{W} \\
\sin \theta_{W} & \cos \theta_{W}
\end{array}\right)\left(\begin{array}{c}
A^{3} \\
B
\end{array}\right)
$$

com isso, identifica-se os parâmetros

$$
\cos \theta_{W}=\frac{g}{\sqrt{g^{2}+g^{\prime 2}}}, \quad \sin \theta_{W}=\frac{g^{\prime}}{\sqrt{g^{2}+g^{\prime 2}}}
$$

portanto

$$
e=g \sin \theta_{W}=g^{\prime} \cos \theta_{W}
$$

Pode-se enfim escrever a derivada covariante em fução dos novos geradores e das redefinições dos parâmetros, de maneira que $\sqrt{g^{2}+g^{\prime 2}}=\frac{g}{\cos \theta_{W}},\left(g^{\prime 2} Y-g^{2} T^{3}\right)=g^{\prime 2} Q-$ $\left(g^{2}+g^{\prime 2}\right) T^{3}$ e ainda $g=\frac{e}{\sin \theta_{W}}$, então:

$$
D_{\mu}=\partial_{\mu}+i \frac{g}{\sqrt{2}}\left(W_{\mu}^{+} T^{+}+W_{\mu}^{-} T^{-}\right)+i \frac{g}{\cos \theta_{W}} Z_{\mu}\left(\sin ^{2} \theta_{W} Q-T^{3}\right)+i e A_{\mu} Q
$$


Rescrevendo as massas dos bósons de gauge, tem-se a relação

$$
m_{Z}=\frac{m_{W}}{\cos \theta_{W}}>m_{W}
$$

que já é uma predição da teoria, indicando que o bóson $Z^{0}$ é mais massivo que os bósons $W^{ \pm}$, fato que é confirmado pela experiência. Com os parâmetros $e, \sin \theta_{W}$ e $m_{Z}$ pode-se calcular todos os efeitos a nível árvore ${ }^{2}$ dos processos com troca dos bósons eletrofracos.

Contando o número de graus de liberdade da teoria, vemos que não houve alteração. Antes da quebra havia 2 campos complexos escalares mais 4 bósons vetoriais não massivos totalizando $2 \times 2+4 \times 2=12$ graus de liberdade. Após a quebra somente um campo escalar real sobrevive (o Higgs) e 3 bósons vetoriais $\left(W^{ \pm}\right.$e $\left.Z^{0}\right)$ adquirem massa, ganhando mais um grau de liberdade longitudinal e 1 deles (o fóton) ainda é não massivo. De modo que tem-se $1 \times 1+3 \times 3+1 \times 2=12$ graus de liberdade!

Através do Mecanismo de Higgs foi possível que os bósons de gauge $W^{ \pm}$e $Z^{0}$ adquirissem massa, porém os férmions da teoria ainda continuam não massivos uma vez que um termo de massa é proibido pela simetria de gauge como veremos na próxima secção.

\subsection{Setor de Férmions}

Antes de discutirmos o termo cinético dos férmions e suas interações com os bósons de gauge vamos ver como eles adquirem massa no MP.

\subsubsection{Massa dos Férmions}

Um termo de massa na Lagrangeana é proibido pela simetria de gauge, pois

$$
\mathcal{L}_{m}=-m \bar{\psi} \psi=-m\left(\bar{\psi}_{L} \psi_{R}+\bar{\psi}_{R} \psi_{L}\right)
$$

mas $\psi_{L}$ é um dubleto de $S U(2)_{L}$ enquanto $\psi_{R}$ é um singleto, de modo que esse termo de massa não é invariante sob $S U(2)_{L} \times U(1)_{Y}$.

Introduzindo uma interação do tipo Yukawa entre os férmions e o campo $\phi$ é possível fazer com que eles ganhem massa. Consideremos primeiramente os léptons: no MP os neutrinos são não massivos, de modo que não há neutrinos de mão direita, uma vez que eles seriam estéreis em relação à qualquer interação. Somente o elétron (e os correspondentes das outras famílias) tem massa. Seja então a interação de Yukawa

$$
\mathcal{L}_{Y}=-y_{e} L_{e} \phi R_{e}+\text { h.c. }
$$

\footnotetext{
${ }^{2}$ Tudo o que foi feito no texto é a descrição clássica da teoria, é então a nível árvore, ou seja, não inclui correções radioativas devido a loops.
} 
aqui $y_{e}$ é uma constante adimensional do acoplamento entre $\phi$ e o férmion, $L_{e}=\left(\begin{array}{c}\nu_{e} \\ e\end{array}\right)_{L}$ é o dubleto de $S U(2)_{L}$ e $R_{e}=e_{R}$ é um singleto de $S U(2)_{L}$ que representa a projeção de mão direita do férmion. Este é somente o termo para o elétron, há uma soma implícita sobre as famílias para gerar massa para o múon e tau. O termo (3.31) é invariante de $S U(2)$ com os índices matriciais de $L$ e $\phi$ somados. Note ainda que essa lagrangeana deve ser invariante de $U(1)_{Y}$ para isso é necessário que $-Y_{L}+Y_{\phi}+Y_{R}=0$ como ficará claro adiante.

Substituindo $\phi$ no gauge unitário tem-se:

$$
\mathcal{L}_{Y}=-y_{e}\left(\begin{array}{ll}
\overline{\nu_{e}} & \bar{e}_{L}
\end{array}\right)\left(\begin{array}{c}
0 \\
\frac{v+H}{\sqrt{2}}
\end{array}\right) e_{R}+\text { h.c. }=-y_{e} \frac{v}{\sqrt{2}} \bar{e}_{L} e_{R}-y_{e} \frac{H(x)}{\sqrt{2}} \bar{e}_{L} e_{R}+\text { h.c. }
$$

onde pode-se ver claramente o termo de massa para o elétron e sua interação com o Higgs, sendo a massa do elétron dada por (3.33).

$$
m_{e}=\frac{y_{e} v}{\sqrt{2}}
$$

Para dar massa aos quarks é necessário introduzir o campo $\widetilde{\phi}$, conjugado de carga do campo $\phi$, tal que $\widetilde{\phi}=i \sigma^{2} \phi$ e $Y_{\widetilde{\phi}}=-Y_{\phi}$. Isso porque para os férmions só era necessário um termo de massa para o elemento com a terceira componente de isospin $-1 / 2$ enquanto que para os quarks também é necessário dar massa ao quarks tipo up cujas componentes de isospin valem $+\frac{1}{2}$. Com isso tem-se então

$$
\begin{gathered}
\mathcal{L}_{Y}=-y_{d}\left(\begin{array}{ll}
\bar{d}_{L} & \bar{u}_{L}
\end{array}\right)\left(\begin{array}{c}
0 \\
\frac{v+H}{\sqrt{2}}
\end{array}\right) d_{R}-y_{u}\left(\begin{array}{ll}
\bar{d}_{L} & \bar{u}_{L}
\end{array}\right)\left(\begin{array}{c}
\frac{v+H}{\sqrt{2}} \\
0
\end{array}\right) u_{R}+h . c \Rightarrow \\
\Rightarrow \mathcal{L}_{Y}=-y_{d} \frac{v}{\sqrt{2}} \bar{d}_{L} d_{R}-\frac{y_{d}}{\sqrt{2}} H \bar{d}_{L} d_{R}-y_{u} \frac{v}{\sqrt{2}} \bar{u}_{L} u_{R}-y_{u} \frac{H}{\sqrt{2}} \bar{u}_{L} u_{R}+\text { h.c. }
\end{gathered}
$$

que também dá origem aos termos de massa e interações com o Higgs, com

$$
m_{q}=\frac{y_{q} v}{\sqrt{2}}
$$

Ainda no setor dos férmions é necessário adicionar na lagrangeana do MP os termos cinéticos desses.

\subsubsection{Termos Cinéticos Fermiônicos}

Para construir o termo cinético dos férmions no Modelo Padrão, ditado por uma simetria local, é necessário introduzir as interações através da derivada covariante. Esta derivada é 
diferente para os quarks e léptons devido às diferentes interações deles e também distingue entre mão direita e mão esquerda, de modo que

$$
\mathcal{L}_{F}=i \bar{q}_{L} \not D q_{L}+i \bar{q}_{R} \not D q_{R}+i \bar{l}_{L} \not D l_{L}+i \bar{l}_{R} \not D l_{R}
$$

com uma soma implícita sobre todos os sabores dos quarks e outra sobre os léptons. Explicitando as derivadas tem-se

$$
\begin{aligned}
i \bar{q}_{L} \not D q_{L} & =\bar{q}_{L} \gamma^{\mu}\left(\partial_{\mu}+i g_{S} \frac{\lambda_{a}}{2} G_{\mu}^{a}+i g \frac{\sigma_{a}}{2} W_{\mu}^{a}+i g^{\prime} Y_{L}^{q} B_{\mu}\right) q_{L} \\
i \bar{q}_{R} \not D q_{R} & =i \bar{q}_{R} \gamma^{\mu}\left(\partial_{\mu}+i g_{S} \frac{\lambda_{a}}{2} G_{\mu}^{a}+i g^{\prime} Y_{R}^{q} B_{\mu}\right) q_{R} \\
i \bar{l}_{L} \not D l_{L} & =\bar{l}_{L} \gamma^{\mu}\left(\partial_{\mu}+i g \frac{\sigma_{a}}{2} W_{\mu}^{a}+i g^{\prime} Y_{L}^{l} B_{\mu}\right) q_{L} \\
i \bar{l}_{R} \not D l_{R} & =\bar{l}_{R} \gamma^{\mu}\left(\partial_{\mu}+i g^{\prime} Y_{R}^{l} B_{\mu}\right) q_{L}
\end{aligned}
$$

sendo $\lambda^{a}$, com $a=1, \ldots, 8$, as matrizes de Gell-Mann geradores do grupo $S U(3)$ e $g_{S}$ a constante de acoplamento forte. As hipercargas $Y$ são tais que a relação $Q=T^{3}+Y$ seja satisfeita. A tabela 3.1 mostra os valores de hipercarga de cada partícula.

\begin{tabular}{|c|c|c|c|c|c|}
\hline Família 1 & Família 2 & Família 3 & $S U(3)_{C}$ & $S U(2)_{L}$ & $U(1)_{Y}$ \\
\hline \hline$\left(\begin{array}{c}u \\
d\end{array}\right)_{L}$ & $\left(\begin{array}{c}c \\
s\end{array}\right)_{L}$ & $\left(\begin{array}{c}t \\
b\end{array}\right)_{L}$ & 3 & 2 & $\frac{1}{6}$ \\
\hline$u_{R}$ & $c_{R}$ & $t_{R}$ & 3 & 1 & $\frac{2}{3}$ \\
\hline$d_{R}$ & $s_{R}$ & $b_{R}$ & 3 & 1 & $-\frac{1}{3}$ \\
\hline$\left(\begin{array}{c}\nu_{e} \\
e\end{array}\right)_{L}$ & $\left(\begin{array}{c}\nu_{\mu} \\
\mu\end{array}\right)_{L}$ & $\left(\begin{array}{c}\nu_{\tau} \\
\tau\end{array}\right)_{L}$ & 1 & 2 & $-\frac{1}{2}$ \\
\hline$e_{R}$ & $\mu_{R}$ & $\tau_{R}$ & 1 & 1 & -1 \\
\hline
\end{tabular}

Tabela 3.1: Números quânticos associados ao grupos $S U(3)_{C}, S U(2)_{L}$ e $U(1)_{Y}$.

\subsubsection{Acoplamentos da QCD}

Os quarks são tripletos de cor, então deve haver uma soma sobre as cores dos campos dos quarks. Explicitando a interação forte no termo cinético da lagrangeana dos quarks tem-se:

$$
\mathcal{L}_{F}^{Q C D}=\sum_{f}\left(i \bar{q}_{f} \not q_{f}+i g_{S} G_{\mu}^{a} \bar{q}_{f}^{\alpha} \gamma^{\mu} \frac{\lambda_{\alpha \beta}^{a}}{2} q_{f}^{\beta}\right)
$$

sendo $q_{f}^{\alpha}$ um quark de sabor $f$ e cor $\alpha$. Para obtermos a lagrangeana completa da $Q C D$ ainda resta obter a interação entre os glúons que aparece devido ao termo cinético desses que apresentamos na secção 3.4.

Vamos agora analisar a interação dos férmions com os bósons eletrofracos. Por simplicidade considera-se apenas uma família, mas a extensão é imediata. 


\subsubsection{Acoplamentos com os Bósons de Gauge EletroFracos}

Utilizando as combinações lineares definidas em (3.19) e (3.21) para os bósons $W^{ \pm}, Z^{0}$ e

$\gamma$, pode-se escrever a lagrangeana dos férmions $(3.36)$ como $^{3}$

$$
\begin{aligned}
\mathcal{L}_{F}= & \bar{L} i \not \partial L+\bar{e}_{R} i \not \partial e_{R}+\bar{Q}_{L} i \not \partial Q_{L}+\bar{u}_{R} i \not \partial u_{R}+\bar{d}_{R} i \not \partial d_{R}+ \\
& -g\left(W_{\mu}^{+} J_{W}^{\mu+}+W_{\mu}^{-} J_{W}^{\mu-}+Z_{\mu} J_{Z}^{\mu}\right)-e A_{\mu} J_{E M}^{\mu}
\end{aligned}
$$

sendo $L \equiv\left(\begin{array}{c}\nu_{e} \\ e\end{array}\right)_{L}, Q_{L} \equiv\left(\begin{array}{l}u \\ d\end{array}\right)_{L}$ e ainda

$$
\begin{gathered}
J_{W}^{\mu+}=\frac{1}{\sqrt{2}}\left(\bar{\nu}_{L} \gamma^{\mu} e_{L}+\bar{u}_{L} \gamma^{\mu} d_{L}\right) \\
J_{W}^{\mu-}=\frac{1}{\sqrt{2}}\left(\bar{e}_{L} \gamma^{\mu} \nu_{L}+\bar{d}_{L} \gamma^{\mu} u_{L}\right) \\
J_{Z}^{\mu}=\frac{1}{\cos \theta_{W}}\left[\bar{\nu}_{L} \gamma^{\mu}\left(\frac{1}{2}\right) \nu_{L}+\bar{e}_{L} \gamma^{\mu}\left(\frac{1}{2}-\sin ^{2} \theta_{W}\right) e_{L}+\bar{e}_{R} \gamma^{\mu}\left(-\sin ^{2} \theta_{W}\right) e_{R}+\right. \\
+\bar{u}_{L} \gamma^{\mu}\left(-\frac{1}{2}+\frac{2}{3} \sin ^{2} \theta_{W}\right) u_{L}+\bar{u}_{R} \gamma^{\mu}\left(\frac{2}{3} \sin ^{2} \theta_{W}\right) u_{R}+ \\
\left.+\bar{d}_{L} \gamma^{\mu}\left(\frac{1}{2}-\frac{1}{3} \sin ^{2} \theta_{W}\right) d_{L}+\bar{d}_{R} \gamma^{\mu}\left(-\frac{1}{3} \sin ^{2} \theta_{W}\right) d_{R}\right] \mathrm{e} \\
J_{E M}^{\mu}=\bar{e} \gamma^{\mu}(-1) e+\bar{u} \gamma^{\mu}\left(+\frac{2}{3}\right) u+\bar{d} \gamma^{\mu}\left(-\frac{1}{3}\right) d .
\end{gathered}
$$

Analisando a corrente $J_{E M}^{\mu}$, observa-se a bem conhecida corrente eletromagnética da QED. Percebe-se claramente que não há mistura entre as componentes do dubleto e não há violação de paridade, pois as componentes esquerda e direita se juntam formando $u=u_{L}+u_{R}$. Vale também notar o caráter vetorial/axial das correntes carregadas, conhecidos da teoria de Fermi como vértices $V-A$. Pode-se escrever

$$
\bar{\nu}_{L} \gamma^{\mu} e_{L}=\bar{\nu} \frac{\left(I+\gamma^{5}\right)}{2} \gamma^{\mu} \frac{\left(I-\gamma^{5}\right)}{2} e=\bar{\nu} \gamma^{\mu} \frac{\left(I-\gamma^{5}\right)^{2}}{4} e=\bar{\nu} \gamma^{\mu} \frac{\left(I-\gamma^{5}\right)}{2} e
$$

fazendo com que a paridade seja violada maximalmente nas correntes carregadas.

Para a corrente neutra do $Z^{0}$ o caráter vetorial/axial aparece se usarmos $f_{L}=P_{L} f$ e $f_{R}=P_{R} f_{R}$ sendo $f$ um férmion $e, \nu, u$ ou $d$. Definindo os parâmetros $g_{V}$ e $g_{A}$ pode-se reescrever $J_{Z}^{\mu}$ como

$$
J_{Z}^{\mu}=\frac{1}{2 \cos \theta_{W}} \bar{f} \gamma^{\mu}\left[g_{V}^{f}-g_{A}^{f} \gamma^{5}\right] f
$$

\footnotetext{
${ }^{3}$ Lembrando que para clareza de notação utilizamos somente uma família.
} 
com

$$
\begin{gathered}
g_{V}^{f}=2 Q_{f} \sin ^{2} \theta_{W}-t_{f}^{3} \\
g_{A}^{f}=-t_{f}^{3}
\end{gathered}
$$

sendo $Q_{f}$ a carga do férmion, que vale -1 para o caso do elétron, e $t_{f}^{3}$ é a componente de isospin, no caso do elétron $t_{e}^{3}=-\frac{1}{2}$. A tabela 3.2 mostra os valores para todos os férmions.

\begin{tabular}{|c|c|c|}
\hline Férmions & $g_{V}^{f}$ & $g_{A}^{f}$ \\
\hline \hline quarks $u p$ & $\frac{4}{3} \sin ^{2} \theta_{W}-\frac{1}{2}$ & $-\frac{1}{2}$ \\
\hline quarks down & $-\frac{2}{3} \sin ^{2} \theta_{W}+\frac{1}{2}$ & $+\frac{1}{2}$ \\
\hline neutrinos & $-\frac{1}{2}$ & $-\frac{1}{2}$ \\
\hline léptons carregados & $-2 \sin ^{2} \theta_{W}+\frac{1}{2}$ & $+\frac{1}{2}$ \\
\hline
\end{tabular}

Tabela 3.2: Valores de $g_{V}$ e $g_{A}$ para os férmions.

\subsection{Setor de Gauge}

Para completar a lagrangeana do MP é necessário acrescentar os termos cinéticos dos bósons de gauge. Como vimos na secção sobre teorias de gauge não-abelianas, os termos cinéticos são do tipo

$$
\begin{aligned}
\mathcal{L}_{\text {gauge }}= & -\frac{1}{4} B^{\mu \nu} B_{\mu \nu}-\frac{1}{2} \operatorname{Tr}\left(\frac{\sigma^{a}}{2} W_{\mu \nu}^{a} \frac{\sigma^{b}}{2} W^{b \mu \nu}\right)-\frac{1}{2} \operatorname{Tr}\left(\frac{\lambda^{a}}{2} G_{\mu \nu}^{a} \frac{\lambda^{b}}{2} G^{b \mu \nu}\right)= \\
= & -\frac{1}{4} B^{\mu \nu} B_{\mu \nu}-\frac{1}{4} W_{\mu \nu}^{a} W_{a}^{\mu \nu}-\frac{1}{4} G_{\mu \nu}^{a} G_{a}^{\mu \nu}
\end{aligned}
$$

e devido à invariância de calibre, termos de massa para esses bósons são proibidos, mas o Mecanismo de Higgs gera massa para três combinações lineares destes bósons.

Devido ao caráter não-abeliano do grupo $S U(3)$ os glúons interagem entre si. O mesmo acontece entre os bósons $W^{ \pm}, Z^{0}$ e o fóton $\gamma$. Vamos ver aqui a interação entre os bósons eletrofracos, sabendo que a interação entre os glúons é análoga e segue o mesmo raciocínio.

\subsubsection{Interação entre os bósons de gauge eletrofracos}

Expandindo o termo cinético dos bósons de $S U(2)$ tem-se:

$$
\mathcal{L}_{W}=-\frac{1}{4} W_{\mu \nu}^{a} W_{a}^{\mu \nu}=\mathcal{L}_{0 W}-g f^{a b c}\left(\partial_{\mu} W_{\nu}^{a}\right) W_{b}^{\mu} W_{c}^{\nu}-\frac{1}{4} g^{2}\left(f^{e a b} W_{\mu}^{a} W_{\nu}^{b}\right)\left(f^{e c d} W_{c}^{\mu} W_{d}^{\nu}\right)
$$


onde $\mathcal{L}_{0 W}$ representa os termos cinéticos usuais e vê-se que os outros dois são acoplamentos cúbicos e quárticos dos bósons que só estão presentes devido a não comutação entre os campos $W^{a}$.

Reescrevendo os termos em (3.37) em função dos campos $W_{\mu}^{ \pm}, Z_{\mu}^{0}$ e $A_{\mu}$ pode-se ver a interação entre eles:

$$
\begin{aligned}
\mathcal{L}_{W W \gamma} & =i e\left[\left(\partial^{\mu} W^{-\nu}-\partial^{\nu} W^{-\mu}\right) W_{\mu}^{+} A_{\nu}-\left(\partial^{\mu} W^{+\nu}-\partial^{\nu} W^{+\mu}\right) W_{\mu}^{-} A_{\nu}\right] \\
\mathcal{L}_{W W Z} & =i e \cot \theta_{W}\left[\left(\partial^{\mu} W^{-\nu}-\partial^{\nu} W^{-\mu}\right) W_{\mu}^{+} Z_{\nu}-\left(\partial^{\mu} W^{+\nu}-\partial^{\nu} W^{+\mu}\right) W_{\mu}^{-} Z_{\nu}\right] \\
\mathcal{L}_{W W W W} & =-\frac{e^{2}}{2 \sin ^{2} \theta_{W}}\left[\left(W_{\mu}^{+} W^{-\mu}\right)^{2}-W_{\mu}^{+} W^{+\mu} W_{\nu}^{-} W^{-\nu}\right] \\
\mathcal{L}_{W W Z Z} & =-e^{2} \cot ^{2} \theta_{W}\left[W_{\mu}^{+} W^{-\mu} Z_{\nu} Z^{\nu}-W \mu^{+} Z^{\mu} W_{\nu}^{-} Z^{\nu}\right] \\
\mathcal{L}_{W W \gamma \gamma} & =-e^{2}\left[W_{\mu}^{+} W^{-\mu} A_{\nu} A^{\nu}-W_{\mu}^{+} A^{\mu} W^{-\nu} A_{\nu}\right] \\
\mathcal{L}_{W W Z \gamma} & =-e^{2} \cot \theta_{W}\left[2 W_{\mu}^{+} W^{-\mu} Z_{\nu} A^{\nu}-W_{\mu}^{+} Z^{\mu} W^{-\nu} A_{\nu}-W_{\mu}^{+} A^{\mu} W_{\nu}^{-} Z^{\nu}\right]
\end{aligned}
$$

estes termos dão origem aos diagramas de Feynman da figura 3.1.
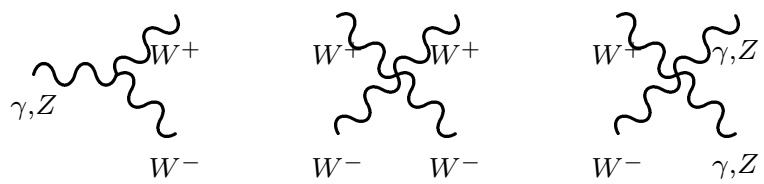

Figura 3.1: Vértices de interações entre os bósons de gauge eletrofracos.

Para os glúons o resultado é análogo, mas não há interações desses com os outros bósons do MP e as constantes de estrutura do grupo não são os tensores antissimétricos $\epsilon^{a b c}$ usados na computação de $S U(2)$. Sendo $f^{a b c}$ as constantes de estrutura de $S U(3)$ tem-se:

$$
\mathcal{L}_{Q C D}^{\text {gauge }}=\frac{g_{S}}{2} f^{a b c}\left(\partial_{\mu} G_{\nu}^{a}-\partial_{\nu} G_{\mu}^{a}\right) G^{b \mu} G^{c \nu}-\frac{g_{S}^{2}}{4} f^{a b c} f_{a d e} G_{b}^{\mu} G_{c}^{\nu} G_{\mu}^{d} G_{\nu}^{e}
$$

que dão origem aos diagramas da figura 3.2.
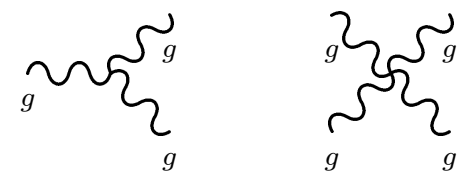

Figura 3.2: Auto-interações cúbica e quártica dos glúons.

\subsection{Autoestados de Massa, Autoestados de Gauge e a Matriz CKM}

Quando escrevemos a interação dos férmions com os bósons eletrofracos (3.39) na secção 3.3.4 utilizamos somente uma família. Porém devido ao mecanismo de geração de massa, 
termos que misturam famílias aparecem na lagrangeana. Considere então o vetor $u_{L}^{i}$ que representa todos os quarks tipo up e o vetor $d_{L}^{i}$ que representa os tipo down. Assim

$$
u_{L}^{i}=\left(u_{L}, c_{L}, t_{L}\right), \quad d_{L}^{i}=\left(d_{L}, s_{L}, b_{L}\right)
$$

são vetores na base de massa, ou seja, são os autoestados da matriz que diagonaliza os termos de massa. De maneira que a mistura ocorrerá no setor de gauge e o setor de Yukawa fica diagonal ${ }^{4}$. Na base que diagonaliza o setor de interação com os bósons de gauge tem-se $u_{L}^{i}$ e $d_{L}^{i}$. Os vetores se relacionam através das transformações unitárias $U_{u}$ e $U_{d}$ da seguinta maneira:

$$
u_{L}^{i}=U_{u}^{i j} u_{L}^{\prime j}, \quad d_{L}^{i}=U_{d}^{i j} d_{L}^{\prime j}
$$

podemos então reescrever as interações das correntes (3.39) nesta nova base. É fácil notar que as correntes neutras do $Z^{0}$ e da QED não sofrerão nenhuma mudança. Isso porque só há interações entre os mesmos elementos de um dubleto e as matrizes de transformação se cancelam, por exemplo

$$
\bar{u} \gamma^{\mu} u=\bar{u}^{\prime} \gamma^{\mu} U_{u}^{\dagger} U_{u} u^{\prime}=\bar{u}^{\prime} \gamma^{\mu} u^{\prime}
$$

o mesmo acontece nos termos da corrente do $Z^{0}$, pois há também uma matriz de transformação para as componentes de mão direita, mas essas se cancelam de modo análogo. Então $J_{E M}$ e $J_{Z}$ não sofrem alterações (mecanismo de GIM [15]). Então as correntes neutras do MP não alteram sua forma se escritas na base de massa, consequência direta de tratarmos igualmente todos os férmions com mesma carga elétrica. Vale notar ainda que os neutrinos não interagem com o bóson de Higgs, pois não introduzimos um termo de Yukawa com $\widetilde{\phi}$ para dar massa a eles. Desse fato podemos ver que não há mistura no setor leptônico da teoria, mesmo nas correntes carregadas. Isso porque podemos escolher o vetor $\nu_{i}$ convenientemente, uma vez que ele só aparece no setor de gauge, a fim de cancelar as matrizes de transformação de base.

O evento de interesse nessa discusão ocorre nas correntes carregadas. São esses termos que misturam famílias e portanto as matrizes de transformação não se cancelam, uma vez que são diferentes $U_{u}^{\dagger} U_{d} \neq I$. Tomemos como exemplo a corrente positiva

$$
J_{W}^{\mu+}=\bar{u}_{L} \gamma^{\mu} d_{L}+\ldots=\bar{u}_{L}^{\prime i} \gamma^{\mu}\left(U_{u}^{\dagger}\right)_{i k}\left(U_{d}\right)_{k j} d_{L}^{j}+\ldots
$$

define-se assim a matriz $V_{i j}=\left(U_{u}^{\dagger} U_{d}\right)_{i j}$ denominada matriz $C K M$, ou Cabibbo-KobayashiMaskawa. Os termos não diagonais desta matriz dão origem às misturas dos sabores de quark, podendo haver transições de um quark tipo up para qualquer um do tipo down.

\footnotetext{
${ }^{4}$ Pois os estados físicos são definidos pelos autovetores da matriz de massa.
} 
Os termos dessa matriz são dados por:

$$
V \equiv\left(\begin{array}{ccc}
V_{u d} & V_{u s} & V_{u b} \\
V_{c d} & V_{c s} & V_{c b} \\
V_{t d} & V_{t s} & V_{t b}
\end{array}\right)=\left(\begin{array}{ccc}
0.97428 \pm 0.00015 & 0.2253 \pm 0.0007 & 0.00347_{-0.00012}^{+0.00016} \\
0.2252 \pm 0.0007 & 0.97345_{-0.00016}^{+0.00015} & 0.0410_{-0.0007}^{+0.0011} \\
0.00862_{-0.00020}^{+0.00026} & 0.0403_{-0.0007}^{+0.0011} & 0.999152_{-0.000045}^{+0.000030}
\end{array}\right)
$$

sendo que os valores numéricos são experimentais. Observa-se que os termos diagonais de $V$ são próximos de 1 enquanto os não diagonais são suprimidos, ou seja, as misturas de gerações são suprimidas, mas existem.

No caso de $N$ gerações de quarks, existem $2 N$ sabores de modo que os parâmetros de entrada na matriz são:

- Uma matriz $N \times N$ (que satisfaz $V^{\dagger} V=I$ ) necessita de $N^{2}$ parâmetros;

- Destes $N^{2}$ parâmetros, $2 N-1$ não são importantes fisicamente, pois se tratam de fases que podem ser absorvidas redefinindo os campos dos quarks, seja na base de massa ou de interação, tem-se então $N^{2}-(2 N-1)=(N-1)^{2}$ parâmetros;

- $\frac{N(N-1)}{2}$ são ângulos de rotação, chamados de ângulos de mistura;

- Os $\frac{(N-1)(N-2)}{2}$ restantes são fases complexas que causam violação de $C P$.

Historicamente, Cabibbo considerou somente duas gerações de quark, de maneira que só havia um parâmetro na matriz, um ângulo de mistura conhecido como ângulo de Cabibbo, $\theta_{C}$. No MP temos $N=3$ e então, existem três ângulos de mistura e uma fase complexa, fonte de toda violação de $C P$ do Modelo Padrão. Kobayashi e Maskawa previram a existência de uma terceira geração observando que a violação de $C P$ de sistemas de kaons neutros era impossível com somente duas famílias.

Como exemplo da teoria de Cabibbo, considere as duas primeiras gerações. Temos que

$$
V_{1 j} d_{L}^{j}=\cos \theta_{C} d_{L}^{\prime}+\sin \theta_{C} s_{L}^{\prime}
$$

onde nota-se que o decaimento de um quark $s$ em um quark $u$ é permitido, porém há uma supressão de Cabibbo devido ao coeficiente $\sin \theta_{C}<\cos \theta_{C}$.

\subsection{Setor de Higgs}

Substituindo o campo escalar $\phi$, introduzido na lagrangeana para quebra de simetria, no gauge unitário podemos escrever os termos que dependem do campo de Higgs $H$.

$$
\mathcal{L}_{\text {Higgs }}=\mathcal{L}_{\text {escalar }}+\mathcal{L}_{Y}=\mathcal{L}_{K}+\mathcal{L}_{V}+\mathcal{L}_{Y}
$$


onde separamos o setor de Higgs na lagrangeana num termo cinético, potencial e na interação de Yukawa. O termo cinético dá origem às interações com os bósons de gauge, presentes na derivada covariante. O potencial gera auto-interações cúbicas e quárticas do bóson de Higgs e a interação tipo Yukawa é responsável pelo acoplamento do Higgs com os férmions.

Fazendo $v \rightarrow v+H$ nos termos de massa dos férmions, vê-se que a interação entre o Higgs e os férmions é análoga à interação fotônica, sem violação de carga e sabor como se espera, dando origem à diagramas como o da figura 3.3. O acoplamentoé dado por: $\frac{m_{f}}{v}$, ou seja, todos os acoplamentos são proporcionais à massa do férmion e do VEV, de maneira que a razão de ramificação do Higgs é maior para os férmions mais pesados ${ }^{5}$.

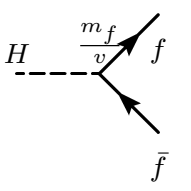

Figura 3.3: Acoplamento entre os férmions e o bóson de Higgs.

Procedendo da mesma forma nos termos de massa dos bósons de Gauge, temos interações com dois $W^{\prime} s$ e um ou dois Higgs, devido à dependência quadrática do VEV com os bósons

$$
\frac{g^{2} v^{2}}{2} W_{\mu}^{+} W^{\mu-} \rightarrow \frac{g^{2}(v+H)^{2}}{2} W_{\mu}^{+} W^{\mu-} \propto \frac{g v H}{2} W_{\mu}^{+} W^{\mu-}+\frac{g^{2} H^{2}}{4} W_{\mu}^{+} W^{\mu-}
$$

dando origem às interações da figura 3.4.
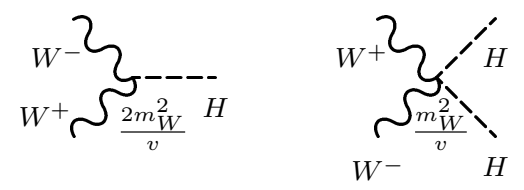

Figura 3.4: Interações entre o Higgs e o W.

E analogamente para o $Z^{0}$ com os diagramas da figura 3.5.
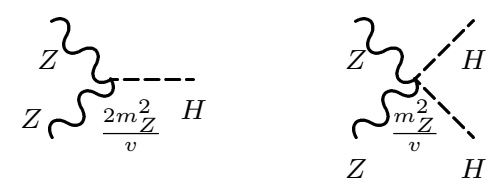

Figura 3.5: Interações entre o Higgs e o Z.

Aqui também os acoplamentos dependem da massa dos bósons e do VEV, mas a dependência na massa é quadrática.

\footnotetext{
${ }^{5} \mathrm{O}$ decaimento deve ser cinematicamente possível, para $M_{H}<2 m_{t}$, o decaimento $H \rightarrow t \bar{t}$ é proibido cinematicamente.
} 
Desta lagrangeana ainda pode-se ver as auto interações do Higgs e seu termo de massa, provenientes do potencial.

$$
\mathcal{L}_{V}=-\frac{1}{2} M_{H}^{2} H^{2}-\frac{M_{H}^{2}}{2 v} H^{3}-\frac{M_{H}^{2}}{8 v^{2}} H^{4}
$$

com

$$
M_{H}=\sqrt{2 \lambda} v=\sqrt{-2 \mu^{2}}
$$

como o parâmetro $\lambda$ é renormalizável e desconhecido, não sabemos da teoria a massa do Higgs, sendo um parâmetro que precisa ser medido experimentalmente, assim como as massas dos férmions por não conhecermos os acoplamentos de Yukawa. Mas as correções à massa do Higgs geram alguns problemas como veremos mais adiante. Seja qual for a solução adotada para solucionar esse problema, o novo modelo deve ser capaz de reproduzir a quebra da simetria $S U(2)_{L} \times U(1)_{Y}$.

Relações como $m_{W}=m_{Z} \cos \theta_{W}$ são medidas com precisão de $1 \%$ e levam em consideração correções de 1-loop. De modo que qualquer outro mecanismo de quebra de simetria diferente do MP que se escolha deve ser capaz de reproduzir todos os sucessos do Modelo Padrão que passamos a descrever.

\subsection{Testes do Modelo Padrão}

O Modelo Padrão é uma teoria com alto poder preditivo, apesar de conter um grande número de parâmetros livres (presentes principalmente no setor de Yukawa e o setor de Higgs). No setor de interações entre os férmions e os bósons de Gauge foi possível realizar inúmeros testes experimentais e até agora o MP passou em todos os testes realizados neste setor, mas inúmeras questões ainda permanecem abertas. Nesta secção comentamos somente alguns desses testes para ilustrar o sucesso do modelo, mas para o leitor mais interessado recomenda-se a leitura de artigos e livros dedicados a esses testes como os citados no início da secção 3 especialmente os livros [6] e [10] com capítulos voltados às descobertas experimentais.

Mostra-se abaixo apenas três exemplos de processos decorrentes das interações de gauge que puderam ser observados nos experimentos com aceleradores. Porém o MP possui muito mais predições do que as apresentadas aqui. No final desta secção resumese esse sucesso com alguns resultados do LEP que foi um dos aceleradores que mais contribuiram para as medidas de precisão que temos hoje.

\subsubsection{A Massa dos Bósons de Gauge Eletrofracos}

A partir de experimentos com o decaimento do muon, $\mu^{-} \rightarrow e^{-} \tilde{\nu}_{e} \nu_{\mu}$ pode-se obter a medida mais precisa na medição da constante de Fermi, $G_{F}=1.16635(1) \cdot 10^{-5} \mathrm{GeV}^{-2}$. 
Calculando a amplitude para esse decaimento usando o MP temos:

$$
\mathcal{M}=\frac{g^{2}}{2} \bar{\nu}_{\mu L} \gamma^{\alpha} \mu_{L} \frac{(-i) g_{\alpha \beta}}{q^{2}-m_{W}^{2}} \bar{\nu}_{e L} \gamma^{\beta} e_{L}
$$

no limite efetivo de baixas energias, $q^{2} \ll M_{W}^{2}$ tem-se a teoria de Fermi, de modo que pode-se aproximar este diagrama pela interação de quatro férmions e identificar

$$
\frac{G_{F}}{\sqrt{2}}=\frac{g^{2}}{8 m_{W}^{2}}
$$

e como $m_{W}=\frac{g v}{2}$ conclui-se que

$$
G_{F}=\frac{1}{\sqrt{2} v^{2}} \Rightarrow v \approx 246 \mathrm{GeV}
$$

e como vimos anteriormente, podemos tratar todos os processos que envolvem as correntes fracas usando os parâmetros, e, $\theta_{W}$ e $m_{Z}$. Se quisermos tratar processos com o Higgs é necessário acrescentar sua massa $M_{H}$ como parâmetro. Usando as relações desses parâmetros e suas definições podemos trocá-los por $G_{F}, \alpha^{-1}, m_{Z}$ e $M_{H}$. Essa troca tem a vantagem de usar os mais bem conhecidos parâmetros medidos experimentalmente.

Em nível árvore obtemos $\sin ^{2} \theta_{W} \approx 0.212$ e usando a razão das massas dos bósons de gauge, temos $m_{W} \approx 80.94 \mathrm{GeV}$, em grande concordância com o valor medido. Usando ainda correções a 1-loop a concordância com o valor experimental é ainda maior como mostraremos mais adiante.

\subsubsection{Assimetria na Corrente Neutra Fraca}

Um outro teste do Modelo é através das correntes neutras. A lagrangeana do MP trata diferentemente os férmions de mão direita e mão esquerda de maneira que podemos definir uma assimetria de polarização. No decaimento $Z^{0} \rightarrow f \bar{f}$, por exemplo, espera-se comportamentos distintos para quiralidades distintas de maneira que

$$
A_{L R}^{f} \equiv \frac{\Gamma\left(Z^{0} \rightarrow f_{L} \bar{f}_{R}\right)-\Gamma\left(Z^{0} \rightarrow f_{R} \bar{f}_{L}\right)}{\Gamma\left(Z^{0} \rightarrow f_{L} \bar{f}_{R}\right)+\Gamma\left(Z^{0} \rightarrow f_{R} \bar{f}_{L}\right)}
$$

seja não nulo. Calculando explicitamente temos

$$
A_{L R}^{f}=\frac{\left(\frac{1}{2}-2\left|Q_{f}\right| \sin ^{2} \theta_{W}\right)^{2}-\left(2\left|Q_{f}\right| \sin ^{2} \theta_{W}\right)^{2}}{\left(\frac{1}{2}-2\left|Q_{f}\right| \sin ^{2} \theta_{W}\right)^{2}+\left(2\left|Q_{f}\right| \sin ^{2} \theta_{W}\right)^{2}}
$$

mostrando claramente a assimetria. Usando $\sin ^{2} \theta_{W} \approx 0.23$ tem-se $A=15 \%$ para os léptons carregados e $95 \%$ para os quarks $d, s$ e $b$. Além desta assimetria, existem outras que são causadas pelas correntes fracas, veja por exemplo [13]. 


\subsubsection{Violação de CP}

Mais um teste que vale a pena comentar é a violação de CP em sistemas de hádrons. Vimos no capítulo 2 alguns conceitos de simetria e três das mais importantes simetrias discretas da física são conhecidas como: conjugação de carga, C; inversão de paridade, P; e inversão temporal, T.

O teorema CPT garante que para uma teoria quântica de campo, com invariância de Lorentz e que preserva micro-causalidade, o produto dessas três simetrias é invariante. Então qualquer sistema físico do MP deve ser invariante sob transformações CPT, de maneira que uma violação de CP implica necessariamente numa violação oposta de $\mathrm{T}$, a fim de preservar a invariância.

Muitos processos físicos são invariantes por CP (e consequentemente T), ou seja, invariantes na troca, férmion de mão esquerda $\Leftrightarrow$ anti-férmion de mão direita. Um exemplo de violação de paridade foi visto nas interações fracas, as correntes carregadas em particular violam a paridade maximalmente, pois só se acoplam com uma das quiralidades. Mas violações de CP são mais raras na natureza, ainda assim, é possível observá-las.

Violações de CP da ordem de $0.2 \%$ foram observadas em sistemas de kaons neutros e mais recentemente nas fábricas de $B^{\prime} s$ em níveis maiores. Vimos na discussão da matriz CKM na secção 3.5, que a única fonte capaz de produzir violação de CP no MP é a fase complexa presente na matriz de mistura dos quarks. Isso impõe vínculos nas predições do MP, pois toda violação observada deve provir dessa fase. Vimos que somente com duas gerações de férmions não seria possível explicar essa violação observada, pois não há uma fase complexa, apenas um ângulo de mistura. No sistema de kaons, por exemplo, a violação só aparece através de correções de 1-loop envolvendo o quark top.

Além disso, todos os elementos da matriz CKM devem ser não nulos e todos os quarks devem possuir massas diferentes, sem que haja degenerescência na massa. Se qualquer um desses requisitos não acontecesse no MP, essa fase complexa poderia ser redefinida por uma rotação nos campos dos quarks e sumiria, fazendo com que não houvesse uma fonte de violação de $\mathrm{CP}$.

É possível mostrar [13] que o tamanho da violação é proporcional ao produto dos ângulos de mistura, de maneira que em geral, são efeitos pequenos. Além disso, mostrase também que esses efeitos somem para altas energias, quando a massa dos quarks é praticamente degenerada. Eventos que envolvem os Mésons B são muito importantes no estudo da simetria CP, pois envolvem processos em que há violação já a nível árvore, sem a necessidade de correções.

Diversos experimentos mostram uma violação compatível com previsões teóricas. É interessante notar que nesses experimentos a violação de inversão temporal (T) também ocorre e é exatamente o necessário para que CPT seja uma boa simetria da natureza!

A violação de $\mathrm{CP}$ é essencial no Universo em que vivemos para explicar a assimetria 
que existe entre matéria e anti-matéria no Universo. Porém somente o Modelo Padrão não é suficiente para explicar essa assimetria.

\subsubsection{Considerações Experimentais}

Em resumo, sabe-se que o MP passou por inúmeros testes experimentais, comprovando seu alto poder preditivo. Um forte indício desse poder foi através das correções radioativas do modelo que puderam ser testadas com precisão de até $0.1 \%$. Com a construção de diversos aceleradores como o LEP e o Tevatron, os processos tiveram de ser calculados com mais ordens de perturbação, devido a grande precisão dos experimentos. O sucesso do MP se mostrou justamente nesses cálculos de correções da QED e QCD. Conforme os testes de precisão foram sendo feitos, as predições sempre se mostraram compatíveis com o valor experimental. Abaixo, na figura 3.6, mostramos uma comparação entre os dados experimentais de diversos testes e o melhor ajuste do MP.

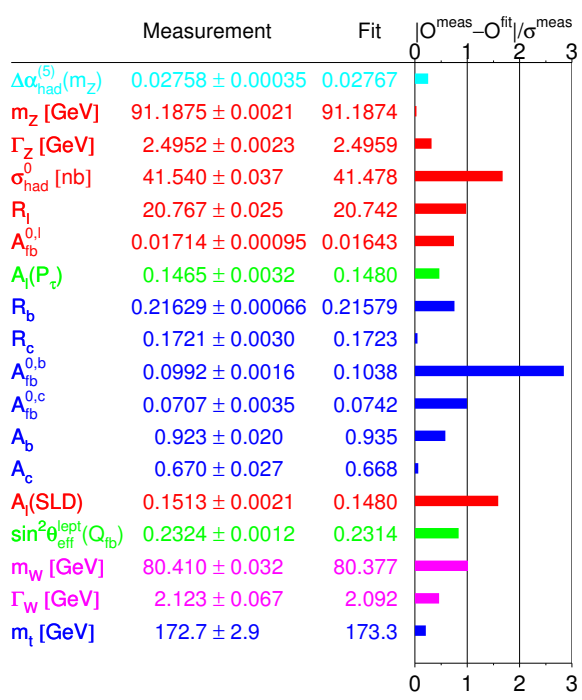

Figura 3.6: Poder preditivo do MP conforme medições do LEP, [23].

Observa-se que todas as previsões mostradas na figura estão de acordo com as medições dentro de até $3 \sigma$ de erro.

Inúmeros outros testes foram realizados, principalmente no setor eletrofraco da teoria, medindo os acoplamentos entre os bósons de gauge e entre esses e os férmions. As massas e larguras de decaimento dos bósons $W^{ \pm}$e $Z^{0}$, também foram testadas exaustivamente no Tevatron e LEP, nas conhecidas fábricas de $Z^{\prime} s$ (devido a energia do centro de massa da colisão ser muito próxima da massa do $Z^{0}$ ). O número de famílias e gerações também se mostram em grande acordo com a experiência, o decaimento invisível do $Z^{0}$ em neutrinos 
pôde determinar o número de neutrinos leves como sendo compatível com 3. A universalidade do acoplamento fraco e eletromagnético e a mistura dos quarks e a matrix CKM vista na secção 3.5 também são testes bem sucedidos do MP. Há ainda muitos outros que deixamos de citar aqui.

A figura 3.7, por exemplo, mostra os acoplamentos vetorial e axial dos léptons, conforme definidos na seç̧ão 3.3.4.

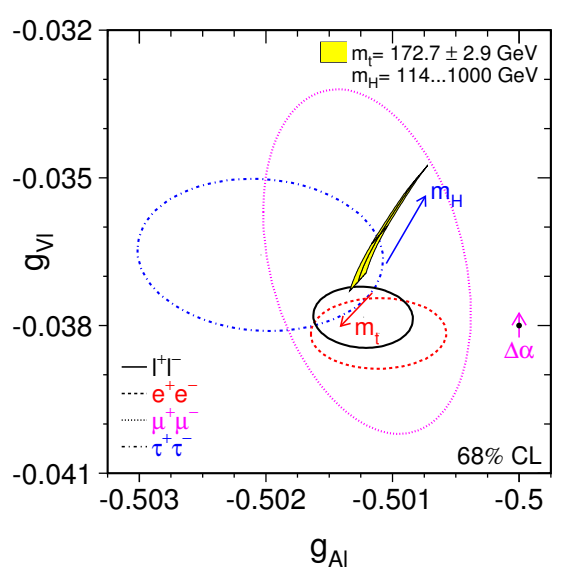

Figura 3.7: Acoplamentos leptônicos vetorial e axial, com base nos dados do LEP, [23].

Os experimentos impõem diversos vínculos sobre as teorias e o MP até agora está de acordo com todos eles. Um exemplo é o parâmetro $\rho$ relacionado a uma simetria custodial do MP. Pode-se mostrar [16] que a relação (3.63) é válida a nível árvore:

$$
\rho=\frac{m_{W}}{m_{Z} \cos \theta_{W}}
$$

e usando 3.29 temos então: $\rho=1$. Com correções radioativas esse parâmetro é corrigido para aproximadamente, $\rho \approx 1,002$, compatível com a experiência. A figura 3.8 mostra a relação de $\rho$ com a massa do $W$. 


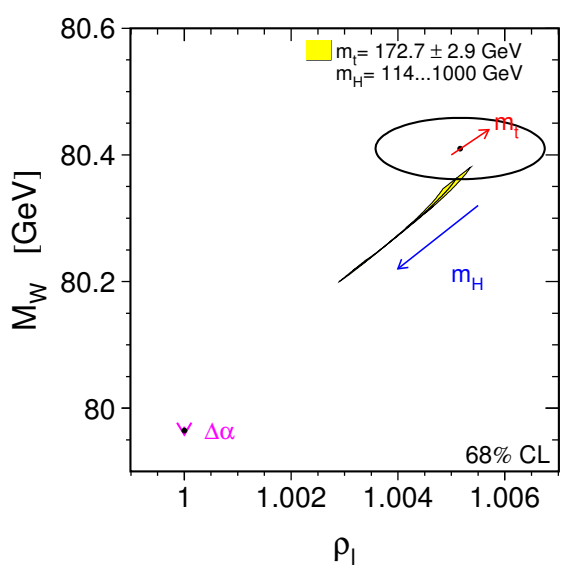

Figura 3.8: Relação entre a massa do $W$ e o parâmetro $\rho$, [23].

Há ainda questões sobre as anomalias do MP, uma delas mostra que o fato do número de famílias de léptons ser igual ao de quarks é essencial para o cancelamento de anomalias. De maneira que se descobrirmos no futuro uma quarta geração de um deles, esperamos encontrar também do outro, formando mais uma geração de férmions. Algumas dessas questões permanecem sem explicação como por exemplo, o motivo por existirem na natureza três cores ou três gerações. Não se entende a origem da geração das misturas de quarks, o padrão das massas, a dinâmica da violação de $\mathrm{CP}$, entre outras.

Então, apesar do enorme sucesso experimental, acredita-se que o Modelo Padrão não é a palavra final na descrição das interações de gauge por motivos que passamos a descrever.

\subsection{Problemas com o MP}

Vimos que alguns setores do MP foram testados severamente e a compatibilidade entre teoria e experimento é surpreendente. Ainda assim, existem questões abertas nesses setores e há ainda outros setores que sabemos pouco a respeito. O chamado setor de quebra espontânea de simetria ainda é obscuro, de maneira que o LHC poderá dizer mais a respeito do mecanismo de quebra para consolidar ou descartar o mecanismo usado no MP. Alguns dos problemas que o MP apresenta são comentados aqui e através deles pode-se obter limites da massa do Higgs como passamos a ver.

\subsubsection{Tivialidade e Estabilidade}

De maneira bem sucinta, a Trivialidade no MP está ligado ao acoplamento $\lambda$ das autointerações do Higgs. O parâmetro $\lambda$ é renormalizável e para que a teoria seja válida em altas energias, é necessário que $\lambda$ seja finito. O crescimento de $\lambda$ com a escala de energia, 
$Q$ é dado ${ }^{6}$ pela função $\beta$

$$
\frac{1}{\lambda(Q)}=\frac{1}{\lambda\left(Q_{0}\right)}-\frac{3}{4 \pi^{2}} \log \left(\frac{Q^{2}}{Q_{0}^{2}}\right)
$$

somando a série geométrica temos

$$
\lambda(Q)=\frac{\lambda\left(Q_{0}\right)}{1-\frac{3}{4 \pi^{2}} \log \left(\frac{Q^{2}}{Q_{0}^{2}}\right)}
$$

então, a medida que a escala de energia cresce, $Q \rightarrow \infty, \lambda$ também cresce e diverge independente do valor na escala de referência, $\lambda\left(Q_{0}\right)$. A saída é ter $\lambda\left(Q_{0}\right)=0$, que não é interessante, pois se trata de uma teoria trivial.

Impondo que o acoplamento quártico, $\lambda$ seja finito a altas energias pode-se calcular um limite para a massa do Higgs. Se tomarmos como referência o VEV, $Q_{0}=v$ e pedindo que o MP seja válido até a escala de Planck, $\Lambda \approx 10^{16} \mathrm{GeV}$, tem-se:

$$
M_{H}^{2}<\frac{8 \pi^{2} v^{2}}{3 \log \left(\frac{\Lambda^{2}}{v^{2}}\right)} \Rightarrow M_{H}<160 \mathrm{GeV}
$$

sendo que este cálculo é ingênuo, pois para escalas de energias tão altas seria necessário levar em conta correções de ordem mais alta e efeitos não-perturbativos. O parâmetro $\Lambda$ é interpretado como a escala até onde o modelo é válido.

Uma outra questão associada ao acoplamento quártico diz respeito a estabilidade da teoria. Como discutido na seç̧ão 2.3.1, é necessário que $\lambda>0$ para que a teoria seja limitada inferiormente e possua ao menos um vácuo. Equivalentemente, pode-se dizer que a simetria deve ser quebrada para energias menores que o VEV,

$$
V(v)<V(0)
$$

Novamente estudando o comportamento de $\lambda$ mas agora para pequenas energias, mostra-se [14] que

$$
\lambda(\Lambda)=\lambda(v)+\frac{1}{16 \pi^{2}}\left[-12 \frac{m_{t}^{2}}{v^{2}}+\frac{3}{16}\left(2 g^{4}+\left(g^{2}+g^{\prime 2}\right)\right)\right] \log \left(\frac{\Lambda^{2}}{v^{2}}\right)
$$

e usando correções de 2 loops chega-se em outro limite para a massa do Higgs ${ }^{7}$,

$$
M_{H}>130+2\left(m_{t}-170\right)
$$

sendo que com os dois limites, (3.66) e (3.69) temos uma região favorecida pelo Modelo Padrão para a massa do Higgs. Curiosamente, esta região não foi descartada pelo LEP

\footnotetext{
${ }^{6}$ Veja [14] por exemplo.

${ }^{7}$ Novamente assumindo que o MP seja válido até $10^{16} \mathrm{GeV}$.
} 
e pelo Tevatron. Os primeiros dados do LHC ainda não foram suficientes para declarar uma descoberta do bóson de Higgs e até a data em que esta dissertação foi escrita (início de 2012) o Higgs não foi encontrado. Caso o canal de decaimento principal do Higgs seja $H \rightarrow \gamma \gamma$, o LHC poderá ver o bóson, mas somente com o acúmulo de mais dados devido ao fundo do MP. Apesar de não ter sido observado um desvio de $5 \sigma$, há um acúmulo de eventos nos dados tomados tanto pelo ATLAS quanto pelo CMS no LHC. Ambos os experimentos mostram um pico na mesma região, que é justamente a favorecida pelo MP. A figura 3.9 mostra as regiões já previamente excluídas pelo LEP e Tevatron e a figura 3.10 mostra as novas buscas feitas pelo LHC.

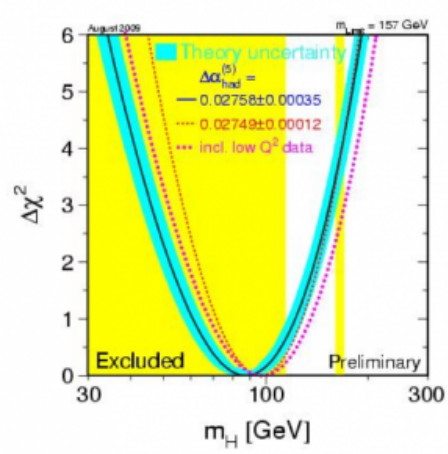

Figura 3.9: Regiões excluídas pelo LEP e Tevatron para a massa do bóson de Higgs.

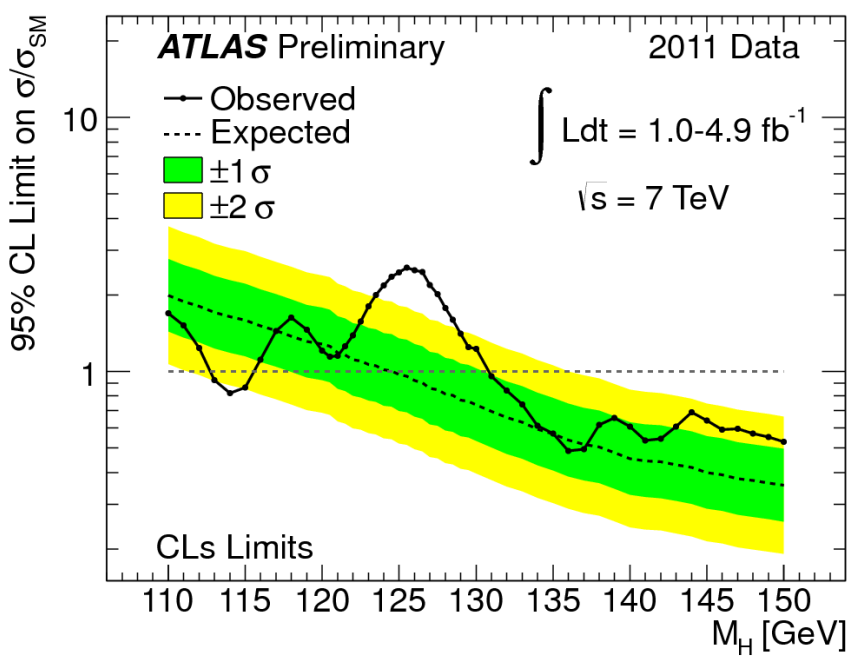

Figura 3.10: Regiões analisadas pelas colaborações ATLAS no LHC para a massa do Higgs [24]. As regiões nas quais a curva cheia está abaixo da curva tracejada são regiões de exclusão com $95 \%$ de nível de confiança.

Na busca pelo Higgs no LHC, novas regiões foram descartadas com nível de exclusão maior que 95\%, estas são as regiões abaixo de onde a linha forte cruza a linha tracejada. A janela onde o Higgs pode estar está ficando menor, o LHC pretende ter luminosidade suficiente para observá-lo ou descartá-lo até o final de 2012 [24]. 


\subsubsection{Naturalidade, o Problema da Hierarquia}

Como vimos na discussão sobre o setor de Higgs, em 3.6, a massa do Higgs é um parâmetro renormalizável da lagrangeana do MP, então as correções radioativas alteram a massa do Higgs. O maior problema relacionado às correções consiste no caráter escalar do bóson de Higgs.

Os férmions de Dirac no MP são férmions quirais (respeitam a simetria quiral [12]), uma vez que na lagrangeana não há termos de massa para eles. Eles adquirem massa através da quebra de simetria no mecanismo de Higgs, mas não há um termo explícito do tipo, $m \bar{\psi} \psi$ na lagrangeana e devido ao caráter espinorial dos campos, as correções às massas dos férmions são multiplicativas, veja por exemplo [2]. Com isso temos

$$
\delta m \propto m_{0} \ln \left(\frac{\Lambda^{2}}{m_{0}^{2}}\right)
$$

onde $\delta m$ é a correção à massa do férmion, $\Lambda$ é o corte da teoria e $m_{0}$ é a massa nua, como apresentada na lagrangeana antes da renormalização. Mas se $m_{0}=0$ como no caso do MP, tem-se que $\delta m=0$ em todas as ordens de perturbação. Diz-se que os férmions são protegidos pela simetria quiral de modo que não há correções radioativas às massas deles. Há uma proteção análoga para o caso dos bósons de gauge, uma vez que eles não possuem massa antes da quebra, deixando invariante a simetria de gauge.

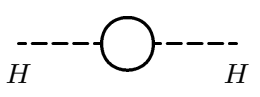

Figura 3.11: Diagrama correspondente às correções à massa do Higgs devido às contribuições fermiônicas no loop.

Porém como todo escalar numa teoria quântica de campos, o Higgs não possui uma simetria desse tipo. As correções a sua massa são aditivas, de maneira que diagramas como o da figura 3.11 dão origem à correções do tipo

$$
\delta M_{H}^{2}=\frac{-N_{C}\left|y_{t}\right|^{2}}{8 \pi^{2}}\left[\Lambda^{2}+m_{t}^{2}-3 m_{t}^{2} \ln \left(\frac{\Lambda^{2}+m_{t}^{2}}{m_{t}^{2}}\right)+\ldots\right]
$$

sendo que aqui consideramos apenas o efeito do quark top, uma vez que por ter maior massa ele se acopla mais fortemente com o Higgs e é quem mais contribui nas correções nos diagramas tipo 3.11. Aqui, $\Lambda$ novamente representa o corte da teoria, $y_{t}$ é o Yukawa do top e $m_{t}$ sua massa. O resto da conta representa termos finitos no limite $\Lambda \rightarrow \infty$. Porém vemos claramente a dependência quadrática com o corte $\Lambda$ e diferentemente dos férmions, tomar $M_{H}=0$ não adianta. Não há nada que proteja a massa do Higgs das correções perturbativas. Somando outras principais contribuições a correção à massa do 
Higgs é dada por (3.72).

$$
\delta M_{H}^{2}=\frac{3}{8 \pi v^{2}} \Lambda^{2}\left(6 m_{W}^{2}+3 m_{Z}^{2}+3 M_{H}^{2}-12 m_{t}^{2}\right) \approx-\left(\frac{\Lambda}{0.7 \mathrm{TeV}} 200 \mathrm{GeV}\right)^{2}
$$

Como foi visto anteriormente, o MP favorece através de limites teóricos, uma massa para o Higgs na escala de poucos centos de $G e V$, em torno de $120-160 \mathrm{GeV}$. De maneira que para que o MP seja natural, ou seja, não haja grandes cancelamentos, $\Lambda$ deve ser da ordem de $1 \mathrm{TeV}$ e não da escala de Planck. Esses cancelamentos são conhecidos como ajuste fino de uma teoria e espera-se que eles não tenham que ocorrer para acerto de parâmetros. Esse problema é conhecido no MP como o problema da hierarquia. Como a mais alta energia que conhecemos está na escala de Planck, por que o MP seria válido somente até a escala $\mathrm{TeV}$ ?

Diversas saídas para essa questão foram apresentadas em teorias além do Modelo Padrão. Uma das mais belas idéias foi a introdução de uma nova simetria, a superssimetria ${ }^{8}$ que introduz novos escalares na escala $\mathrm{TeV}$ com acoplamentos específicos que cancelam às contribuições fermiônicas à massa do Higgs. Modelos como Little Higgs também introduzem novas partículas na escala TeV mas com o mesmo spin das partículas do MP. Há ainda outros modelos que também abordam o problema da hierarquia, mas com outras motivações centrais, mas em todos os casos, partículas com massa de poucos TeV são esperadas e o LHC tem potencial para observá-las. Esta é uma das razões que os físicos esperam nova física na escala $\mathrm{TeV}$.

\subsubsection{Unitariedade}

Uma outra questão de particular interesse no presente trabalho é a unitariedade no espalhamento dos bósons longitudinais eletrofracos, a saber, a unitariedade em altas energias do espalhamento $W_{L}^{+} W_{L}^{-} \rightarrow W_{L}^{+} W_{L}^{-}$. Sendo que de acordo com o Teorema da Equivalência [17], esse espalhamento em altas energias é equivalente ao espalhamento dos bósons de Goldstone. Como vimos no estudo do Mecanismo de Higgs no MP, os graus de liberdade que dariam origem aos bósons de Goldstone foram engolidos pelos bósons de gauge e estes se tornaram massivos ganhando mais um grau de liberdade. Esse grau de liberdade longitudinal adicional é associado ao bóson de Goldstone.

Para um espalhamento desse tipo, $2 \rightarrow 2$, a secção de choque diferencial é dada por

$$
\frac{d \sigma}{d \Omega}=\frac{1}{64 \pi^{2} s}|\mathcal{M}|^{2}
$$

sendo $s$ a energia quadrática do centro de massa da colisão. Decompondo a amplitude $\mathcal{M}$

\footnotetext{
${ }^{8}$ Veja [19] para um bom livro introdutório.
} 
em ondas parciais com os polinômios de Legendre, tem-se

$$
\mathcal{M}=16 \pi \sum_{l=0}^{\infty}(2 l+1) P_{l}(\cos \theta) a_{l}
$$

e integrando então a secção de choque diferencial e usando a ortogonalidade dos Polinômios de Legendre, temos a secção de choque total dada por (3.75).

$$
\begin{aligned}
\sigma= & \frac{8 \pi}{s} \sum_{l=0}^{\infty} \sum_{l^{\prime}=0}^{\infty}(2 l+1)\left(2 l^{\prime}+1\right) a_{l} a_{l}^{*} \int_{-1}^{1} d(\cos \theta) P_{l}(\cos \theta) P_{l^{\prime}}(\cos \theta)= \\
= & \frac{16 \pi}{s} \sum_{l=0}^{\infty}(2 l+1)\left|a_{l}\right|^{2}
\end{aligned}
$$

Fazendo uso do Teorema Óptico, podemos rescrever essa secção de choque como

$$
\sigma=\frac{1}{s} \operatorname{Im}[\mathcal{M}(\theta=0)]=\frac{16 \pi}{s} \sum_{l=0}^{\infty}(2 l+1)\left|a_{l}\right|^{2}
$$

e olhando para a amplitude (3.74) vemos que para $\theta=0, \mathcal{M}=16 \pi \sum_{l=0}^{\infty}(2 l+1) a_{l}$ e então imediatamente temos:

$$
\left|a_{l}\right|^{2}=\operatorname{Im}\left(a_{l}\right)
$$

equivalentemente temos:

$$
\left[\operatorname{Re}\left(a_{l}\right)\right]^{2}+\left[\operatorname{Im}\left(a_{l}\right)\right]^{2}=\operatorname{Im}\left(a_{l}\right) \Rightarrow\left[\operatorname{Re}\left(a_{l}\right)\right]^{2}+\left[\operatorname{Im}\left(a_{l}\right)-\frac{1}{2}\right]^{2}-\frac{1}{4}=0
$$

onde reconhecemos a equação da circunferência de raio, $R=\frac{1}{2}$, centrada no ponto do plano complexo, $\left(0, \frac{1}{2}\right)$. Então a condição (3.77) é equivalente à (3.79).

$$
\left|R e\left(a_{l}\right)\right| \leq \frac{1}{2}
$$

Para que o espalhamento seja unitário em todas as ordens de perturbação, vamos considerar a onda partial $J=0$, dada por $a_{0}$, [16]. No limite $M_{H}^{2} \ll s$ temos:

$$
\begin{aligned}
a_{0}= & \frac{1}{16 \pi s} \int_{-s}^{0} d t|\mathcal{M}|=-\frac{1}{16 \pi s} \int_{-s}^{0} d t \frac{M_{H}^{2}}{v^{2}}\left(2+\frac{M_{H}^{2}}{s-M_{H}^{2}}+\frac{M_{H}^{2}}{t-M_{H}^{2}}\right) \\
& \approx-\frac{M_{H}^{2}}{16 \pi v^{2}}\left(2+\mathcal{O}\left(\frac{M_{H}^{2}}{s}\right)\right)
\end{aligned}
$$

e no limite de altas energias temos $\mathcal{O}\left(\frac{M_{H}^{2}}{s}\right) \approx 0$ e então:

$$
a_{0} \rightarrow_{s \gg M_{H}^{2}}-\frac{M_{H}^{2}}{8 \pi v^{2}}
$$


com a condição (3.79) temos finalmente um limite na massa do Higgs dado por (3.82).

$$
M_{H}^{2} \leq 4 \pi v^{2} \Rightarrow M_{H} \lesssim 870 \mathrm{GeV}
$$

Note que isso não significa que o bóson de Higgs não pode ser mais pesado que esse valor, mas isso implicaria que a teoria de perturbações não seria mais válida. Aplicando o mesmo raciocínio, mas para outros espalhamento é possível obter outros vínculos como $M_{H}^{2}<780(\mathrm{GeV})^{2}$. Ainda estudando este espalhamento, mas analisando o limite inverso, em que a energia é baixa, $s \ll M_{H}^{2}$ temos

$$
a_{0} \rightarrow_{s \ll M_{H}^{2}}-\frac{s}{32 \pi v^{2}}
$$

e com a condição de unitariedade obtemos

$$
\sqrt{s_{C}}<1.7 \mathrm{TeV}
$$

sendo $s_{C}$ a escala onde a perturbatividade é violada. Aqui vemos a justificativa da espera de nova física na escala TeV. Mesmo que não seja o Higgs que restaure a unitariedade nos espalhamentos de bósons vetoriais, alguma partícula com massa nessa escala deve aparecer para fazer o trabalho. Caso o Higgs seja pesado demais ou simplesmente não exista, as novas partículas previstas pelas teorias além do MP serão responsáveis pela restauração e deverão aparecer nos eventos com poucos TeV de energia no centro de massa. O LHC, um colisor com $\sqrt{s}=14 \mathrm{TeV}$ tem enorme potencial para produzir tais partículas, o desafio então será o de encontrá-las, este é o tema pricipal desta dissertação.

\subsubsection{Outras Imperfeições}

Além dos problemas que citamos anteriormente, o MP apresenta outras questões que não são bem resolvidas por ele. Algumas diretamente relacionadas à sua estrutura e outras que ele simplesmente não aborda. Entre elas podemos citar algumas aqui, como:

- Violação de CP forte: experimentalmente os vínculos impostos sobre violação de CP nas interações fortes são muito grandes, de maneira que há nenhuma evidência que as interações fortes violem CP. Todavia, um termo invariante de Lorentz e renormalizável que viola CP pode ser escrito na lagrangeana da QCD. É estranho que tenhamos que desconsiderar esse termo somente por imposição experimental, sem dinâmica. Há propostas, como de Peccei e Quinn [18] para ditar uma dinâmica que inibe a violação de CP introduzindo uma nova partícula, o áxion;

- Oscilações de Neutrinos: dados colhidos de neutrinos atmosféricos, solares e mais recentemente de usinas nucleares sugerem que neutrinos trocam de sabor ao viajarem. 
Nenhum outro processo indica violação de sabor no setor leptônico, mas a oscilação de neutrinos é um sinal bem claro e indica que os neutrinos possuem massa;

- Matéria Escura: observações astronômicas indicam a presença de um tipo de matéria conhecida como matéria escura que não interage com a matéria usual pelas interações do MP. O MP não possui nenhum candidato direto para a matéria escura;

- Bariogênese: cosmologicamente, sabe-se que o Universo apresenta uma assimetria entre matéria e anti-matéria. Todas as estruturas que observamos são formadas pela matéria usual, mas se no Big Bang ambos os tipos de matéria foram criados simetricamente, o que pode ter causado essa assimetria? A violação de CP é uma causa possível, mas somente a fase complexa presente no MP não é capaz de explicar toda essa diferença;

- Há um grande número de parâmetros não conhecidos no MP, que necessita de entradas experimentais para computá-los. O setor das massas das partículas e seus padrões de mistura não tem uma dinâmica que explica sua estrutura;

- O setor de quebra espontânea da simetria eletrofraca é ainda obscuro experimentalmente. No MP é dado por um escalar que adquire um VEV não nulo, porém o potencial é completamente arbitrário e postulado na teoria, sendo que o ingrediente principal, o bóson de Higgs, ainda não foi observado.

Devido a essas questões o Modelo Padrão não é tomado como a teoria final a respeito das interações de gauge entre as partículas elementares. Além disso, conhecemos pelo menos mais uma interação fundamental, a Gravidade. Em todos os processos de interesse do MP, a gravidade se torna desprezível, porém sabemos que ela existe e uma teoria que busca descrever todas as forças conhecidas deverá levá-la em conta. Acredita-se que nova física apareça no LHC, antes da escala de $1 \mathrm{TeV}$, mostrando o caminho para as teorias além do Modelo Padrão.

Muitas teorias nasceram para tentar explicar as questões abertas do MP, como superssimetria [19], technicolor [20], teorias com Dimensões Extras [21], Modelos de Little Higgs [22], etc. É difícil uma teoria que sozinha resolva todas as questões levantadas nessa secção. Normalmente aborda-se a questão da unitariedade e o problema da hierarquia como principais e devido a existência de novas partículas nessas teorias, normalmente apresenta-se candidatos naturais para matéria escura, por exemplo. Mas como vimos os experimentos do MP impuseram inúmeros vínculos nos parâmetros do modelo, de modo que essas novas teorias devem satisfazer esses vínculos também. O espaço de parâmetros ajustáveis fica menor à medida que mais experimentos são realizados no LHC, sendo que no futuro próximo muitos novos modelos serão descartados pela experiência ou fortificados por ela. 


\section{Capítulo 4}

\section{Introdução a Modelos com Dimensões Extras}

Como vimos no capítulo 3 o Modelo Padrão, apesar dos muitos sucessos experimentais, apresenta algumas dificuldades, principalmente relacionadas com o setor de quebra espontânea da simetria eletrofraca. Uma das dificuldades relacionadas a esse setor é a descoberta $^{1}$ do bóson de Higgs ${ }^{2}$, que tem um papel fundamental no processo de quebra da simetria eletrofraca no MP. Há ainda outras dificuldades como por exemplo, o postulado do potencial que introduzimos para o termo de interação do Higgs na equação (2.39), ou seja, não há nenhuma dinâmica que explique o surgimento deste potencial. Mesmo que o Higgs do MP apareça, é de interesse de muitos físicos estender o MP para tentar solucionar algumas de suas questões que não são completamente entendidas.

Uma possível classe de modelos que tentam estender o MP são os chamados Modelos com Dimensões Extras (DE). Nesse tipo de modelo, acredita-se que o espaço-tempo tenha mais do que as $4(3+1)$ dimensões conhecidas, podendo ter uma ou mais dimensões extras espaciais. Existem diferentes tipos de Modelos com DE's, que podem diferir quanto ao número de DE's, na geometria dessas (métrica) e ainda em como a matéria se propaga nas novas dimensões. Aqui apresenta-se uma introdução a esses tipos de modelos e como eles podem resolver alguns problemas do MP, mas o tratamento detalhado de todos eles não é o interesse dessa dissertação. Apresenta-se aqui somente algumas ideias comuns a todos os diferentes modelos e que são importantes para abordar por exemplo o problema da hierarquia no MP. Trata-se com um pouco mais de detalhe o Modelo com Dimensões Extras Curvas - Warped Extra Dimensions [25]. Para referências muito mais completas

\footnotetext{
${ }^{1}$ Até o momento em que essa dissertação foi escrita não houve nenhum anúncio da descoberta do bóson. Mas há um excesso de eventos nas colaborações ATLAS e CMS do LHC que indicam um Higgs com aproximadamente $125 \mathrm{GeV}$, mas não necessariamente o Higgs do MP.

${ }^{2}$ Esta é uma nota adicionada na revisão dessa dissertação. No dia 4 de Julho de 2012 o CERN apresentou uma conferência com seus resultados indicando a presença de uma nova resonância com massa de aproximadamente $125 \mathrm{GeV}$. Nessa conferência ainda não se pode dizer se a ressonância era o Higgs do MP, pois ainda não se havia medido seus acoplamentos entre outras coisas.
} 
sobre esses assuntos veja por exemplo [21], [26] e [27] e suas referências.

\subsection{Motivação}

Assim como muitos outros modelos além do Modelo Padrão, os Modelos com Dimensões Extras tentam resolver o problema da hierarquia. Um modo de abordar essa questão é tentar entender o motivo pelo qual a gravidade é tão mais fraca que as outras forças. Uma das primeiras propostas ${ }^{3}$ para essa explicação propõe o conceito de branas e bulk. As branas são objetos com $p$ dimensões, as chamadas $p$-branas, onde toda a matéria e os campos de gauge podem se propagar. No nosso caso, tem-se uma brana quadridimensional no qual $S U(3)_{C} \times S U(2)_{L} \times U(1)_{Y}+$ matéria podem se propagar. E o bulk é a região entre duas branas conectadas pela dimensão extra (que é portanto compacta, DEC) e somente a gravidade pode se propagar ali $^{4}$ explicando o motivo pela fraqueza da gravidade.

Um jeito de ver isso é observar que a gravitação se dilui na dimensão extra, enquanto os outros campos ficam concentrados nas branas. Seja então uma teoria com $n$ dimensões extras espaciais e compactas e seja $M_{*}$ a escala fundamental nesta teoria (a escala de Planck) e $M_{P} \sim 10^{19} \mathrm{GeV}$ é a escala de Planck como é vista pela teoria 4D. O volume de uma DEC é proporcional ao seu raio, de modo que se há $n$ DEC o volume das dimensões extras é tal que:

$$
V_{n} \propto r^{n}
$$

e da relatividade geral, sabe-se que a ação quadridimensional de Einstein é dada por,

$$
S_{4}=-\frac{M_{P}^{2}}{16 \pi} \int d x^{4} \sqrt{g_{4}} R_{4}
$$

sendo $R$ o escalar de curvatura e $g$ o determinante da métrica, que neste caso tomamos como sendo plana em todas as dimensões, ou seja,

$$
d s^{2}=\eta_{\mu \nu} d x^{\mu} d x^{\nu}-\delta_{a b} d y^{a} d y^{b}=g_{M N} d x^{M} d x^{N}
$$

sendo, $\eta$ a métrica de Minkowski e como sempre os índices gregos se referem às dimensões tradicionais, enquanto que a coordenada y é usada para descrever a posição na dimensão extra (aqui só colocamos uma para ilustrar) e as letras latinas maiúsculas correm de $0,1,2,3,5$ sendo 5 a nova dimensão compacta.

\footnotetext{
${ }^{3}$ Uma outra grande motivação para modelos com DE's vem da teoria das cordas que precisam de mais dimensões espaciais para que a teoria quântica de campos seja matematicamente consistente. Mas aqui não faremos essa abordagem, simplesmente emprestamos algumas ideias das Cordas e aplicamos para Física de Partículas.

${ }^{4}$ Esse é um ponto onde as teorias podem diferir, a determinação de quais campos podem se propagar nas DE's. Para ilustrar como a gravidade pode parecer fraca, admite-se aqui por facilidade que somente ela se propaga no bulk.
} 
Assumindo que a ação em $4+n$ dimensões tem a mesma forma, obtem-se então

$$
S_{4+n}=-\frac{M_{*}^{2+n}}{16 \pi} \int d x^{4+n} \sqrt{g_{4+n}} R_{4+n}
$$

e devido à métrica simplificada (4.3) o termo $\sqrt{g_{4+n}} R_{4+n}$ simplesmente adquire um fator de escala $r^{n}$ que podemos integrar nas DEC's usando $d x^{n}$ e então obter o volume dessas dimensões, proporcional a $r^{n}$. Com isso a ação 4D e a nova ação se relacionam por

$$
M_{*}^{2+n} r^{n}=M_{P}^{2}
$$

e dessa forma, a escala fundamental $M_{*}$ pode ser bem menor que a aparente $M_{P}$. Como a constante de Newton da gravitação se relaciona com a escala fundamental com o inverso do quadrado, ou seja,

$$
G_{N} \sim \frac{1}{M_{P}^{2}}=\frac{1}{M_{*}^{2+n} r^{n}}
$$

vemos que a gravitação é fraca, não porque a escala de Planck é muito alta, mas sim porque a gravidade se propaga em todas as dimensões, diluindo sua intensidade. Usando $M_{P}=1,2 \cdot 10^{19} \mathrm{GeV}$, e pedindo que $M_{*}$ seja da ordem de $1 \mathrm{TeV}$ tem-se que o tamanho das novas DEC's é dado por

$$
r \approx 2 \cdot 10^{-17} \cdot 10^{\frac{32}{n}}
$$

para $n=1$ a dimensão extra teria um tamanho muito grande, $r \sim 10^{8} \mathrm{~km}$ e já teria sido notada e mesmo para $n=2$ experimentos tipo Cavendish de alta precisão conseguiram excluir $r \sim 2 \mathrm{~mm}$. Para $n>2$, os experimentos ainda não podem afirmar nada mas seria necessário pelo menos 3 novas dimensões extras para explicar a fraqueza da gravidade neste cenário. Outra possibilidade é aumentar a escala fundamental, por exemplo, $M_{*} \sim$ $10 \mathrm{TeV}$. Uma outra saída ainda é mudar a métrica que é o que vamos explorar com um pouco mais de detalhe. Mas essa breve discussão já ilustra como a introdução de novas dimensões espaciais pode ajudar a explicar o motivo da gravidade parecer mais fraca em relação às outras forças.

\subsection{Modos de Kaluza-Klein}

Uma característica comum de modelos com dimensões extras compactas diz respeito a torre de novas partículas originadas pela presença das DEC's. Para entender melhor a origem dessa torre, tomemos uma dimensão extra compacta de comprimento $L$ e raio $R=\frac{L}{2 \pi}$. A priori a dimensão extra pode ser infinita como as outras $3+1$ conhecidas, semi-infinita ou compacta. Sendo que neste último caso ela pode ser um círculo ou um intervalo. Porém pode-se relacionar esses dois casos através do $S^{1} / Z_{2}$ orbifold, veja por 
exemplo [26]. Aqui escolhemos uma dimensão extra circular para ilustração. O momento de uma partícula é então dado por $P_{M}=\left(P_{\mu}, P_{5}\right)$. Impondo condições de periodicidade nesta dimensão $y$ temos que

$$
\phi(y)=\phi(y+2 \pi R)
$$

onde $\phi$ é a função de onda da partícula de modo que seja proporcional ao termo de onda plana $e^{i P_{M} x^{M}}=e^{i P_{\mu} x^{\mu}} \cdot e^{i p_{5} y}$ válido para a métrica (4.3). Usando isso tem-se então:

$$
e^{-i P_{5} y}=e^{-i P_{5}(y+2 \pi R)} \Rightarrow e^{-i P_{5} 2 \pi R}=1=e^{ \pm i 2 n \pi}, n \in \mathbb{N}
$$

e assim

$$
P_{5}= \pm \frac{n}{R}
$$

e lembrando a relação de dispersão, tem-se:

$$
P_{M} P^{M}=M^{2}+\left(P_{5}\right)^{2}=M^{2}+\frac{n^{2}}{R^{2}}
$$

de onde pode-se ver a existência de uma torre de partículas, os chamados modos de KaluzaKlein. Tomando o fóton em 5D como exemplo, tem-se $M_{\gamma}=0$ então no $n$-ésimo modo (excitação) sua massa será $\frac{n}{R}$. Neste caso, onde a métrica é plana, o espaçamento entre dois modos quaisquer é fixo, ou seja, a diferença na massa de dois modos consecutivos é sempre $\frac{1}{R}$. Pode-se mudar esse espaçamento alterando a forma da métrica como veremos na secção 4.3 .

\subsection{Dimensões Extras Curvas}

Neste tipo de teoria, a métrica 5-dimensional não é mais plana como em (4.3) e utilizando a curvatura da dimensão extra, ela é capaz de abordar o problema da hierarquia através da supressão da escala de Planck. Nesta secção, que é somente uma introdução ao assunto, iremos estudar o comportamento dos campos nesta nova dimensão para podermos abordar a questão da unitariedade do espalhamento dos bósons vetoriais do MP, para uma revisão mais detalhada do modelo e suas consequência veja por exemplo [28].

Temos então um modelo 5D sendo que a nova dimensão espacial é um intervalo relacionado com o círculo através do orbifold $S^{1} / Z_{2}$, ou seja, associamos os pontos $y$ da DE com $-y$ a fim de no futuro conseguirmos definir uma quiralidade para os férmions. Dessa forma, a coordenada $y$ da DE varia de $y=0$ até $y=\pi R$ e a métrica utilizada é da forma:

$$
d s^{2}=e^{-2 k|y|} \eta_{\mu \nu} d x^{\mu} d x^{\nu}+d y^{2}
$$

onde $\eta$ é a métrica plana de Minkowski, $y$ é a coordenada da nova dimensão e adicionamos um fator conforme dependende da coordenada $y$, de modo que para um valor de $y$ fixo 
a fatia 4D do espaço é plana, sendo $k$ o fator de curvatura da DE. Então fatiando-se a quinta dimensão obtemos branas quadri-dimensionais com a geometria de Minkowski. No ponto $y=0$ localizamos a brana de Planck, uma brana 4D cuja escala de energia é da ordem da escala de Planck e no outro extremo, $y=\pi R$ está localizada a brana $\mathrm{TeV}$, cuja escala é da ordem da escala de energia eletrofraca afim de resolver o problema da hierarquia (usando o decaimento exponencial presente na métrica).

Na proposta original do Modelo, [25] somente a gravidade se propagava na DE para resolver o Problema da Hierarquia, aqui iremos supor que os campos de gauge e os campos fermiônicos do MP também se propagam no bulk, o que abre a possibilidade de abordar questões como o problema das massas dos férmions. Devemos então estudar o comportamento desses campos na dimensão extra e para isso precisamos entender como fazer a redução dimensional da lagrangeana da teoria, ou seja, como integrar a lagrangeana na nova dimensão:

$$
S=\int d^{4} x d y \mathcal{L}_{5 D}=\int d^{4} x \mathcal{L}_{4 D}
$$

e assim obter a ação 4-dimensional que conhecemos para identificar com o MP. Comecemos então pelos bósons de gauge.

\subsubsection{Bósons de Gauge no bulk}

Para os campos de gauge 5D temos:

$$
S_{\text {gauge }}^{5 D}=\int d^{4} x d y \sqrt{g}\left[-\frac{1}{4} g^{M N} g^{R S} F_{M R} F_{N S}\right]
$$

sendo $g$ o determinante da métrica (necessário para que o elemento da integração seja invariante). Podemos escrever a métrica como

$$
g_{M N}=\left(\begin{array}{cc}
e^{-2 k y} & 0 \\
0 & -1
\end{array}\right)
$$

então $\sqrt{g}=e^{-4 k y}$, assim

$$
\begin{gathered}
S_{\text {gauge }}^{5 D}=\int d^{4} x d y\left[-\frac{e^{-4 k y}}{4}\right]\left\{e^{4 k y} F_{\mu \nu} F^{\mu \nu}+2 e^{+2 k y} \eta^{\mu \nu} g_{55} F_{\mu 5} F_{\nu 5}\right\}= \\
=-\frac{1}{4} \int d^{4} x d y\left\{F_{\mu \nu} F^{\mu \nu}-2 e^{-2 k y} F_{\mu 5} F^{\mu 5}\right\}
\end{gathered}
$$

$\operatorname{com} F_{\mu 5} F^{\mu 5}=\left(\partial_{\mu} A_{5}-\partial_{5} A_{\mu}\right)\left(\partial^{\mu} A^{5}-\partial^{5} A^{\mu}\right)=\partial_{\mu} A_{5} \partial^{\mu} A^{5}+\partial_{5} A_{\mu} \partial^{5} A^{\mu}-2 \partial_{5} A_{\mu} \partial^{\mu} A^{5} \Rightarrow$ $F_{\mu 5} F^{\mu 5}=\partial_{\mu}\left(A_{5} \partial^{\mu} A_{5}\right)-A_{5} \partial^{2} A_{5}+\partial_{5} A_{\mu} \partial_{5} A^{\mu}-2 \partial_{5} A_{\mu} \partial^{\mu} A^{5}$. E como uma derivada total 
4-dimensional não afeta a ação, temos ${ }^{5}$

$$
S_{\text {gauge }}^{5 D}=\int d^{4} x d y\left\{-\frac{1}{4} F_{\mu \nu} F^{\mu \nu}+\frac{1}{2} e^{-2 k y}\left[-A_{5} \partial^{2} A_{5}+\partial_{5} A_{\mu} \partial_{5} A^{\mu}-2 \partial_{5} A_{\mu} \partial^{\mu} A^{5}\right]\right\}
$$

e integrando por partes o termo $\partial_{5} A_{\mu} \partial_{5} A^{\mu}$ temos que o termo de superfície não se anula uma vez que a dimensão extra é finita, precisamos então de condições de contorno nas bordas:

$$
S_{\text {gauge }}^{5 D}=\int d^{4} x \int_{0}^{\pi R} d y\left\{-\frac{1}{4} F_{\mu \nu} F^{\mu \nu}+\frac{1}{2} e^{-2 k y}\left[-A_{5} \partial^{2} A_{5}-2 \partial_{5} A_{\mu} \partial^{\mu} A^{5}-A_{\mu} \partial_{5} \partial^{5} A^{\mu}\right]\right\}
$$

com as condições

$$
\begin{cases}\left.A_{5}\left(x^{\mu}, y\right)\right|_{y=0, \pi R} & =0 \\ \left.\partial_{5} A_{\mu}\left(x^{\mu}, y\right)\right|_{y=0, \pi R} & =0\end{cases}
$$

podemos então expandir o campo $A_{\mu}(x, y)$ em modos de Kaluza-Klein (KK), de maneira que:

$$
A_{\mu}(x, y)=\frac{1}{\sqrt{\pi R}} \sum_{n} A_{\mu}^{(n)}(x) \chi^{(n)}(y)
$$

sendo que $\chi$ satisfaz a ortogonalidade

$$
\int_{0}^{\pi R} \frac{d y}{\pi R} \chi^{(n)}(y) \chi^{(m)}(y)=\delta_{n m}
$$

usando a expansão (4.20) e a condição de ortogonalidade (4.21) podemos integrar a ação $5 \mathrm{D}$ em $y$ obtendo

$$
S_{\text {gauge }}=\int d^{4 x}\left[\sum_{n}\left(-\frac{1}{4} F_{\mu \nu}^{(n)}(x) F^{(m) \mu \nu}(x)\right)+\sum_{n} \frac{1}{2} A_{\mu}^{(n)}(x) A^{(m) \mu}(x) \int d y \chi_{n} \partial_{5} e^{-2 k y} \partial_{5} \chi_{n}\right]
$$

e com essa integração queremos obter a ação 4D conhecida

$$
S_{4 D}=\int d^{4 x}\left[\sum_{n}\left(-\frac{1}{4} F_{\mu \nu}^{(n)}(x) F^{(m) \mu \nu}(x)\right)+\sum_{n} \frac{1}{2} m_{n}^{2} A_{\mu}^{(n)}(x) A^{(n) \mu}\right]
$$

então $\chi$ deve satisfazer a equação:

$$
-\partial_{5}\left(e^{-2 k y} \partial_{5} \chi_{n}(y)\right)=m_{n}^{2} \chi_{n}(y)
$$

\footnotetext{
${ }^{5}$ Note que somente a derivada $\partial_{\mu}($ campo $)$ quando integrada nas dimensões espaciais conhecidas que não afeta a ação, pois sempre pedimos que os campos caiam com a distância, o que não é verdade para a DE.
} 
cuja solução mais geral é uma combinação de funções de Bessel:

$$
\chi_{n}(y)=\frac{e^{k y}}{N_{n}}\left[J_{1}\left(\frac{m_{n}}{k} e^{k y}\right)+\beta Y_{1}\left(\frac{m_{n}}{k} e^{k y}\right)\right]
$$

com $N_{n}, \beta$ e $m_{n}$ constantes que podem ser determinadas a partir das C.C. (4.19) e a normalização (4.21). Para que o campo $A_{\mu}$ possua um modo zero $(n=0)$ é necessário que $\chi$ seja par sob $\mathbb{Z}_{2}$ e também também satisfaça a C.C.

$$
\begin{cases}\chi_{n}(y) & =\chi_{n}(-y) \\ \left(\frac{d}{d y} \chi_{n}(y)\right)_{0, \pi R} & =0\end{cases}
$$

pois queremos identificar o modo zero com o campo de gauge do MP. No limite $m_{n} \ll k$ temos

$$
m_{n} \approx\left(n-\frac{1}{4}\right) \pi k e^{-k \pi R}
$$

de onde vemos a torre de modos KK, com massas dadas por $m_{n}$.

\subsubsection{Férmions no bulk}

Para estudar o comportamento dos férmions na nova dimensão é necessário modificar a lagrangeana 4D que conhecemos. A relação de anticomutação das matrizes $\gamma$ está relacionada com a métrica do espaço-tempo de maneira que as matrizes de Dirac 5dimensionais devem ser bem definidas. Sejam então as matrizes $\Gamma^{A}$ definidas por:

$$
\Gamma^{A}=E_{B}^{A} \gamma^{B}
$$

com $\gamma^{A}=\left(\gamma^{\mu}, \gamma^{5}\right)$ sendo $\gamma^{\mu}$ as matrizes de Dirac tradicionais. $E_{B}^{A}$ é o vierbein inverso, ou seja, o inverso do tensor $e_{B}^{A}$ que nos ajuda a escrever a métrica $g_{M N}$ em função da métrica plana, $\eta_{M N}$ através de

$$
g_{M N}=e_{M}^{A} \eta_{A B} e_{N}^{B}
$$

e a equação para $E_{B}^{A}$ é dada por

$$
E_{B}^{A} e_{C}^{B}=\delta_{C}^{A}
$$

usando a métrica de RS (4.15) temos que

$$
\begin{aligned}
e_{B}^{A} & =\operatorname{diag}\left(e^{-k y}, e^{-k y}, e^{-k y}, e^{-k y}, 1\right) \\
E_{B}^{A} & =\operatorname{diag}\left(e^{k y}, e^{k y}, e^{k y}, e^{k y}, 1\right)
\end{aligned}
$$


com isso temos a ação de um férmion em 5D dada por

$$
S_{\text {férmion }}^{5 D}=\int d^{4} x d y \sqrt{g}\left\{i \bar{\psi} \Gamma^{A} \frac{\partial_{A}-\overleftarrow{\partial}_{A}}{2} \psi+M_{\psi} \bar{\psi} \psi\right\}
$$

com $\psi=\psi(x, y)$ e usando (4.28) temos

$$
\begin{aligned}
S_{f}^{5 D}= & \int d^{4} x d y \sqrt{g}\left\{\frac{i}{2} E_{B}^{A} \bar{\psi} \gamma^{B}\left(\partial_{B}-\overleftarrow{\partial}_{B}\right) \psi+M_{\psi}\right\}= \\
= & \int d^{4} x d y \sqrt{g}\left\{e^{-3 k y}\left(\bar{\psi}_{R} \not \partial \psi_{R}+\bar{\psi}_{L} \not \partial \psi_{L}\right)+e^{-4 k y} M_{\psi}\left(\bar{\psi}_{L} \psi_{R}+\bar{\psi}_{R} \psi_{L}\right)+\right. \\
& \left.-\frac{1}{2}\left[\bar{\psi}_{L}\left(e^{-4 k y} \partial_{5}+\partial_{5} e^{-4 k y}\right) \psi_{R}-\bar{\psi}_{R}\left(e^{-4 k y} \partial_{5}+\partial_{5} e^{-4 k y}\right) \psi_{L}\right]\right\}
\end{aligned}
$$

com $\psi_{L, R}=\frac{1}{2}\left(1 \mp \gamma_{5}\right) \psi$. Assim como no caso dos bósons de gauge vamos decompor os campos fermiônicos em modos de KK:

$$
\psi_{L, R}=\frac{1}{\sqrt{\pi R}} \sum_{n} \psi_{L, R}^{(n)}(x) e^{2 k y} f_{L, R}^{(n)}(y)
$$

com as funções $f_{L, R}$ satisfazendo a normalização (4.35).

$$
\frac{1}{\pi R} \int_{0}^{\pi R} d y e^{k y} f_{L, R}^{(n)} f_{L, R}^{(m)}=\delta_{n m}
$$

Usando a decomposição (4.34) na ação (4.33) podemos integrar na dimensão extra para obter

$$
\begin{aligned}
S_{f}= & \sum_{n} \int d^{4} x\left\{\bar{\psi}_{L}^{(n)} i \not \partial \psi_{L}^{(n)}+\bar{\psi}_{R}^{(n)} i \not \partial \psi_{R}^{(n)}+\right. \\
& +\int d y M_{\psi}\left[\left(f_{L}^{*(n)} \bar{\psi}_{L}^{(n)} \psi_{R}^{(m)} f_{R}^{(m)}+f_{R}^{*(n)} \bar{\psi}_{R}^{(n)} \psi_{L}^{(m)} f_{L}^{(m)}\right)+\right. \\
& \left.\left.-\left(f_{L}^{*(n)} \bar{\psi}_{L}^{(n)} \partial_{5} \psi_{R}^{(m)} f_{R}^{(m)}-f_{R}^{*(n)} \bar{\psi}_{R}^{(n)} \partial_{5} \psi_{L}^{(m)} f_{L}^{(m)}\right)\right]\right\}
\end{aligned}
$$

e juntando novamente as componentes do campo $\psi=\psi_{L}+\psi_{R}$ reescrevemos a ação como em (4.37).

$$
\begin{aligned}
S_{f}= & \int d^{4} x\left\{\bar{\psi}^{(n)} i \not \partial \psi^{(n)}+\int d y\left[\bar{\psi}_{L}^{(n)} \psi_{R}^{(m)}\left(M_{\psi} f_{L}^{*(n)} f_{R}^{(m)}-f_{L}^{*(n)} \partial_{5} f_{R}^{(m)}\right)+\right.\right. \\
& \left.\left.+\bar{\psi}_{R}^{(n)} \psi_{L}^{(m)}\left(M_{\psi} f_{R}^{*(n)} f_{L}^{(m)}+f_{R}^{*(n)} \partial_{5} f_{L}^{(m)}\right)\right]\right\}
\end{aligned}
$$

Também como no caso bosônico queremos que essa ação reduzida seja igual à ação 
fermiônica 4D conhecida:

$$
S_{f}^{4 D}=\sum_{n} \int d^{4} x\left(\bar{\psi}_{n} i \not \partial+m_{n} \bar{\psi}_{n} \psi_{n}\right)
$$

para que isso seja satisfeito devemos impor que

$$
\begin{array}{r}
\int d y\left[\bar{\psi}_{L}^{(n)} \psi_{R}^{(m)}\left(M_{\psi} f_{L}^{*(n)} f_{R}^{(m)}-f_{L}^{*(n)} \partial_{5} f_{R}^{(m)}\right)+\bar{\psi}_{R}^{(n)} \psi_{L}^{(m)}(\right. \\
\left.\left(M_{\psi} f_{R}^{*(n)} f_{L}^{(m)}+f_{R}^{*(n)} \partial_{5} f_{L}^{(m)}\right)\right]= \\
=m_{n}\left(\bar{\psi}_{n}^{L}+\bar{\psi}_{n}^{R}\right)\left(\psi_{n}^{L}+\psi_{n}^{R}\right)
\end{array}
$$

de onde tiramos as relações que as funções $f_{L, R}$ devem satisfazer para que a ação obtida pela redução dimensional seja idêntica a ação conhecida 4D:

$$
\begin{cases}\left(M_{\psi}-\partial_{5}\right) f_{R}^{(n)}= & m_{n} e^{k y} f_{L}^{(m)} \\ \left(M_{\psi}+\partial_{5}\right) f_{L}^{(n)}= & m_{n} e^{k y} f_{R}^{(m)}\end{cases}
$$

para resolver esse sistema acoplado costuma-se utilizar a parametrização $M_{\psi}=c k$, ou seja, a massa do férmion é proporcional à curvatura $k$ com $c$ uma constante de proporcionalidade de ordem 1, pois não queremos introduzir um novo problema de hierarquia. Usando essa parametrização e desacoplando ${ }^{6}$ as equações obtemos como solução geral:

$$
\left\{\begin{array}{l}
f_{L}^{(n)}(y)=\sqrt{\frac{m_{n}}{k}} e^{k y}\left[a_{L}^{(n)} J_{c+\frac{1}{2}}\left(\frac{m_{n}}{k} e^{k y}\right)+b_{L}^{(n)} Y_{c+\frac{1}{2}}\left(\frac{m_{n}}{k} e^{k y}\right)\right] \\
f_{R}^{(n)}(y)=\sqrt{\frac{m_{n}}{k}} e^{k y}\left[a_{R}^{(n)} J_{c-\frac{1}{2}}\left(\frac{m_{n}}{k} e^{k y}\right)+b_{R}^{(n)} Y_{c-\frac{1}{2}}\left(\frac{m_{n}}{k} e^{k y}\right)\right]
\end{array}\right.
$$

de onde podemos observar novamente a aparição de uma torre de partículas, para cada excitação $n$.

Agora que vimos uma brevíssima ${ }^{7}$ introdução da Teoria Quântica dos Campos para bósons de gauge e férmions numa teoria 5 dimensional com dimensão extra curva podemos ver como essa teoria é capaz de abordar a questão da unitarização de espalhamentos de bósons de gauge do MP.

\subsubsection{Espalhamento de Bósons de Gauge em Teorias com Dimen- sões Extras}

Como foi discutido anteriormente, o bóson de Higgs tem um papel fundamental no MP. Além da participação na quebra espontânea da simetria eletrofraca, ele também é res-

\footnotetext{
${ }^{6}$ Para mais detalhes da conta veja por exemplo [28].

${ }^{7}$ Muitos aspectos da teoria com dimensões extras curvas não foram abordados aqui, como por exemplo a relação entre geração de massa para os férmions e sua localização no bulk e o mesmo para os bósons. Há ainda muitas teorias com dimensões extras muito interessantes como Dimensões Extras Universais que possuem um candidato direto para a matéria escura que não foram tratados nesta dissertação, para mais vide as referências citadas no início do capítulo.
} 
ponsável por garantir a unitariedade do espalhamento de bósons longitudinais como por exemplo, $W_{L} W_{L} \rightarrow W_{L} W_{L}$. Sem o Higgs, a amplitude deste espalhamento cresceria com $E^{2}$ violando a unitariedade em $1.8 \mathrm{TeV}$, pois os diagramas envolvendo somente os bósons de $S U(2) \times U(1)$ cancelam somente a dependência com $E^{4}$. Nos modelos com dimensões extras sem Higgs (ou com Higgs muito pesado) os bósons da torre de KK são os responsáveis por garantir a unitariedade desse espalhamento através de um processo recursivo. Cada excitação anterior tem a unitariedade garantida pela excitação seguinte.

Para conseguir cancelar a dependência quadrática com a energia é necessário que as constantes de acoplamento da teoria 5-dimensional e as massas dos bósons vetoriais satisfaçam algumas relações que iremos obter. Por simplicidade usaremos aqui a métrica plana e não a de RS que mostramos anteriormente e trataremos o espalhamento elástico dos $n$-ésimos modos de KK dos bósons de gauge longitudinais, $n_{a}+n_{b} \rightarrow n_{c}+n_{d}$ com $a, b, c$ e $d$ os índices de gauge como em [16]. Definimos então as grandezas cinemáticas:

$$
\begin{aligned}
\epsilon_{\mu} & =\left(\frac{p_{\mu}}{M_{n}}, \frac{E}{M_{n}} \frac{\vec{p}}{|\vec{p}|}\right) \\
p_{\mu}^{\text {in }} & =\left(E, 0,0, \pm \sqrt{E^{2}-M_{n}^{2}}\right) \\
q_{\mu}^{\text {out }} & =\left(E, \pm \sqrt{E^{2}-M_{n}^{2}} \sin \theta, 0, \pm \sqrt{E^{2}-M_{n}^{2}} \cos \theta\right)
\end{aligned}
$$

essa definição é tal que a altas energias temos $\epsilon_{\mu} \approx E, p_{\mu} \approx E$ e o propagador é da ordem de $E^{-2}$. Com isso para energias bem maiores que a massa do $W\left(E \gg M_{W}\right)$, que é o regime do LHC que iremos tratar na análise no capítulo 6, a amplitude do espalhamento $W_{L} W_{L} \rightarrow W_{L} W_{L}$ em função da energia fica:

$$
\mathcal{A}=\mathcal{A}^{(4)} \frac{E^{4}}{M_{n}^{4}}+\mathcal{A}^{(2)} \frac{E^{2}}{M_{n}^{2}}+\mathcal{A}^{(0)}+\mathcal{O}\left(\frac{M_{n}^{2}}{E^{2}}\right)
$$

sendo $\mathcal{A}^{(n)}$ coeficientes dessa expansão. Argumentamos que no MP o termo $\mathcal{A}^{(4)}$ é nulo somente levando em conta os bósons de gauge eletrofracos, mas o termo $\mathcal{A}^{(2)}$ só se anula caso a contribuição do Higgs seja considerada. É possível mostrar, como em [29] que o coeficiente $\mathcal{A}^{(4)}$ no espalhamento dos bósons $n$ é dado por:

$$
\mathcal{A}^{(4)}=i\left(g_{n n n n}^{2}-\sum_{k} g_{n n k}^{2}\right)\left[\epsilon^{a b e} \epsilon^{c d e}\left(3+6 \cos \theta-\cos ^{2} \theta\right)+2\left(3-\cos ^{2} \theta\right) \epsilon^{a c e} \epsilon^{b d e}\right]
$$

onde $g_{n n n n}$ é a constante de acoplamento do vértice quártico dos bósons $n$ (no diagrama quártico devido ao caráter não-abeliano do grupo de gauge) e $g_{n n k}$ é a constante do vértice cúbico entre os bósons $n$ e o bóson $k$ (nos diagramas tipo $s, t$ e $u$ ), por isso a soma sob $k$.

Para que a amplitude (4.43) seja nula é necessário então que (4.44) seja nulo e para 
isso as constantes de acoplamento dos vértices cúbico e quático devem satisfazer:

$$
g_{n n n n}^{2}=\sum_{k} g_{n n k}^{2}
$$

esse resultado é válido para a métrica plana e para a métrica curva. Porém, agora queremos escrever as constantes de acoplamento usando suas relações com as funções de onda relacionadas com as expansões dos campos como foi feito na secção 4.3.1. Sendo assim as expressões seguintes são válidas somente para dimensões extras planas, mas a generalização é imediata. Seja então as funções de onda $f_{n}(y)$, as relações entre $f_{n}$ e as constantes dos vértices cúbico e quártico são dadas por:

$$
\begin{aligned}
g_{m n k} & =g_{5} \int d y f_{m}(y) f_{n}(y) f_{k}(y) \\
g_{m n k l}^{2} & =g_{5}^{2} \int d y f_{m}(y) f_{n}(y) f_{k}(y) f_{l}(y)
\end{aligned}
$$

podemos então usar a relação de completeza para as funções $f_{n}$ :

$$
\sum_{k} f_{k}(y) f_{k}(z)=\delta(y-z)
$$

assegurando que a igualdade entre as constantes (4.45) seja satisfeita, uma vez que

$$
\begin{aligned}
\sum_{k} g_{n n k}^{2}= & g_{5}^{2} \sum_{k}\left(\int_{0}^{\pi R} d y f_{n}^{2}(y) f_{k}(y)\right)\left(\int_{0}^{\pi R} d z f_{n}^{2}(z) f_{k}(z)\right)= \\
& =g_{5}^{2} \int_{0}^{\pi R} d y \int_{0}^{\pi R} d z f_{n}^{2}(y) f_{n}^{2}(z) \delta(y-z)= \\
& =g_{5}^{2} \int_{0}^{\pi R} d y f_{n}^{4}(y)=g_{n n n n}^{2}
\end{aligned}
$$

onde usamos a relação (4.47) na primeira passagem, integramos usando $\delta(y-z)$ e finalmente reconhecemos a constante $g_{n n n n}$ dada por (4.46). Sendo assim, se as constantes do modelo são tais que (4.46) seja satisfeita o coeficiente $\mathcal{A}^{(4)}$ é nulo e o quadrático é dado por:

$$
\mathcal{A}^{(2)}=\frac{i}{M_{n}^{2}}\left(4 g_{n n n n} M_{n}^{2}-3 \sum_{k} g_{n n k}^{2} M_{k}^{2}\right)\left(\epsilon^{a c e} \epsilon^{b d e}-\sin ^{2}\left(\frac{\theta}{2}\right) \epsilon^{a b e} \epsilon^{c d e}\right)
$$

e aqui aparece a recursividade necessária para o cancelamento, pois para que $\mathcal{A}^{(2)}=0$ é necessário que haja uma relação entre as constantes cúbicas e quárticas e as massas dos 
modos excitados para que $4 g_{n n n n} M_{n}^{2}-3 \sum_{k} g_{n n k}^{2} M_{k}^{2}=0$, ou seja,

$$
g_{n n n n} M_{n}^{2}=\frac{3}{4} \sum_{k} g_{n n k}^{2} M_{k}^{2}
$$

para que essa relação seja satisfeita é necessário que as condições de contorno para as funções $f_{n}$ sejam do tipo Neumann ou Dirichilet. Se aplicarmos (4.46) e (4.50) para o caso de nosso interesse, $W_{L} W_{L} \rightarrow W_{L} W_{L}$, obtemos:

$$
\begin{aligned}
g_{W W W W} & =g_{W W Z}^{2}+g_{W W \gamma}^{2}+\sum_{k} g_{W W k}^{2} \\
4 g_{W W W W} M_{W}^{2} & =3\left[g_{W W Z}^{2} M_{Z}^{2}+\sum_{k} g_{W Z K}^{2} M_{k}^{2}\right]
\end{aligned}
$$

sendo $k$ um bóson de KK neutro (por conservação de carga) com massa $M_{k}$.

Garantimos então com essas relações que $\mathcal{A}^{(2)}$ e $\mathcal{A}^{(4)}$ são nulos, mas para garantir que toda a amplitude seja nula é necessário ainda que $\mathcal{A}^{0}=0$. Isso ilustra o caráter não renormalizável da teoria 5-dimensional implicando um crescimento linear com a energia. Sendo assim, o modelo com dimensões extras é uma teoria efetiva que possui validade até uma certa escala $\Lambda$ que é o corte da teoria. A unitariedade dos espalhamentos dos bósons longitudinais é violada em uma escala que pode ser obtida através de estimativas de $\Lambda$ e a partir dessa escala uma nova teoria deve entrar em cena. As estimativas dependem do modelo usado (da métrica e do número de DE's) então não faremos nenhuma aqui.

Temos como objetivo agora estudar a fenomenologia de um modelo com uma excitação pesada do bóson $Z$, o chamado bóson $Z^{\prime}$ com massa da ordem de $1 \mathrm{TeV}$. Esse bóson é um dos responsáveis pela unitarização do espalhamento dos $W^{\prime} s$ longitudinais no MP como acabamos de ver, pois há um acoplamento cúbico entre eles com constante $g_{W W Z^{\prime}}$. Mas antes de analisarmos esse decaimento é importante discutirmos um assunto essencial para a análise, os jatos. Como $M_{Z^{\prime}} \gg M_{W}$ os $W^{\prime} s$ produzidos no LHC estarão muito energéticos de maneira que num decaimento do tipo $W \rightarrow j j$ os jatos estarão extremamente próximos dificultando muito a análise. Passamos então a estudar aspectos gerais dos jatos para usar em nossa análise. 


\section{Capítulo 5}

\section{QCD e Jatos}

A QCD como vimos é uma teoria de calibre não-abeliana que descreve as interações fortes entre quarks e glúons. Devido a liberdade assintótica da QCD, podemos tratar quarks e glúons como partículas livres no regime de altas energias (distâncias da ordem de $1 \mathrm{fm}$ ). Porém acredita-se que a QCD seja uma teoria com confinamento ${ }^{1}$ de cor, ou seja, quarks e glúons não podem ser encontrados livres na natureza. Quanto maior a distância entre dois quarks, maior a interação entre eles de maneira que é impossível separá-los. Caso a energia aplicada seja tamanha, um novo par surgirá do vácuo dando origem a novos estados ligados, os hádrons. Os quarks vêm em três cores, normalmente ${ }^{2}$ as usadas são: verde, vermelho e azul.

Os hádrons são as únicas partículas que interagem fortemente. Eles são singletos de cor e são formados por pártons (quarks e glúons), além disso são divididos em dois grupos, os bárions e os mésons. Os bárions são formados por 3 quarks de valência com cores distintas $(q q q)$. Enquanto os mésons são formados por um par quark - anti-quark, de valência $(q \bar{q})$. Além dos quarks de valência, os hádrons também são formados por quarks e glúons do mar, devido a criação e aniquilação de pares de partículas provenientes do vácuo quântico.

Devido ao confinamento, quarks e glúons não são encontrados livres na natureza, então quando eles são criados nos aceleradores acontece o que se chama de parton showering e hadronização. Novas partículas são criadas através de decaimentos e radiação dando origem a um spray colimado de hádrons energéticos que chamamos de jato (singleto de cor). A produção de jatos ocorre de duas maneiras. Quarks podem irradiar glúons, que normalmente estão próximos ao quark inicial (colineares) e são pouco energéticos (moles). Os glúons por sua vez dão origem a um par $q \bar{q}$ ou a um novo par de glúons (devido ao caráter não abeliano da QCD). Isso acontece repeditamente de modo que o párton inicial dá origem a uma cascata de hádrons. Uma outra possibilidade ocorre quando os

\footnotetext{
${ }^{1}$ Confinamento é uma das características da QCD, assim como a liberdade assintótica. A baixas energias, $\lambda_{Q C D} \approx 200 \mathrm{MeV}$, quarks e glúons são confinados em hádrons que passam a ser os graus de liberdade de uma teoria fortemente acoplada.

${ }^{2}$ Alguns norte-americanos mais patriotas podem usar vermelho, branco e azul. Mas normalmente a cor branca remete ao singleto de cor, um bárion por exemplo.
} 
quarks produzidos na colisão encontram-se muito próximos e a medida em que eles vão se afastando a interação entre eles fica cada vez mais forte perturbando o vácuo na região. A densidade de energia na região entre as partículas aumenta de maneira que a criação de um novo par de partículas é energeticamente mais vantajosa do que a separação dos pártons iniciais. Assim novos hádrons são criados e também dão origem a uma cascata de novas partículas originando jatos. É bom salientar que todas as etapas desses fenômenos ocorrem a baixas energias, ou seja, a descrição deles está no regime não-perturbativo da QCD. De modo que para simular tais efeitos usando Métodos de Monte Carlo necessita-se de modelos. O pacote PYTHIA ([30]), por exemplo usa o modelo de cordas Lund (que não está relacionado de nenhuma forma com a teoria das Cordas), o HERWIG ([31] e [32]) usa um Monte Carlo diferente, de maneira que é usual fazer simulações em diferentes programas a fim de se comparar os resultados. Existem ainda outros programas de geração de eventos que fazem a fragmentação dos pártons, o que é bom para a confiabilidade de um resultado, pois espera-se que uma simulação não dependa muito do modelo utilizado no Monte Carlo.

Além da hadronização e da radiação dos pártons ${ }^{3}$, outras partículas provenientes de outras fontes também podem estar presente na região de um jato. Normalmente esses efeitos são chamados na literatura ${ }^{4}$ de Splash-In, Splash-Out, Pile-Up (PU), Multiple Interactions e Underlying Event (UE).

No efeito Splash-In partículas irradiadas pelos prótons no feixe colisor são consideradas como parte de um jato da colisão dura. Enquanto que Splash-Out, é a radiação do evento de interesse que foi deixada de fora pelo algoritmo de busca de jatos. Pile-Up é o efeito de várias colisões ocorrendo simultaneamente no detector. Normalmente um evento interessante é selecionado pelo alto momento transverso da colisão, mas a probabilidade de um choque gerar tal evento cai com a energia (as pdf's caem ${ }^{5}$ rapidamente com o aumento da energia da colisão). Assim a maioria das colisões simultâneas ocorrem a uma energia muito mais baixa que a do evento de interesse, mas alguma partícula vinda dessas colisões pode ser confundida com partículas do evento duro. Deve-se salientar que tais colisões são completamente independentes da colisão principal.

Underlying Event é um efeito devido aos pártons que não se chocaram na colisão dura. Devido a liberdade assintótica, a colisão ocorre entre dois pártons presentes nos prótons e devido ao confinamento de cor, os pártons remanescentes interagem (Multiple Interactions) para formarem singletos de cor. Essas interações normalmente produzem jatos de baixo $p_{t}$ no evento e radiações moles e são correlacionadas pela cor com o evento

\footnotetext{
${ }^{3}$ A radiação pode vir dos estados inicial ou final da colisão dura (Initial State Radiation e Final State Radiation).

${ }^{4}$ Veja por exemplo o glossário de [33] para uma referência rápida.

${ }^{5}$ PDF: Parton Distribution Function, são as funções de distribuição de pártons. Elas descrevem empiricamente o conteúdo de pártons em um hádron, sendo que as contribuições de um quark de valência ou de um párton do mar na seç̧ão de choque de um processo dependem da energia. No LHC, por exemplo, as pdf's são dominadas pelos glúons.
} 
principal.

Todos esses efeitos alteram o funcionamento dos algoritmos de jatos, surgiram então técnicas para minimizar esses efeitos como Pruning ([34] e [35]), Filtering ([36]) e Trimming ([37]). Tais técnicas são descritas na secção 5.1.4, mas antes é necessário estabelecer como determinar um jato.

\subsection{Algoritmos de Reconstrução de Jatos}

Devido ao excesso de partículas num evento hadrônico é necessário que se estabeleça regras para saber se uma partícula pertence a um certo jato do evento ou a um outro que eventualmete pode estar próximo. Assim, é necessário que haja um algoritmo que agrupe as partículas detectadas determinando assim quantos e quais são os jatos no evento. Juntamente com o algoritmo, há ainda um esquema de recombinação que determina como duas partículas são combinadas (o mais simples é a soma dos 4-momentos). Juntos, o algoritmo e o esquema de recombinação determinam uma definição de jato.

Há dois tipos básicos de algoritmos para reconstrução de jatos ${ }^{6}$ : Algoritmos tipo Cone e Algoritmos de Recombinação.

\subsubsection{Algoritmos de Cone}

Este tipo de algoritmo é muito usado nos aceleradores hadrônicos, como o Tevatron e o LHC. Um conjunto de partículas é chamado de jato se satisfazem os requerimentos do algoritmo que passamos a definir. Nos algoritmos tipo Cone define-se uma região formada por um "cone" de raio $R_{\text {cone }}$ e centrado no ponto $\left(y_{C}, \phi_{C}\right)$, sendo $y_{C}$ o valor da rapidez do centro do cone e $\phi_{C}$ seu ângulo azimutal (em torno do feixe). Assim, a $k$-ésima partícula com rapidez e ângulo azimutal $\left(y_{k}, \phi_{k}\right)$ faz parte do jato $C$ se:

$$
k \subset C \text { se } \sqrt{\left(y_{k}-y_{C}\right)^{2}+\left(\phi_{k}-\phi_{C}\right)^{2}} \leq R_{\text {cone }}
$$

e então podemos definir um novo cone centrado em:

$$
\bar{y}_{C} \equiv \frac{\sum_{k \subset C} y_{k} \cdot p_{T, k}}{\sum_{l \subset C} p_{T, l}}, \quad \bar{\phi}_{C} \equiv \frac{\sum_{k \subset C} \phi_{k} \cdot p_{T, k}}{\sum_{l \subset C} p_{T, l}}
$$

com isso o novo centro é dependente dos momentos (tranversos) das partículas associadas ao cone inicial. Se o novo centro (ponderado por $p_{T}$ ) for diferente do centro geométrico, $\left(\bar{y}_{C}, \bar{\phi}_{C}\right) \neq\left(y_{C}, \phi_{C}\right)$, então o cone é centrado em $\left(\bar{y}_{C}, \bar{\phi}_{C}\right)$ e repete-se a iteração até que $\left(\bar{y}_{C}, \bar{\phi}_{C}\right)=\left(y_{C}, \phi_{C}\right)$. Então, em princípio, é possível colocar cones teste em todos espaço

\footnotetext{
${ }^{6}$ Para a história de alguns algoritmos e uma explicação detalhada de inúmeras técnicas que serão somente citadas nesta revisão veja: [38], [39] e [40].
} 
de parâmetros $\left(y_{C}, \phi_{C}\right)$ permitindo que os cones "andem" até que um cone estável seja encontrado.

Introduziou-se então a idéia de sementes (do inglês seeds) que o algoritmo planta nas regiões do detector onde se encontram os maiores depósitos de energia ${ }^{7}$. Porém logo se observou que essa idéia apresentava problemas associados a radiação Infra-Vermelha (emissões moles/colineares). De modo que o algoritmo fica muito susceptível a essas emissões. Há algoritmos (seedless) que contornam o problema e são muito utilizados hoje em dia pelos experimentais devido ao caráter geométrico presente no algoritmo, porém não iremos detalhar esse assunto aqui, veja por exemplo [38], [39] e [43].

\subsubsection{Algoritmos de Recombinação}

Este tipo de algoritmo foi muito usado em aceleradores tipo $e^{+} e^{-}$, como o LEP e seu conceito foi então ampliado para uso nos aceleradores hadrônicos. Seu uso também é muito popular na análise de subestruturas de jatos, que é discutida adiante. A ideia aqui é que duas partículas são agrupadas sempre que a distância entre elas for menor do que um valor de corte (parâmetro do algoritmo). Na sua versão original essa distância dependia da energia das partículas colisoras, mas em aceleradores hadrônicos este conceito é mal definido, uma vez que os pártons interagentes carregam apenas uma fração da energia do hádron no feixe. Neste texto aborda-se somente o uso nos colisores hadrônicos, para referências dos aceleradores leptônicos, como o algoritmo JADE ([44] e [45]) vide [38], [39] e [46].

Em colisões tipo $p p$ normalmente se utiliza quantidades invariantes sob um boost de Lorentz e rotações em torno do eixo do feixe colisor, então introduziu-se as seguintes "distâncias" ([47] e [48]):

$$
d_{i j}=\min \left(p_{T, i}^{2 l}, p_{T, j}^{2 l}\right) \frac{\Delta R_{i j}^{2}}{R^{2}}, \quad \Delta R_{i j}^{2}=\left(y_{i}-y_{j}\right)^{2}+\left(\phi_{i}-\phi_{j}\right)^{2}, \quad d_{i}=p_{T, i}^{2 l}
$$

como antes, $p_{T, i}$ é o momento transverso da $i$-ésima partícula, $\Delta R_{i j}^{2}$ é a distância entre as partículas $i$ e $j$ no espaço de $(y, \phi)$ e $R$ é o parâmetro do algoritmo ${ }^{8}$ ( $l$ será explicado adiante). Aqui cada partícula é considerada como um proto-jato, cria-se então uma lista com todos os proto-jatos e estes são agrupados de acordo com a seguinte iteração:

1. Determine as distâncias $d_{i j}$ e $d_{i}$ para todos pares $i$ e $j$;

2. Ache o mínimo de $d_{i j}$ e $d_{i}$;

3. Se o mínimo for $d_{i j}$, junte (usando um esquema de recombinação) $i$ e $j$ em uma só partícula e volte ao passo 1 ;

\footnotetext{
${ }^{7}$ Para revisões sobre o funcionamento de detectores veja [41] e [42].

${ }^{8}$ Alguns algoritmos usam um $R$ variável, veja [50].
} 
4. Se o mínimo for $d_{i}$, então este proto-jato é promovido a um jato e é removido da lista de proto-jatos a serem recombinados;

5. Repita até que todos os proto-jatos forem recombinados.

Diferentemente dos algoritmos de Cone que trazem uma visão mais geométrica, os algoritmos de recombinação agrupam os pares de partículas mais próximas (de acordo com uma definição de proximidade, dada por (5.3)). De modo que caso se queira analisar a subestrutura dos jatos basta voltar os passos do algoritmo, facilitando a análise e além disso, esses algoritmos são muito populares entre os teóricos e fenomenologistas uma vez que são livres de problemas relacionados à divergências infra-vermelha e colinear.

A recombinação depende não somente da distâncias entre os proto-jatos, mas também de seus momentos transversos. A ordem em que o agrupamento ocorre é determinada pelo valor de $l$ :

- Para $l=1$ o algoritmo recebe o nome de $k_{t}^{9}$ ([47], [48] e [49]). A maior crítica que o algoritmo recebeu na sua forma original (principalmente pelos experimentais) é devido ao longo tempo computacional exigido. Como a iteração deve ser repetida toda vez e somente um par de proto-jatos da lista é recombinado por vez, o tempo de máquina do algoritmo cresce com $N^{3}$, sendo $N$ o número de partículas iniciais na lista. Esse problema foi amenizado recentemente ([51] e [52]) passando para $N \ln N$, usando diagramas de Voronoi para achar o vizinho mais próximo de um ponto $^{10}$. O algoritmo encontra-se no pacote "Fastjet ${ }^{11 "}$ e também contém versões alternativas para o algoritmo $k_{t}$ e outros algoritmos de recombinação adiante. Aqui a recombinação ocorre a partir das partículas com menor momento transverso (moles), primeiro se agrupa as mais moles e por último ficam as mais duras, essa qualidade é muito útil na análise de subestrutura de jatos de partículas pesadas como explicado na secção 5.1.3.

- Para $l=0$ o algoritmo se chama Cambridge/Aschen ([53] e [54]). No C/A, como é chamado, o momento das partículas não é relevante. Somente a distância no plano $(y, \phi)$ determina a ordem da recombinação, nesse aspecto este é o algoritmo de recombinação que mais se assemelha aos tipo Cone devido ao caráter puramente geométrico. Ele é normalmente utilizado na procura de jatos provenientes de partículas pesadas como o quark top, uma vez que os algoritmos de busca dessas partículas usam um $R$ grande ${ }^{12}$. Algoritmos tipo $k_{t}$ possuem uma forma bastante irregular e

\footnotetext{
${ }^{9} \mathrm{Na}$ verdade o nome é $k_{t}$ inclusivo - inclusive $k_{t}$ algorithm, porque há também o exclusive, mas a sequência de recombinação é a mesma, diferindo somente na interpretação dessa sequência. No contexto de colisores, quase sempre que se utiliza o termo $k_{t}$, faz-se referência ao $k_{t}$ inclusivo.

${ }^{10}$ Novamente, os detalhes do algoritmo fogem do objetivo desta revisão, para mais veja [38].

${ }^{11}$ Disponível para download em www.lpthe.jussieu.fr/ $\sim$ salam/fastjet.

${ }^{12}$ Em geral $R=1.5$, enquanto que o valor mais adotado é $R=0.7$. Esse valor padrão foi discutido na
} 
com o uso de um $R$ grande estão sujeitos a contaminação de diferentes fontes como pile-up, multiple events e radiação.

- Para $l=-1$ tem-se o algoritmo anti- $k_{t}$ [56]. Aqui ao contrário do $k_{t}$ as partículas duras são agrupadas primeiro e as mais moles, normalmente provindas de radiação, se juntam depois. Isso significa que o jato cresce de dentro para fora, em torno de "sementes" duras, como os algoritmos de Cone. Mas o $p_{t}$ e a distância angular dos jatos também são levados em conta no algoritmo, isso faz com que ele seja livre de divergências Infra-Vermelhas. Produzindo jatos duros circulares, a topologia dos jatos recombinados por esse algoritmo se assemelha muito a topologia dos algoritmos de Cone. Fazendo do anti- $k_{t}$ um substituto natural para algoritmos de Cone que apresentam divergências IV.

\subsubsection{Subestrutura de Jatos}

Com o funcionamento do LHC será possível testar o setor de quebra espontânea da Simetria EletroFraca e possivelmente teorias além do Modelo Padrão (MP). Em ambos os casos, partículas pesadas como os bósons EletroFracos $(\mathrm{EF})\left(W^{ \pm}, Z^{0}\right)$, o bóson de Higgs e o quark top serão importantes na análise dos eventos. Isso porque em muitas teorias além do MP os acoplamentos das partículas novas com essas partículas pesadas são maiores do que com o resto do espectro do MP.

Caso sinais de nova física sejam detectados no LHC, sua assinatura em geral será a presença das partículas mais pesadas do MP com um alto momento transverso e nas regiões centrais do detector ${ }^{13}$. Tais partículas podem decair em canais leptônicos ou hadrônicos. Os sinais leptônicos são mais limpos que os hadrônicos devido ao ambiente do colisor $p p$ e o enorme background de QCD. Porém o sinal hadrônico normalmente possui uma razão de ramificação maior fazendo com que este canal seja muito importante na busca por nova física. Como veremos no capítulo 6 , quando o $p_{t}$ da partícula primária é muito alto ( $\gtrsim 100 \mathrm{GeV}$ ) os produtos do decaimento saem muito próximos (colimados). De modo que os cortes tradicionais para detecção dessas partículas não são mais aplicáveis.

A baixas energias pede-se que se tenha no evento 2 ou 3 jatos duros com uma distância mínima entre si. Mas para jatos colimados essa distinção fica difícil de ser feita, mesmo a nível numérico aplicando somente os algoritmos de recombinação descritos. O que ocorre é que os algoritmos passam a agrupar todas as partículas em um único jato "gordo".

O que se faz então é procurar por subestruturas no jato principal. Nas análises de subestrutura dos bósons $W$ e $Z$ procura-se por dois subjatos (provenientes dos dois quarks

conferência de Snowmass, na qual se decidiram várias regras que um algoritmo deve satisfazer, quando ainda os algoritmos de jatos estavam nascendo. Para discussões mais detalhadas sobre a conferência e o acordo sobre os algoritmos veja [55].

${ }^{13}$ Longe da direção dos feixes colisores, normalmente pede-se um corte na pseudorapidez, que é invariante por Boosts de Lorentz, $y<2.5$. 
do decaimento) e na identificação do top procura-se três subjatos (um quark bottom, e dois quarks provenientes do decaimento do $W, t \rightarrow W b \rightarrow q q b)$. Nesses casos a massa do jato reconstruído é um bom indicador de sua origem, pede-se que a massa do jato esteja num intervalo centrado na massa conhecida da partícula e a largura do intervalo pode variar com o $p_{t}$ do jato, diz-se que a massa do jato deve estar na janela de massa da partícula conhecida, que é dada por:

$$
\left|M_{\text {jato }}-M_{\text {partícula }}\right| \leq \Delta M_{\text {partícula }}
$$

sendo $2 \cdot \Delta M_{\text {partícula }}$ a largura da janela. O problema com essa análise ingênua vem do enorme background de QCD proveniente das colisões hadrônicas. Mesmo para pártons iniciais sem massa é possível que após a fragmentação um jato de $\mathrm{QCD}^{14}$ possua uma massa grande, igual a massa de uma das partículas pesadas do MP ou ainda maior. Alguns detectores conseguem distinguir jatos provenientes de um quark $b$ devido a sua vida média elevada. O vértice da colisão e o vértice onde o jato foi formado ${ }^{15}$ se encontram separados (da ordem de $1 \mathrm{~mm}$ ), facilitando a identificação. Porém outros jatos de QCD podem ser facilmente confundidos com um jato pesado.

A análise de subestrutura de um jato pode amenizar esse problema. Normalmente, um jato de QCD adquire uma massa grande devido à enorme quantidade de radiação presente nele, essa radiação por sua vez é na maioria dos casos colinear e mole. Num jato pesado por outro lado, estão presentes acúmulos de energia em diferentes células do calorímetro, mas muito próximas. No caso do decaimento $H \rightarrow b \bar{b}$, por exemplo, apesar dos dois quarks serem agrupados em um único jato, ao voltar o processo de recombinação do algoritmo pode-se encontrar dois subjatos duros (por isso o uso de algoritmos de recombinação favorece a análise de subestruturas). No caso da QCD a probabilidade de que existam dois subjatos duros dentro de um único jato é pequena, fazendo com que a chance de se confundir um jato de QCD com um jato pesado diminua.

Existem muitas idéias sobre a análise de subestruturas de jatos, algoritmos como HEPTopTagger ${ }^{16}$ [57] e o Johns Hopkins [58] são especializados na identificação de tops. Uma das primeiras idéias procurava uma queda abrupta de massa na volta de um passo da recombinação [59] no contexto de um Higgs pesado decaindo em $W W$. Posteriormente, no contexto do decaimento hadrônico do Higgs em $b \bar{b}$ e no espalhamento de bósons $W W$, implementou-se uma nova técnica: "Y-splitter" ([36] e [60]). No presente trabalho utilizase uma técnica baseada no trabalho [36] com queda de massa como será detalhado adiante na secção 6 .

Existem ainda outros algoritmos de busca de partículas pesadas, mas todos se resumem

\footnotetext{
${ }^{14}$ Aqui um jato de QCD é todo jato que teve sua origem a partir de um párton, exceto o quark top.

${ }^{15}$ Tipicamente, o jato começa a ser formado onde começa a ocorrer a fragmentação.

${ }^{16}$ Disponível para download em:

http://www.thphys.uni-heidelberg.de/ plehn/index.php?show=heptoptagger\&visible $=$ tools.
} 
a procura de subjatos duros com propriedades que os distinguam dos jatos de QCD. Tais propriedades variam, desde variáveis cinemáticas criadas pelos autores das técnicas ou novos aspectos de jatos como área de jatos, formas e etc. Junto com a análise de subestrutura apareceram na literatura idéias para diminuir efeitos como Pile-Up descritos anteriormente, que foram denomidas genericamente de Grooming, por [61].

\subsubsection{Grooming}

Existem três técnicas mais difundidas com o propósito de refinar a busca por um jato: Filtering, Trimming e Pruning, apelidadas de Grooming. Todas elas começam formando um jato usando algum algoritmo de recombinação: $k_{t}, \mathrm{C} / \mathrm{A}$ ou anti- $k_{t}$. Então, usando algum critério específico recombina-se novamente o jato mas com novos parâmetros ditados pela técnica usada. Isso é feito com o intuito de jogar fora a maior parte de radiação e partículas provenientes de efeitos como Pile-Up e Splash-In, restando somente as partículas duras. Essas técnicas são descritas abaixo de modo muito sucinto, o leitor interessado deve buscar mais detalhes nas referências ([39] e [61]) e nos artigos originais que estão referenciados nas respectivas descrições:

- Filtering com queda de massa [36]:

1. Volte para o último passo do agrupamento do jato $j$. Os dois subjatos são identificados por $j_{1}$ e $j_{2} \operatorname{com} m_{j_{1}}>m_{j_{2}}$;

2. Pare o algoritmo se houver uma queda significante de massa e a separação não for muito assimétrica. Para quantificar essa queda de massa e a simetria entre os jatos introduz-se os parâmetros $\mu$ para queda de massa e $y_{\text {corte }}$ para a simetrização que devem satisfazer (5.5);

$$
m_{j_{1}}<\mu m_{j} \quad e \quad y \equiv \frac{\min \left(p_{T, j_{1}}^{2}, p_{T, j_{2}}^{2}\right) \Delta R_{j_{1}, j_{2}}^{2}}{m_{j}^{2}}>y_{\text {corte }}
$$

3. Se o jato $j$ não passar pelos cortes, redefina o subjato $j_{1}$ como $j$ e repita a interação.

Até agora o processo descreve a técnica de análise de queda de massa somente. A inovação do filtering está no reagrupamento do jato $j$. Quando há uma queda de massa usa-se novamente o algoritmo de recombinação original, mas agora com um novo parâmetro $R$ dado por: $R_{f i l t}=\min \left(0.3, \frac{\Delta R_{j_{1}, j_{2}}}{2}\right)$. Guarda-se então os $N$ subjatos $^{17}$ mais duros e estes formam o novo jato, jogando fora as patículas moles. É possível fazer o processo de reagrupamento sem o critério de queda de massa. Mas muitos autores já se referem à técnica filtering com a técnica de queda de massa implícita.

\footnotetext{
${ }^{17} \mathrm{O}$ parâmetro $N$ é escolhido de maneira a otimizar a análise.
} 
- Prunning ([34] e [35]): Normalmente, mas não obrigatoriamente, é usado com o algoritmo C/A. Dado um jato $j$ já determinado por alguma definição ${ }^{18}$ de jato, repita o procedimento, mas usando o seguinte critério: dois subjatos $i$ e $j$ não serão reagrupados num único jato $p$ (soma de $i$ e $j$ ) se satisfizerem:

$$
z \equiv \frac{\min \left(p_{T, i}, p_{T, j}\right)}{p_{T, p}}<z_{\text {corte }} \text { e } \Delta R_{i j}>D_{\text {corte }}
$$

onde $z_{\text {corte }}$ e $D_{\text {corte }}$ são parâmetros livres. Se tal condição é satisfeita, então descarta-se o jato com menor $p_{t}$ ficando apenas com o mais duro. Continue até que todos os protojatos estejam agrupados ou tenham sido descartados.

- Trimming [37]: Para um dado jato $j$ agrupado com um dos algoritmos de recombinação, reagrupe-o com um parâmetro $R_{\text {sub }}<R$ para identificar subjatos. Descarte todos os subjatos $i$ que satisfazem:

$$
p_{T, i}<f_{\text {corte }} p_{T, j}
$$

Sendo $f_{\text {corte }}$ o parâmetro livre do algoritmo, desse modo os proto-jatos que possuem baixo $p_{t}$ (tipicamente radiação mole) são descartados do jato principal. Neste caso o número de subjatos pode variar ${ }^{19}$ conforme o $p_{t}$ dos subjatos e o corte adotado, para um valor de $f_{\text {corte }}$ alto, somente as partículas mais duras serão guardadas. No caso do decaimento $W \rightarrow j j$, idealmente o algoritmo será capaz de guardar somente os 2 jatos leves que deram origem ao jato gordo (que está na janela de massa do $W$ ) fazendo com que jatos provenientes de radiação sejam desconsiderados e não interfiram na análise. Porém para que isso ocorra, um valor de $f_{\text {corte }}$ otimizado deve ser encontrado comparando as características do sinal desejado e do fundo.

Ainda não há um consenso sobre o uso de grooming nas análises de jatos. Dependendo do propósito, diferentes algoritmos combinados com diferentes técnicas de grooming podem resultar em melhores ou piores análises. A questão sobre qual é o melhor algoritmo e qual é o melhor parâmetro a ser utilizado ainda não foi respondida. Com o LHC muito poderá ser dito sobre o estudo de jatos, uma vez que estes estarão presentes em todos os eventos por serem uma característica intrínseca do colisor hadrônico. Os jatos terão um papel importantíssimo, principalmente nos eventos de interesse que podem conter física além do Modelo Padrão, uma vez que a maior parte dos sinais dessas teorias possuem uma assinatura hadrônica. Estudando somente os casos com assinatura leptônica e energia faltante limita muito o poder de descoberta de novas partículas, porque se exclui a maior parte dos canais de decaimento dessas.

\footnotetext{
${ }^{18}$ Lembre-se, definição de jato não é somente o algoritmo usado, mas também o esquema de recombinação adotado para juntar dois proto-jatos.

${ }^{19}$ No caso de fltering o número de subjatos está fixo.
} 
Apresentou-se aqui um panorâma superficial de como está a área de jatos atualmente, mas além dos assuntos tratados aqui, existem muitos outros aspectos relativos ao estudo de jatos que não foram abordados. Para mais sobre esse assunto, o autor recomenda fortemente a leitura das principais referências aqui citadas para importantes questões não abordadas no presente texto, principalmente os artigos de revisão citados no início do capítulo. 


\section{Capítulo 6}

\section{Simulações e Análises}

Como vimos no capíulo 3, o Modelo Padrão apresenta dificuldades que ainda não foram solucionadas. Uma das propostas atuais para resolver algumas dessas dificuldades é a introdução de novas dimensões espaciais na teoria como vimos no capítulo 4. Sinais da existência de novas dimensões poderão ser inferidos no LHC através da observação de novos bósons vetoriais provenientes da torre de KK. Um desses novos bósons que pode aparecer nos eventos do LHC é o primeiro modo excitado do $Z$, o bóson $Z^{\prime}$.

O sinal característico que analisamos nesta dissertação foi o decaimento do $Z^{\prime}$ em bósons carregados do MP, $Z^{\prime} \rightarrow W^{+} W^{-}$e esses bósons decaindo hadronicamente, $W \rightarrow$ $j j$, com $j$ sendo um dos quarks leves $j=u, d, c, s$. Durante o trabalho nos referimos a este sinal como puramente hadrônico ou simplesmente hadrônico. O sinal semi-leptônico ocorre quando um dos $W^{\prime}$ s decai leptonicamente e o puramente leptônico ou leptônico, quando ambos os bósons decaem em léptons. Esses últimos decaimentos tem como característica a presença de um ou mais léptons duros e energia faltante $\left(\right.$ missing energy, $\mathbb{E}_{t}$ ) proveniente principalmente dos neutrinos que não são detectados pelo LHC.

Os decaimento leptônico e semi-leptônico foram estudados em análises prévias, como em [16] e [62]. Um dos principais motivos do uso desses sinais são suas características, sempre exigindo léptons e $\mathbb{E}_{t}$, o que facilita a detecção devido ao ambiente mais limpo (com menos jatos). Porém o sinal hadrônico apresenta somente jatos no estado final, o que complica muito a busca pela nova ressonância devido ao enorme fundo (background) de QCD. Devido ao ambiente hadrônico do acelerador, os eventos no LHC estão sempre sujeitos à contaminação por radiação como vimos no capítulo 5 .

Ainda assim, o canal hadrônico é muito importante nas buscas por nova física no LHC por sua alta razão de ramificação. Para conseguir usar o sinal hadrônico como um canal viável de busca pelo $Z^{\prime}$ no LHC, fizémos uso de algumas técnicas de subestrutura de jatos descritas na secção 5.1.3 e analisamos como alguns cortes poderiam separar o sinal do fundo. Na presente secção apresenta-se os problemas relacionados ao sinal hadrônico, motiva-se e descreve-se o método utilizado e os resultados são apresentados ao final da análise. 


\subsection{Motivação}

O sinal estudado ${ }^{1}$ neste trabalho ${ }^{2}$ consiste na produção de uma nova ressonância pesada $Z^{\prime}$, com massa da ordem de $1 \mathrm{TeV}$ decaindo em dois bósons $W^{ \pm}$. Na maioria dos eventos de interesse o momento transverso do $Z^{\prime}$ é pequeno, devido à sua grande massa. A figura 6.1 mostra a distribuição aproximada ${ }^{3}$ do momento transverso do $Z^{\prime}$ para massas de 1,2 , 3 e $4 \mathrm{TeV}$.

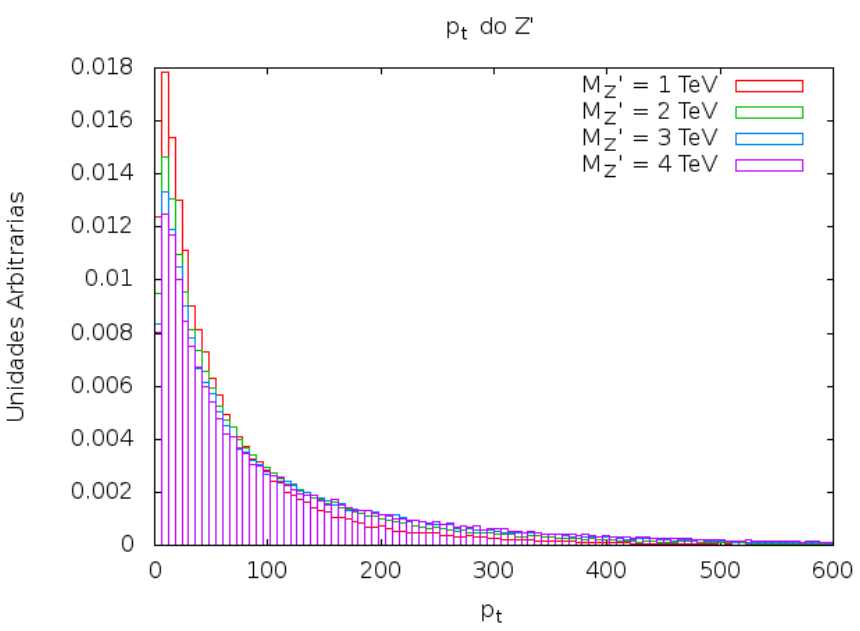

Figura 6.1: Distribuição do momento transverso do $Z^{\prime}$ para $M_{Z^{\prime}}=1,2,3,4 \mathrm{TeV}$. Nota-se que para massas maiores, a distribuição se aproxima mais do zero, pois a maior parte da energia da colisão é usada para dar massa a nova ressonância sobrando pouca energia para se transformar em momento.

Podemos ver que o novo bóson é criado aproximadamente em repouso, como espera-se, pois praticamente toda a energia proveniente da colisão é usada para dar massa ao $Z^{\prime}$ sobrando pouca energia para ele adquirir momento. Quanto maior a massa do novo bóson, mais próxima de zero fica a distribuição do momento transverso na figura 6.1. A fim de conservar momento, o decaimento de uma partícula em duas se dá de maneira que as duas partículas produtos saem em direções (aproximadamente) contrárias (back-to-back) caso a partícula inicial esteja em (aproximadamente) repouso, como podemos ver na figura 6.2.

\footnotetext{
${ }^{1}$ Nesta secção estudamos o comportamento do sinal sem nenhum tipo de corte, ou seja, ainda não fazemos uso do nosso algoritmo que será descrito posteriormente. Inicialmente, estamos interessados em observar algumas características do sinal que permitem distinguí-lo do background.

${ }^{2}$ Veja a secção 6.2 para os programas e pacotes utilizados nas simulações.

${ }^{3} \mathrm{~A}$ distribuição foi gerada pelo parton shower e por isso é uma aproximação da distribuição real.
} 


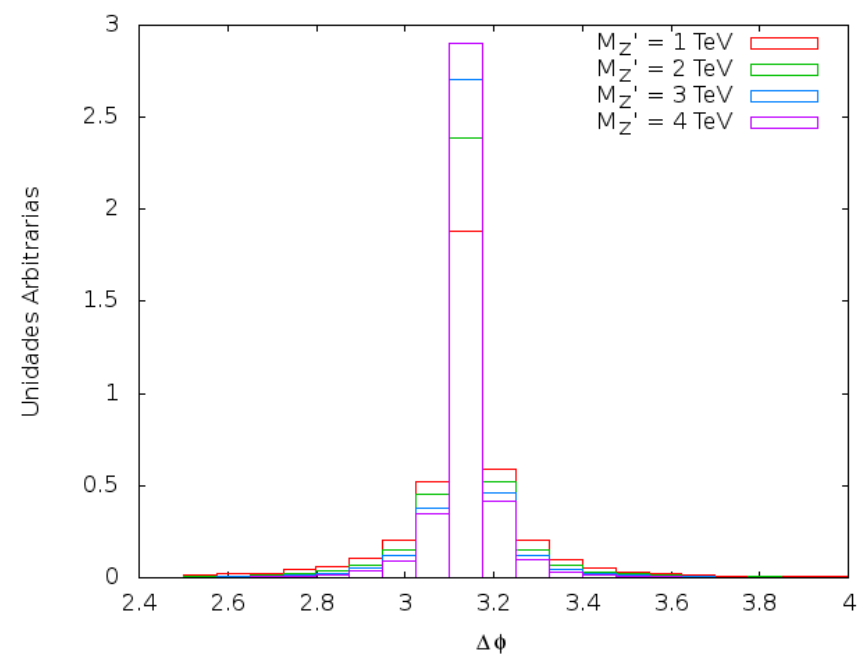

Figura 6.2: Ângulo $\Delta \phi$ entre os dois jatos mais duros dos eventos de sinal $\left(p p \rightarrow Z^{\prime} \rightarrow\right.$ $W W$ ) para $M_{Z^{\prime}}=1,2,3,4 \mathrm{TeV}$. Como esperávamos, quanto maior a massa do $Z^{\prime}$, seu momento é mais próximo de zero e a distribuição de $\Delta \phi$ entre os dois jatos (um de cada $W$ ) é mais estreita em torno de $\Delta \phi=\pi$.

Vemos que o pico da produção dos $W^{\prime} s$ ocorre para $\Delta \phi \approx \pi$. Para massas maiores de $Z^{\prime}$ sua distribuição de $p_{t}$ é mais próxima de zero (perto do repouso) e os dois $W^{\prime} s$ saem em direções contrárias por conservação de momento. Como a massa dos bósons eletrofracos é muito menor que a massa do $Z^{\prime}(80 \mathrm{GeV} \ll 1 \mathrm{TeV})$ os bósons do MP são criados com momento muito grande. As figuras 6.3 e 6.4 mostram respectivamente as distribuições do momento transverso e da massa invariante dos dois jatos gordos mais duros (que identificamos como os jatos originados pelos $W^{\prime} s$ ). A figura 6.5 mostra a reconstrução da massa invariante da soma ${ }^{4}$ dos jatos gordos para diferentes massas do $Z$ '.

${ }^{4} \mathrm{O}$ esquema de recombinação utilizado na identificação dos jatos foi a soma de seus 4-momentos. 


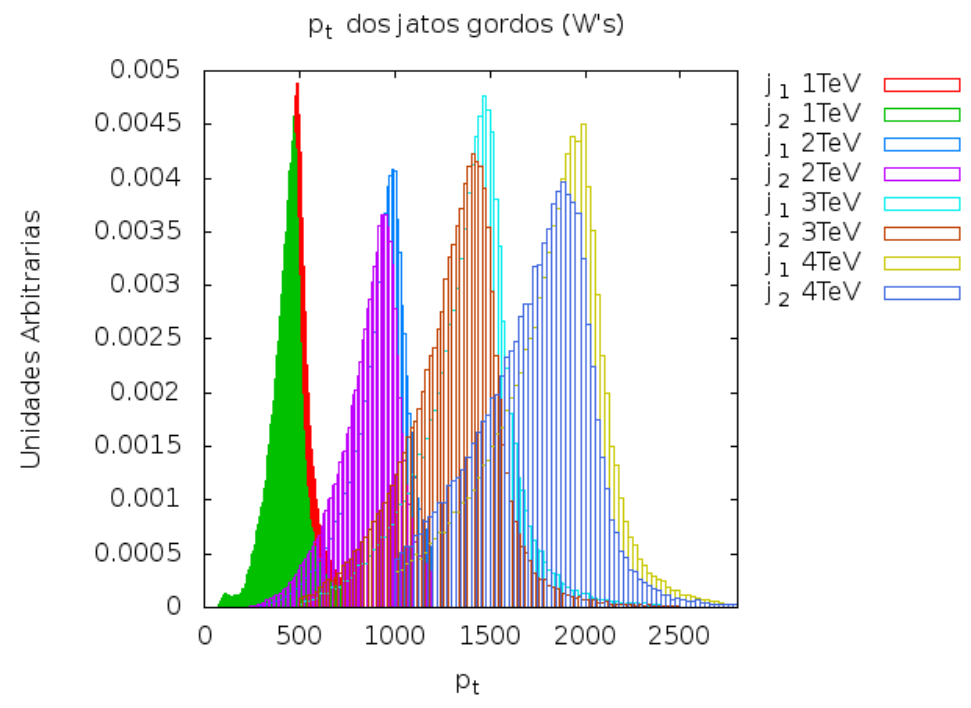

Figura 6.3: Distribuição do momento transverso dos dois jatos gordos encontrados nos eventos $p p \rightarrow Z^{\prime} \rightarrow W W$, para diferentes massas do $Z^{\prime}(1,2,3 \mathrm{e} 4 \mathrm{TeV})$, sendo $j_{i}$ o $i$-ésimo jato mais duro. Pode-se observar que o primeiro jato mais duro (para cada valor de $M_{Z^{\prime}}$ ) está centrado em $\frac{M_{Z^{\prime}}}{2}$ e o segundo (menos massivo) é um pouco menos duro. Para discussão vide texto.
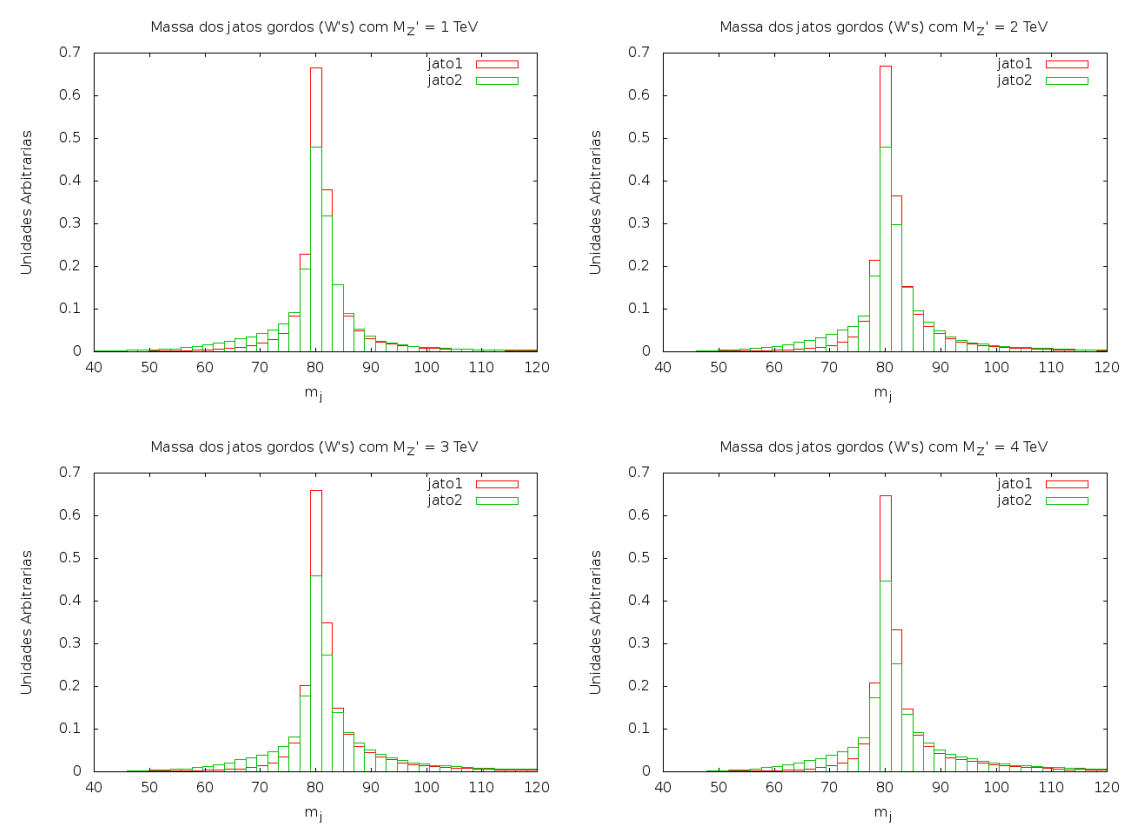

Figura 6.4: Massa Invariante dos dois jatos gordos mais duros em cada evento para $M_{Z^{\prime}}=1,2,3,4 \mathrm{TeV}$. As distribuições reconstroem muito bem a massa do $W$ sendo que ainda não foi imposto nenhum corte, nem mesmo que a massa de cada jato esteja na janela de massa do $W$ (por isso as distribuições se estendem por uma faixa grande de massa). 


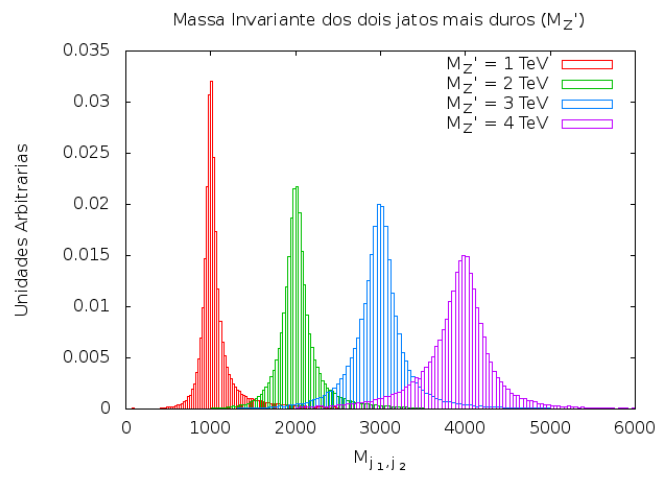

Figura 6.5: Massa invariante da combinação dos dois jatos mais duros dos eventos para $M_{Z^{\prime}}=1,2,3,4 \mathrm{TeV}$. As distribuições estão centradas em $M_{Z^{\prime}}$ sem que houvesse imposição de cortes, como na figura 6.4. Esse aspecto é de fundamental importância para redução de fundos como discutido na secção 6.2 .

Na figura 6.3 podemos observar que os dois jatos mais duros dos eventos estão centrados aproximadamente em $\frac{M_{Z^{\prime}}}{2}$, pois a energia do $Z^{\prime}$ é grande (devido a grande massa de repouso) e portanto as energias de cada $W$ também será grande, da ordem de $\frac{M_{Z^{\prime}}}{2}$ para cada um. Como a massa dos $W^{\prime} s$ é pequena perto de $\frac{M_{Z^{\prime}}}{2}$, praticamente toda a energia é cinética e por isso as distribuições de $p_{t}$ estão centradas nos valores de metade da massa do novo bóson. Os desvios vem do fato de que os gráficos não mostram o $p_{t} \operatorname{dos} W^{\prime} s$ diretamente, mas sim dos dois jatos mais duros em cada evento, fazendo com que efeitos de radiação mudem a forma da distribuição ${ }^{5}$.

As figuras 6.4 e 6.5 reconstroem muito bem ${ }^{6}$ as massas dos $W^{\prime} s$ e do $Z^{\prime}$ mesmo sem imposição de cortes, o que ajuda muito na redução dos fundos como veremos na secção 6.2. Por enquanto ainda não foi imposto que a massa de cada jato gordo esteja perto da massa do $W$ e nem que a massa invariante da soma dos dois jatos reconstrua a massa do $Z^{\prime}$. Faremos isso somente com a imposição de cortes na secção 6.2 .

Como a figura 6.3 mostra e já podíamos esperar, a energia cinética de cada $W$ é bem grande comparada à sua massa. Isso faz com que os produtos dos decaimentos dos $W^{\prime} s$ estejam também com momento transverso alto, diz-se que estão com um alto fator de Lorentz, ou boosted. Se o momento dos $W^{\prime} s$ fosse pequeno os jatos do decaimento $W \rightarrow j j$ estariam suficientemente espaçados para aplicação dos algoritmos de reconstrução de jatos, vistos no capítulo 5. O algoritmo seria capaz de identificar os dois ou mais jatos e assim poderia reconstruir a massa do $W$ para total reconstrução do evento. Porém devido ao alto momento transverso dos $W^{\prime} s$ nos eventos de interesse, os jatos saem extremamente colimados tornando impossível diferenciar os jatos de cada quark. A figura 6.6 mostra a distância no plano $(\phi, y)$ entre os jatos 1 e 2 no decaimento ${ }^{7} p p \rightarrow Z^{\prime} \rightarrow\left(W^{+} \rightarrow\right.$

\footnotetext{
${ }^{5}$ Como no caso da distribuição do momento transverso do $Z^{\prime}$, a distribuição é aproximada, devido ao parton shower.

${ }^{6}$ Novamente, as distribuições são aproximadas.

${ }^{7}$ Obviamente, o restante dos constituintes dos prótons que não colidiram irão interagir para formar
} 
$\left.j_{1} j_{2}\right)\left(W^{-} \rightarrow j_{1}^{\prime} j_{2}^{\prime}\right)$ como definida na eq. (5.3).

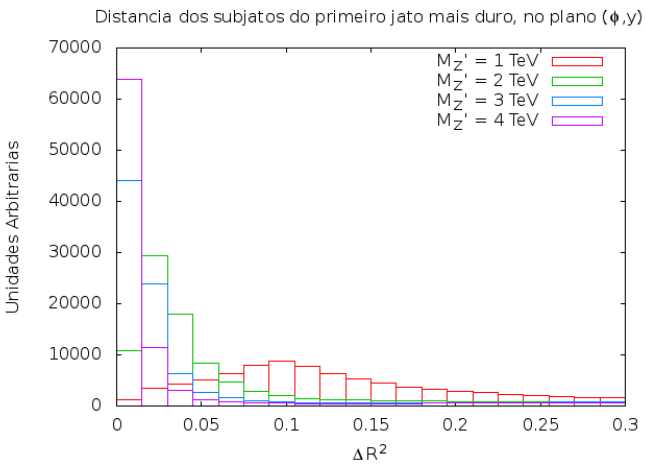

(a)

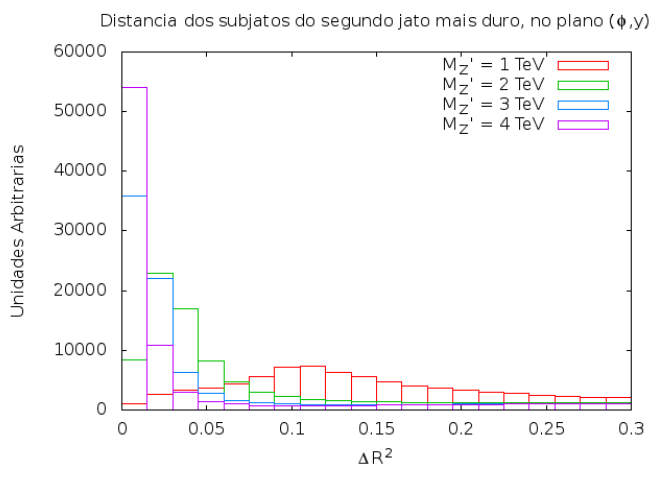

(b)

Figura 6.6: Distância $\Delta R^{2}$ entre os subjatos 1 e 2, produzidos no decaimento $W \rightarrow j_{1} j_{2}$ para diferentes valores da massa de $Z^{\prime}$. Os subjatos que constituem o jato mais duro do evento são mostrados na figura (a) e o segundo mais duro em (b). Pode-se ver que a medida que $M_{Z^{\prime}}$ aumenta, a distância entre os jatos fica cada vez menor (mais colimados) como se espera, dificultando muito a detecção dos jatos individualmente.

Vemos na figura 6.6 que para massas maiores do $Z^{\prime}$ e consequentemente maiores valores de $p_{t}$ dos $W^{\prime} s$, a distância entre os jatos vai caindo, fazendo com que a eficiência dos algoritmos de recombinação caia, [62]. Isso porque os jatos vão ficando cada vez mais próximos de maneira que eventualmente eles começam a se misturar e o algoritmo já não consegue mais distinguir um jato de outro. Em vez de identificar dois ou mais ${ }^{8}$ jatos leves, o algoritmo acha somente um jato gordo, sendo que a massa invariante pode ser próxima à massa do $W$, mesmo que seja um jato de QCD.

Por esse motivo torna-se essencial o uso de técnicas para analisar as subestruturas de um jato. Até aqui mostramos como o sinal se comporta para diferentes valores da massa do bóson $Z^{\prime}$. Podemos concluir que apesar de não pedir nenhum corte, os dois jatos mais duros dos eventos reconstroem muito bem a janela de massa do $W$ e a massa invariante dos dois jatos gordos reconstroem a massa do $Z^{\prime}$ usada na simulação. Ainda podemos observar o comportamento da distribuição da pseudorapidez dos dois jatos gordos. Espera-se que esses sejam centrais, para que não se perca o evento e para reduzir os efeitos de outras colisões moles. A figura 6.7 mostra essa distribuição.

singletos de cor, de maneira que a reação é na verdade: $p p \rightarrow Z^{\prime}+X$, com $X$ todo o restante do próton. Mas seguiremos a notação adotada na área de partículas, onde esse resto não é importante e portanto não o escreveremos.

${ }^{8}$ Devido à radiação, que pode dar origem a novos jatos 


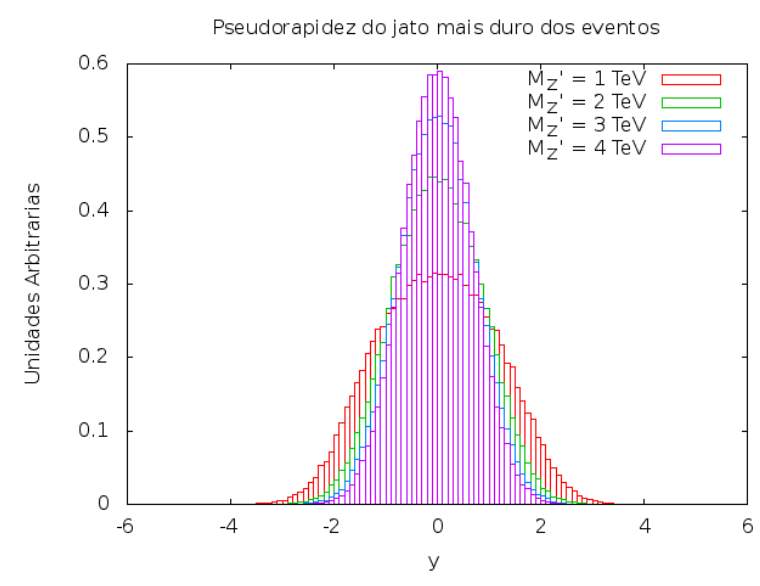

(a)

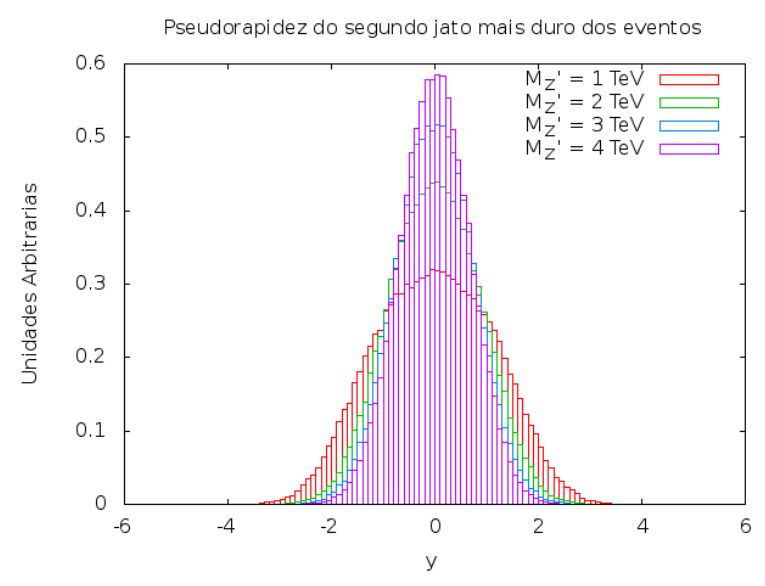

(b)

Figura 6.7: Distribuição da pseudorapidez do primeiro (a) e segundo (b) jatos mais duros nos eventos para diferentes massas de $Z^{\prime}$. A medida que $M_{Z^{\prime}}$ aumenta, as distribuições ficam mais estreitas, se concentrando na região central do detector.

Podemos ver que os jatos se encontram na região central do detector $(y<2.5)$ e a medida que a massa do $Z^{\prime}$ aumenta, os $W^{\prime} s$ se tornam mais centrais (como esperávamos pois seu $p_{t}$ aumenta). Caso os fundos apresentem um comportamento diferente, poderemos usar a pseudorapidez como um dos cortes.

Vemos que em todas as análises do sinal, o comportamento para diferentes massas do $Z^{\prime}$ é muito parecido, de maneira que a análise fica independente da massa do novo bóson ${ }^{9}$. Nosso próximo tópico é então descrever o modo como simulamos os eventos, os algoritmos utilizados para identificação dos jatos e como o fundo se comporta, para que possamos reduzí-lo.

\subsection{Pacotes, Fundos e Cortes}

Os eventos foram gerados e simulados usando o pacote MADGRAPH/MADEVENT [63] a nível partônico, esses eventos foram então transferidos para o pacote PYTHIA [30] para hadronização e parton showering. Temos então uma lista com todas as partículas geradas no evento produzidas durante a hadronização dos pártons. Usamos então o algoritmo $k_{t}$ para recombinar essas partículas em jatos através de rotinas do FastJet ([51] e [52]). $\mathrm{O}$ algoritmo $k_{t}$ foi escolhido devido à análise posterior das subestruturas dos jatos, como visto na secção 5.1.2, esse algoritmo possui uma ordem natural para voltarmos os passos da recombinação e rapidamente determinarmos os dois jatos principais originados do bóson $W$. O Fasjet foi escolhido para fazer o agrupamento, uma vez que trabalha com novas técnicas de recombinação para determinar os jatos vizinhos mais rapidamente, eliminando

\footnotetext{
${ }^{9}$ Pelo menos para massas da ordem de $1 \mathrm{TeV}$. Buscamos resultados que sejam independentes dos parâmetros utilizados no modelo (model independent), como a massa do $Z^{\prime}$, sua largura e seus acoplamentos com as partículas do MP.
} 
o problema do tempo de máquina crescer com $N^{3}$, como explicado em 5.1.2. Numa análise futura para melhor detalhar o processo pretende-se mudar o algoritmo de recombinação, principalmente para o $C / A$ para ver se há alteração nos resultados. $\mathrm{O}$ parâmetro $R$ adotado durante a recombinação foi o padrão, $R=0.7$.

$\mathrm{O}$ sinal gerado e analisado por esses pacotes foi $p p \rightarrow Z^{\prime} \rightarrow\left(W^{+} \rightarrow j j\right)\left(W^{-} \rightarrow j j\right)$ sendo que os jatos provenientes dos $W^{\prime} s$ estão suficientemente próximos, de maneira que o algoritmo de recombinação sozinho não consegue distinguir os dois jatos leves, determinando assim somente dois jatos gordos.

Devido a presença de jatos no estado final do nosso sinal, existem muitos processos no MP que podem dar origem ao mesmo tipo de assinatura no LHC. Entre eles temos:

- $p p \rightarrow j j$, com a massa invariante de cada jato na janela de massa do $W$. O background de QCD pode não parecer um grande problema, pois serão poucos os eventos nos quais a massa invariante de um jato de QCD se confunda com um $W$. Porém a secção de choque deste processo é enorme, de maneira que se observa um número grande de jatos que satisfazem tal critério;

- $p p \rightarrow\left(W^{+} \rightarrow j j\right)\left(W^{-} \rightarrow j j\right)$, este representa um grande problema, pois tem exatamente a mesma assinatura do sinal, porém com os $W^{\prime} s$ sendo formados por partículas conhecidas no MP, como o fóton, o $Z$ ou o Higgs. Nosso algoritmo deve ser capaz de identificar os $W^{\prime} s$ de um processo que veio de um novo bóson vetorial e excluir $W^{\prime} s$ provenientes de processos do MP;

- $p p \rightarrow(Z \rightarrow j j)(Z \rightarrow j j)$, com os bósons $Z$ sendo confundidos com $W$, o que não é raro, uma vez que a janela de massa dessas partículas é parecida $\left(M_{Z}-M_{W} \approx\right.$ $10 \mathrm{GeV})$.

- $p p \rightarrow\left(t \rightarrow b\left(W^{+} \rightarrow j j\right)\right)\left(\bar{t} \rightarrow \bar{b}\left(W^{-} \rightarrow j j\right)\right)$, este também representa um potencial problema, pois existem dois $W^{\prime} s$ no processo. Para $b^{\prime} s$ suficientemente afastados, este processo também tem o mesmo rastro do sinal no detector;

- $p p \rightarrow j\left(W^{ \pm} \rightarrow j j\right)$, com um jato de QCD sendo confundido com um $W$;

- $p p \rightarrow j(Z \rightarrow j j)$ com um jato de QCD sendo confundido com um $W$ e o jato gordo (jato do $Z$ ) também sendo confundido com um $W$;

- $p p \rightarrow Z^{\prime} \rightarrow j j$, esse processo é considerado como sinal, uma vez que é de fato um $Z^{\prime}$ que deu origem aos jatos. Aqui os jatos de QCD são confundidos com jatos pesados como em $p p \rightarrow j j$ no MP;

- $p p \rightarrow Z^{\prime} \rightarrow\left(t \rightarrow b\left(W^{+} \rightarrow j j\right)\right)\left(\bar{t} \rightarrow \bar{b}\left(W^{-} \rightarrow j j\right)\right)$, esse também é um processo que consideramos como sinal devido ao $Z^{\prime}$ intermediário. Sua assinatura é como a do par $t \bar{t}$ do MP. 
Como vimos na figura 6.3, os $W^{\prime} s$ produzidos pelo novo bóson estão bastante rápidos, enquanto que os jatos produzidos pelos fundos do MP normalmente possuem $p_{t}$ muito menor. Fez-se então uma análise dos backgrounds apresentados acima para que se pudesse conhecer melhor seus comportamentos. Para que se possa comparar a eficiência de identificação do nosso algoritmo entre o sinal e os fundos, é necessário que a geração de eventos para o fundo ocorra na região de interesse. Tomemos o fundo de QCD como exemplo, a seç̧ão de choque do processo $p p \rightarrow j j \operatorname{com} p_{t}^{j}>20 \mathrm{GeV}$ é da ordem de $10^{8} \mathrm{pb}$ o que é muito maior que a secção de choque do nosso sinal. Porém o grosso desses eventos são gerados para valores de $p_{t}$ muito baixos, que não irão reconstruir um $Z^{\prime}$ com massa na ordem de $1 \mathrm{TeV}$, como podemos ver na figura 6.8 onde simulamos o fundo de QCD com o corte padrão ${ }^{10}$ do LHC. Assim, analisamos a região de $p_{t}$ onde os backgrounds começam a reconstruir corretamente a massa da ressonância para que se possa pedir um corte no momento transverso mínimo no estágio de geração de eventos.

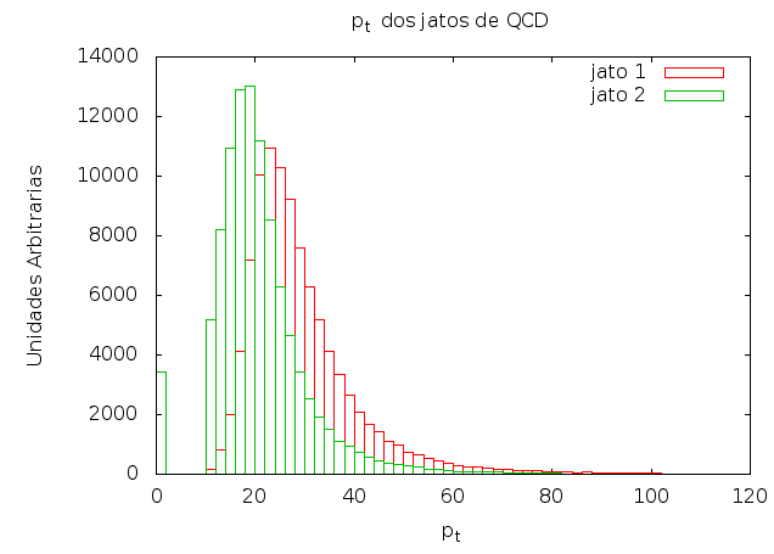

Figura 6.8: Distribuição do $p_{t}$ dos dois jatos mais duros nos eventos $p p \rightarrow j j$ com os eventos gerados com momento transverso mínimo: $p_{t}^{\text {min }}=20 \mathrm{GeV}$. Vê-se que a maior parte dos eventos são gerados com $p_{t}$ baixos que não irão reconstruir massas da ordem de $1 \mathrm{TeV}$.

Pode-se ver na figura 6.8 que o grosso da secção de choque ocorre para $p_{t}$ baixos, de maneira que precisamos gerar os eventos com um valor de $p_{t}$ maior ${ }^{11}$ para que se possa reconstruir massas da ordem de $1 \mathrm{TeV}$ e assim determinar a região onde os fundos podem ser confundidos com nosso sinal. Analisamos então os casos com dois jatos de QCD $(p p \rightarrow j j)$, um jato de QCD e um bóson eletrofraco $\left(p p \rightarrow j W^{+}\right.$que é análogo ao caso $j W^{-}$e $j Z$ ) e dois bósons eletrofracos $\left(p p \rightarrow W^{+} W^{-}\right.$análogo ao processo $\left.Z Z\right)$, sendo que os bósons sempre decaem hadronicamente. Analisamos ainda a produção dos

\footnotetext{
${ }^{10}$ Todos os jatos detectados pelo LHC devem ter no mínimo $p_{t}=20 \mathrm{GeV}$ para que o evento seja guardado para análise. Isso porque os eventos de interesse ocorrem para altos valores de momento transverso e esse corte já elimina grande parte do fundo de QCD que muitas vezes é colinear com o feixe de prótons.

${ }^{11}$ Isso fará com que a secção de choque dos fundos diminua o suficiente para tornar a análise viável.
} 
pares $t \bar{t}$ decaindo hadronicamente. Assim obtivémos as regiões de interesse para o $p_{t}$ onde o algoritmo terá mais dificuldade de diferenciar sinal de fundo. As figuras 6.9, 6.10, 6.11 e 6.12 mostram o $p_{t}$ dos dois jatos mais duros dos eventos e a massa invariante da soma desses jatos. Os títulos das figuras correspondem ao corte aplicado no momento transverso dos jatos na geração dos eventos, ou seja, cada $j$ do estado final foi gerado com $p_{t}^{\text {min }}$ indicado na figura.
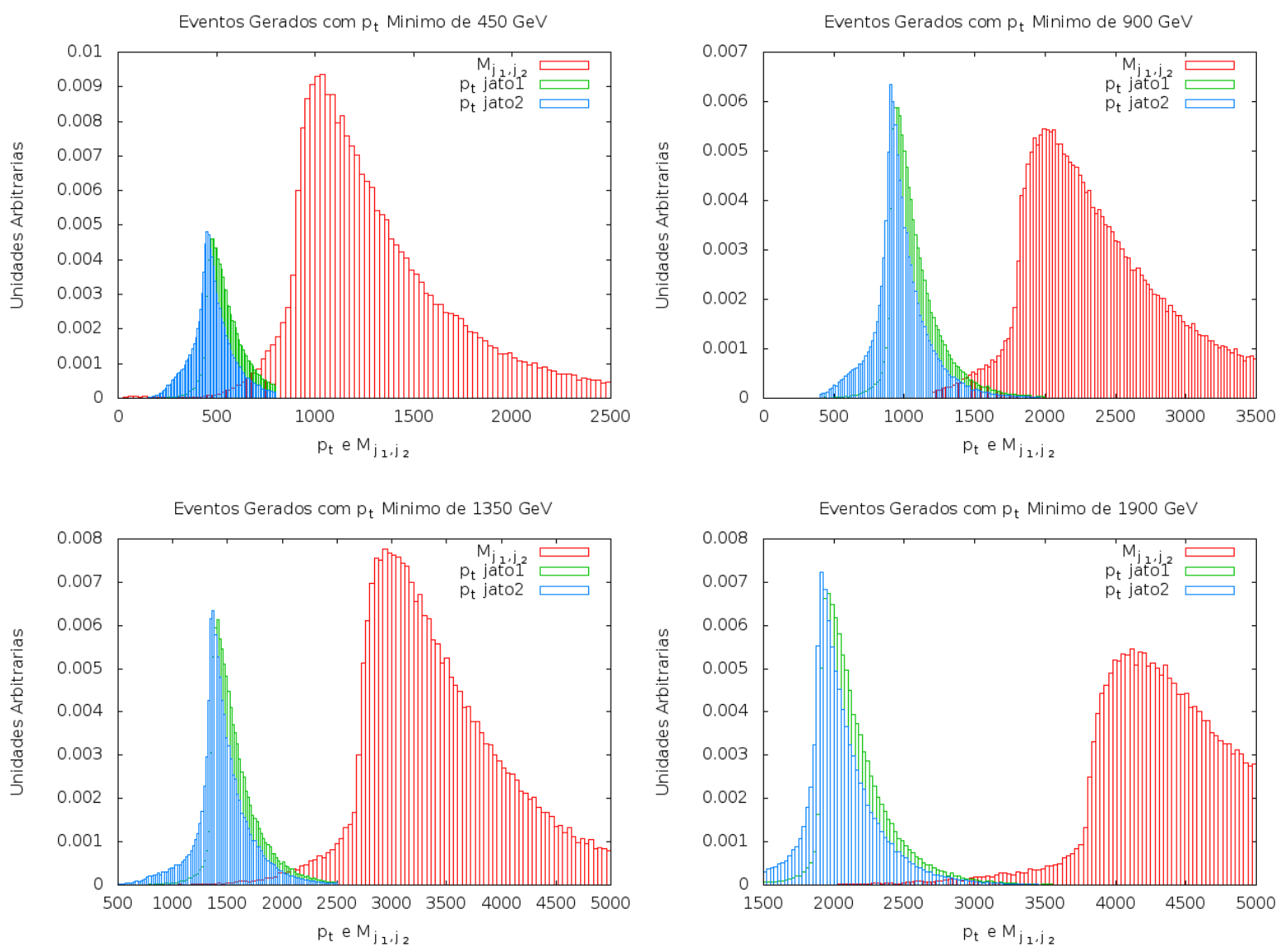

Figura 6.9: Distribuição do momento transverso dos dois jatos mais duros nos eventos $p p \rightarrow j_{1} j_{2}$ e a Massa Invariante da recombinação dos dois jatos duros. Em cada figura está representado o $p_{t}^{\text {min }}$ usado na geração dos eventos para que se pudesse reconstruir a massa invariante desejada. 

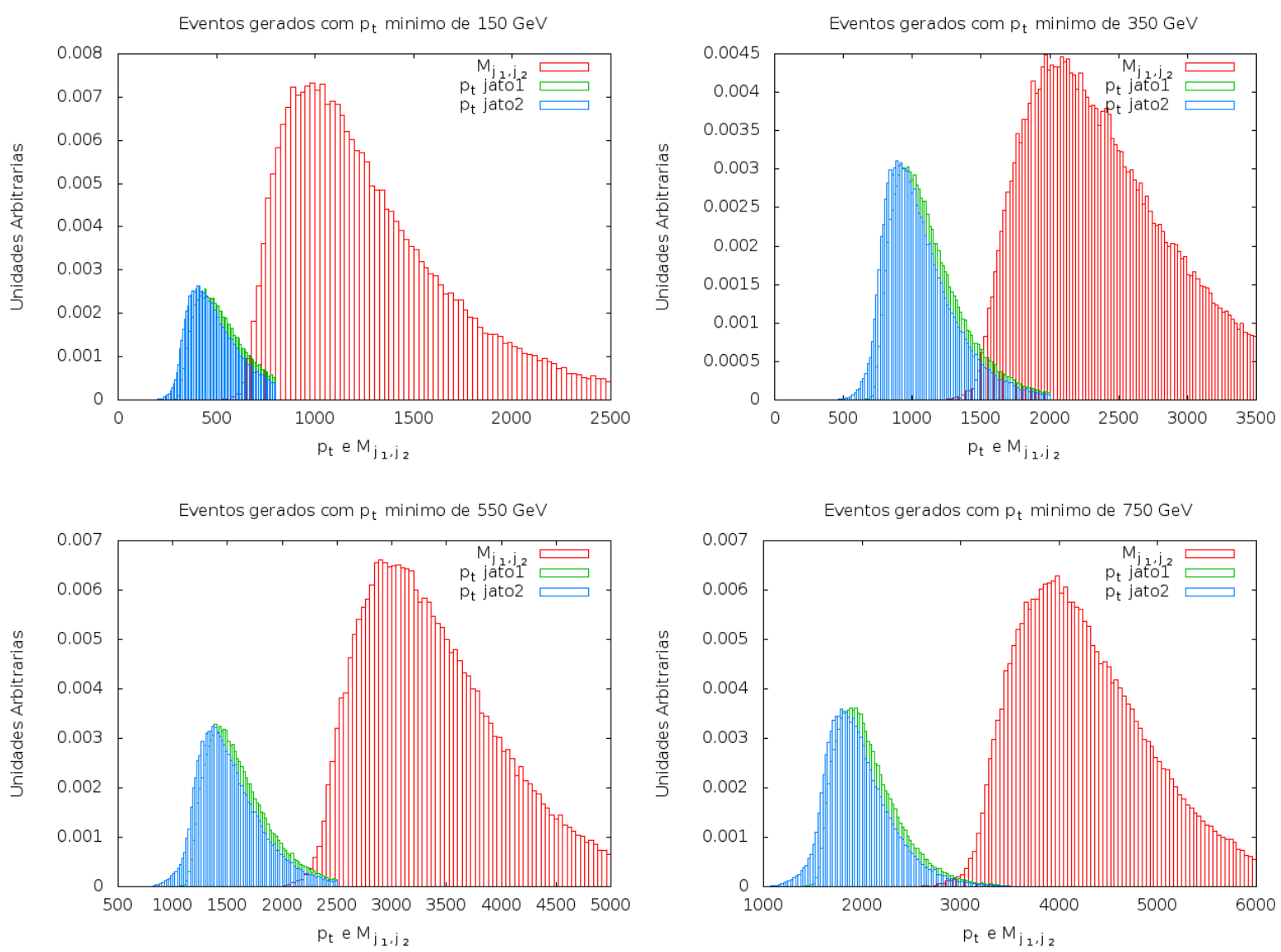

Figura 6.10: Distribuição do momento transverso dos dois jatos mais duros nos eventos $p p \rightarrow W^{+} W^{-} \rightarrow j j j j$ e a Massa Invariante da recombinação dos dois jatos duros. Em cada figura está representado o $p_{t}^{\min }$ usado na geração dos eventos para que se pudesse reconstruir a massa invariante desejada. 

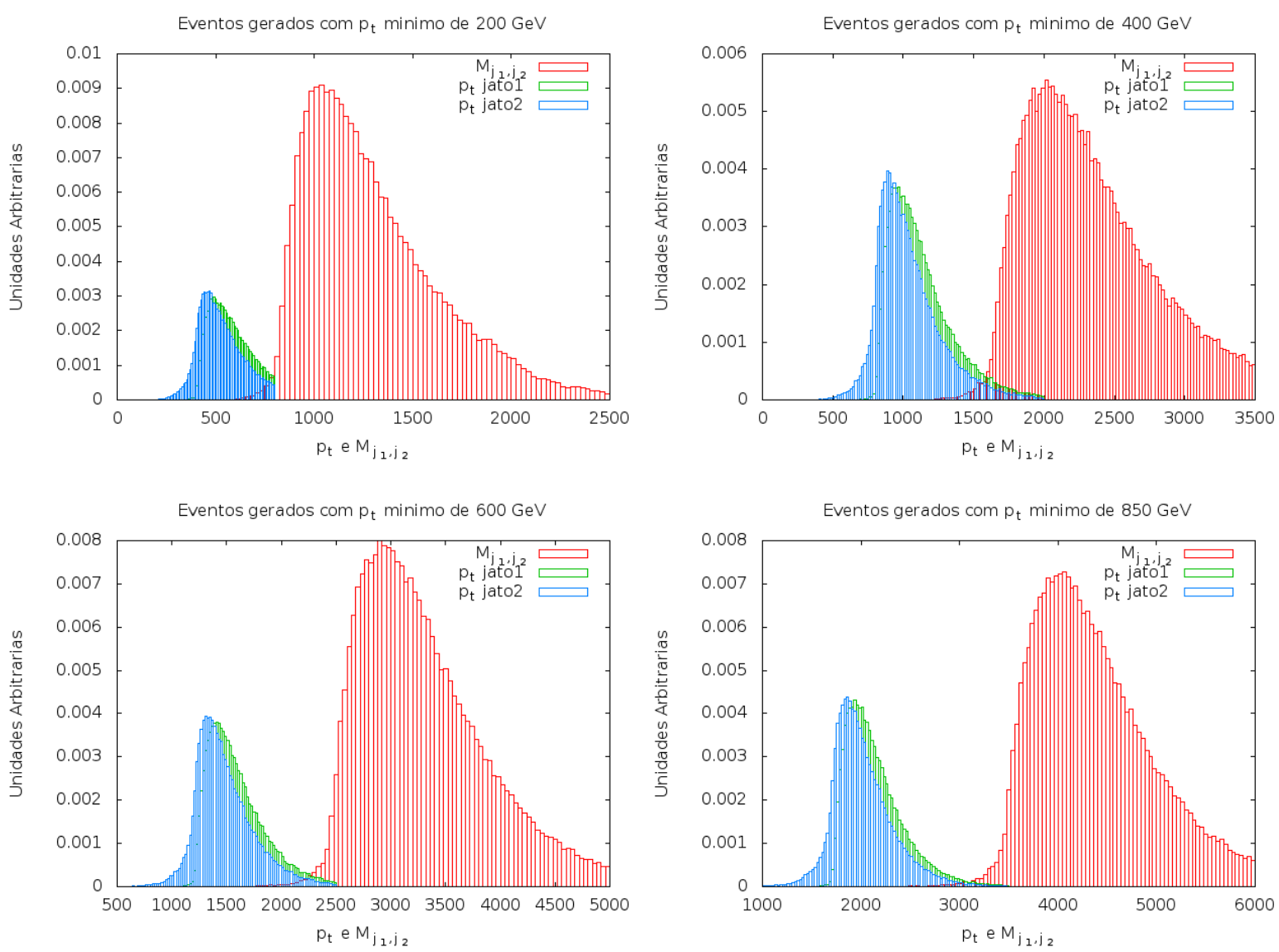

Figura 6.11: Distribuição do momento transverso dos dois jatos mais duros nos eventos $p p \rightarrow j_{1} W^{+} \rightarrow j_{1} j j$ e a Massa Invariante da recombinação dos dois jatos duros. Em cada figura está representado o $p_{t}^{\text {min }}$ usado na geração dos eventos para que se pudesse reconstruir a massa invariante desejada. 

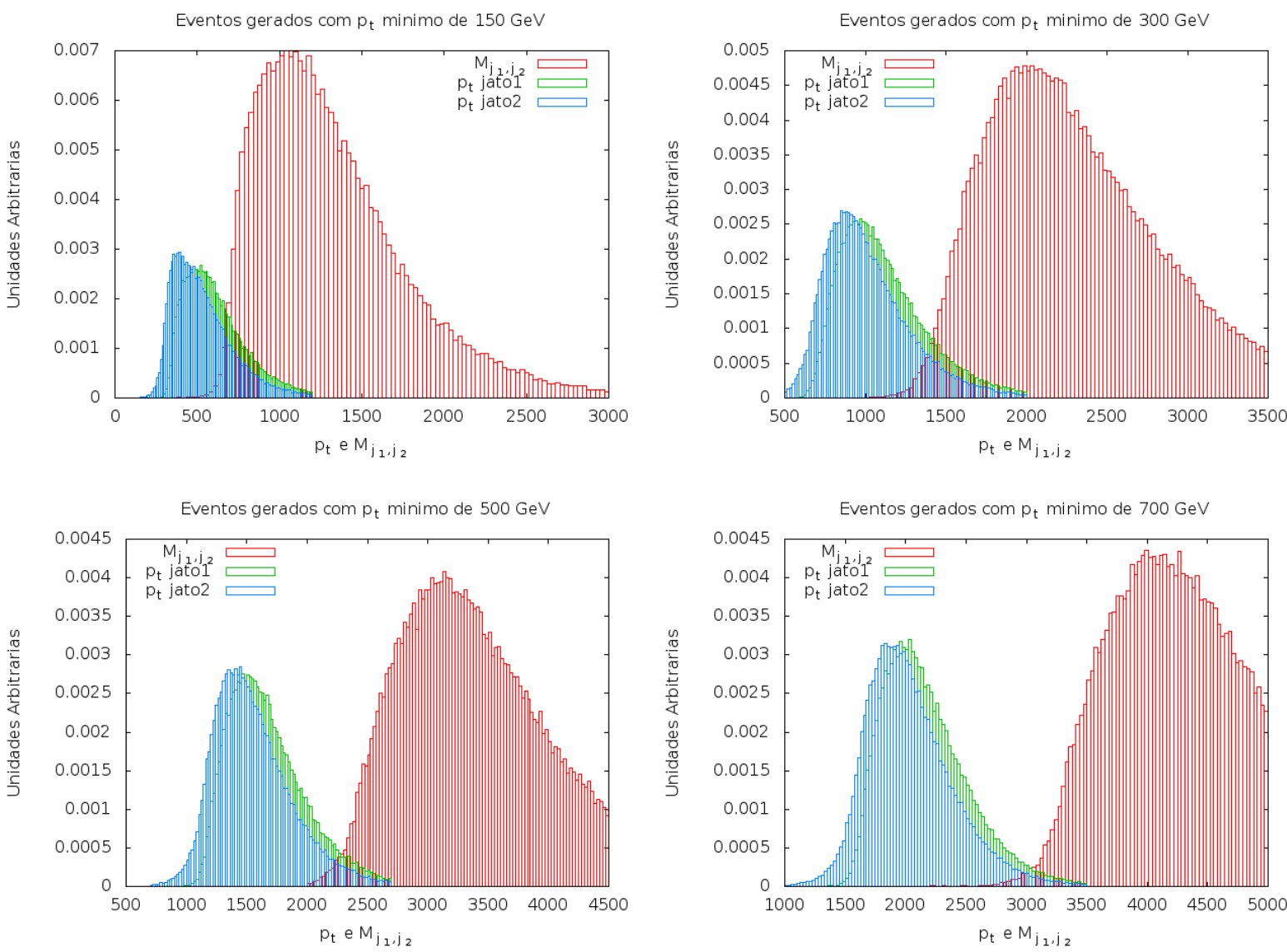

Figura 6.12: Distribuição do momento transverso dos dois jatos mais duros nos eventos $p p \rightarrow t \bar{t} \rightarrow b \bar{b} j j j j$ e a Massa Invariante da recombinação dos dois jatos duros. Em cada figura está representado o $p_{t}^{\text {min }}$ usado na geração dos eventos para que se pudesse reconstruir a massa invariante desejada.

Na figura 6.13 mostramos alguns cortes em eventos diferentes, só para ilustrar que a troca do bóson vetorial não muda o valor dos cortes, uma vez que o comportamento é o mesmo. 


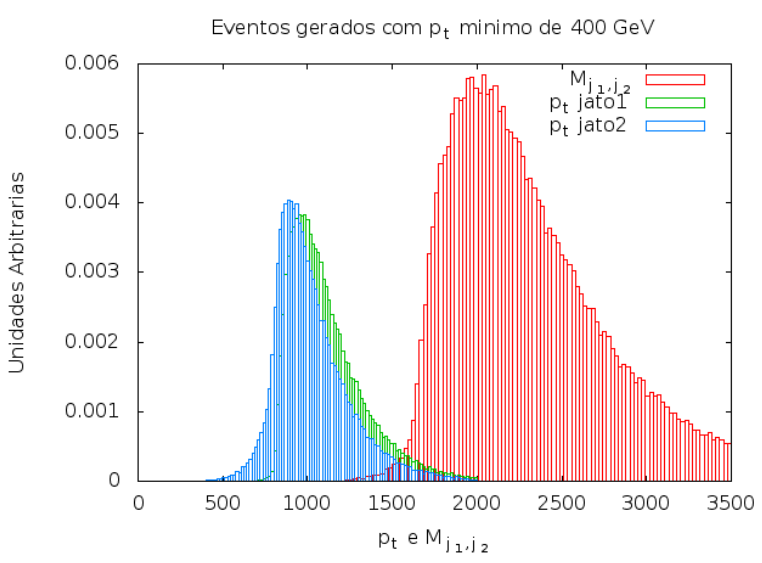

(a)

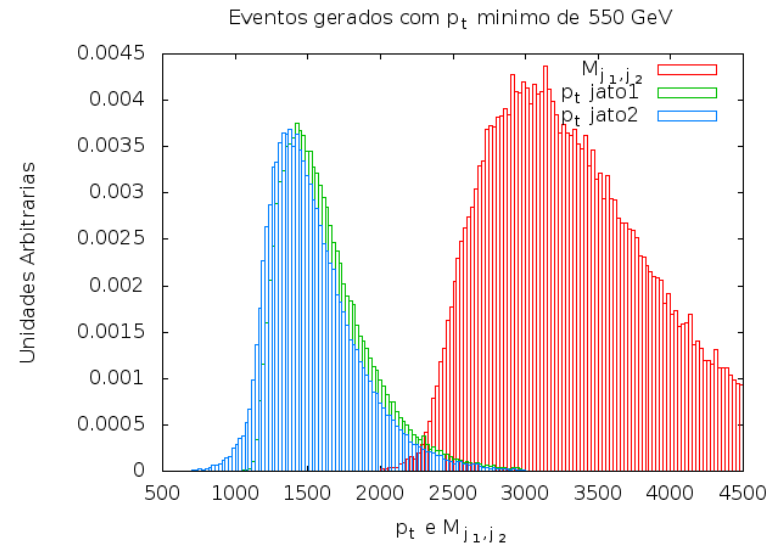

(b)

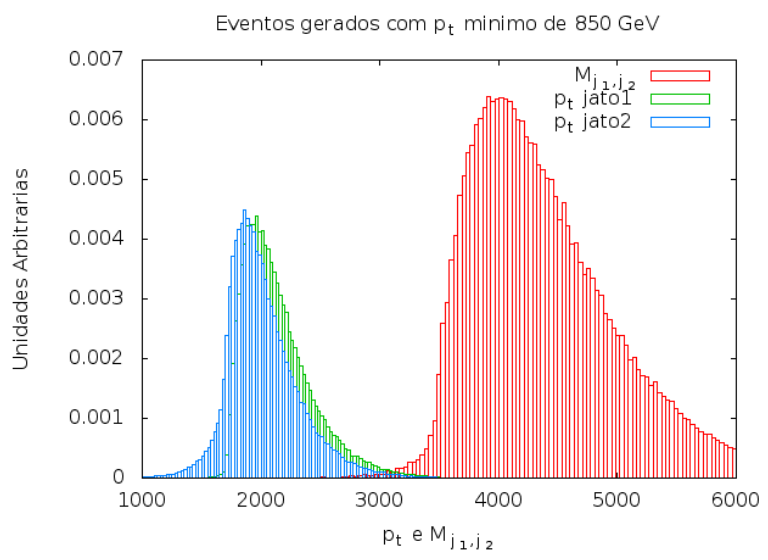

(c)

Figura 6.13: Distribuição do momento transverso dos dois jatos mais duros nos eventos e a Massa Invariante da recombinação dos dois jatos duros. Em cada figura está representado o $p_{t}^{\text {min }}$ usado na geração dos eventos para que se pudesse reconstruir a massa invariante desejada. Os eventos são: (a) $p p \rightarrow j_{1} W^{-} \rightarrow j_{1} j j$ para $2 \mathrm{TeV}$; (b) $p p \rightarrow Z Z \rightarrow j j j j$ para $3 \mathrm{TeV}$; e (c) $p p \rightarrow j_{1} Z \rightarrow j_{1} j j$ para $4 \mathrm{TeV}$.

Dessa forma, para que se possa obter eventos na região de interesse que reconstrói a massa do $Z^{\prime}$, os cortes no momento transverso dos jatos de QCD são os da tabela 6.1.

\begin{tabular}{|c|c|}
\hline Massa Invariante & $p_{t}^{\min }(\mathrm{GeV})$ \\
\hline \hline $1 \mathrm{TeV}$ & 450 \\
\hline $2 \mathrm{TeV}$ & 900 \\
\hline $3 \mathrm{TeV}$ & 1350 \\
\hline $4 \mathrm{TeV}$ & 1900 \\
\hline
\end{tabular}

Tabela 6.1: Corte no $p_{t}$ dos jatos no processo $p p \rightarrow j j$ para que a geração de eventos ocorra na região de interesse.

Para os eventos com dois bósons eletrofracos decaindo hadrônicamente ( $W W$ e $Z Z)$, os cortes são os da tabela 6.2 . 


\begin{tabular}{|c|c|}
\hline Massa Invariante & $p_{t}^{\min }(\mathrm{GeV})$ \\
\hline \hline $1 \mathrm{TeV}$ & 150 \\
\hline $2 \mathrm{TeV}$ & 350 \\
\hline $3 \mathrm{TeV}$ & 550 \\
\hline $4 \mathrm{TeV}$ & 750 \\
\hline
\end{tabular}

Tabela 6.2: Corte no $p_{t}$ dos jatos no processo $p p \rightarrow V V \rightarrow j j j j$, onde $V=W, Z$ para que a geração de eventos ocorra na região de interesse.

Os eventos com um jato de QCD e um bóson vetorial decaindo hadrônicamente estão na tabela 6.3.

\begin{tabular}{|c|c|}
\hline Massa Invariante & $p_{t}^{\text {min }}(\mathrm{GeV})$ \\
\hline \hline $1 \mathrm{TeV}$ & 200 \\
\hline $2 \mathrm{TeV}$ & 400 \\
\hline $3 \mathrm{TeV}$ & 600 \\
\hline $4 \mathrm{TeV}$ & 850 \\
\hline
\end{tabular}

Tabela 6.3: Corte no $p_{t}$ dos jatos no processo $p p \rightarrow j_{1} V \rightarrow j_{1} j j$, onde $V=W, Z$ para que a geração de eventos ocorra na região de interesse.

E finalmente para os eventos com pares $t \bar{t}$ decaindo hadronicamente, os cortes estão na tabela 6.4.

\begin{tabular}{|c|c|}
\hline Massa Invariante & $p_{t}^{\text {min }}(\mathrm{GeV})$ \\
\hline \hline $1 \mathrm{TeV}$ & 150 \\
\hline $2 \mathrm{TeV}$ & 300 \\
\hline $3 \mathrm{TeV}$ & 500 \\
\hline $4 \mathrm{TeV}$ & 700 \\
\hline
\end{tabular}

Tabela 6.4: Corte no $p_{t}$ dos jatos no processo $p p \rightarrow t \bar{t} \rightarrow b \bar{b} j j j j$, para que a geração de eventos ocorra na região de interesse.

Com isso em mente e lembrando que somente o algoritmo de recombinação não será de grande ajuda para vermos o $Z^{\prime}$, há necessidade de observarmos subestrutura nos jatos recombinados. Para tanto utilizamos o seguinte procedimento, baseado na técnica de queda de massa apresentada na secção 5.1.3, nos dois jatos mais duros do evento:

1. Recombina-se todas as partículas após a hadronização em jatos de momento transversos decrescentes, de maneira que o primeiro jato é o jato mais duro do evento;

2. De todos os jatos recombinados pelo algoritmo no passo 1, guarda-se somente os que satisfazem:

$$
p_{t}^{j a t o} \geq p_{t}^{\text {min }}
$$

sendo $p_{t}^{\text {min }}$ o momento transverso mínimo para que o jato seja um candidato a um $W$ e a escolha depende da massa do $Z^{\prime}$. A tabela 6.5 mostra os valores de $p_{t}^{\text {min }}$ utilizados. 
3. Dado um jato $j$, define-se $j_{1}$ e $j_{2}$ os dois proto-jatos que foram recombinados ${ }^{12}$ no jato $j$, essencialmente são os dois proto-jatos do último passo do algoritmo de recombinação, sendo $m_{j_{1}} \geq m_{j_{2}}$;

4. Se $m_{j_{1}}<\mu m_{j}$ e $\min \left(p_{T, j_{1}}^{2}, p_{T, j_{2}}^{2}\right) \Delta R_{j_{1}, j_{2}}^{2}>y^{2} m_{j}^{2}$ e $\left|m_{j}-M_{W}\right|<\Delta M_{W}$ o jato $j$ é identificado como sendo um bóson $W$, se $j$ não satisfaz todas as condições simultaneamente, então redefine-se $j_{1}$ como $j$ e aplica-se novamente o algoritmo. Caso nenhum dos proto-jatos satisfaça tais critérios o jato não é dito ser um $W$ e o evento não é classificado como sinal;

5. Se os dois jatos mais duros do evento forem identificados como $W^{\prime} s$ e a massa invariante dos dois jatos recombinados estiverem na janela de massa do $Z^{\prime}$, ou seja $\left|m_{\left(j_{1}+j_{2}\right)}-M_{Z^{\prime}}\right|<\frac{M_{Z^{\prime}}}{10}$, então o evento é identificado como sinal!

A janela de massa do $W$ e do $Z^{\prime}$ dependem das larguras dessas partículas. Para o $W$ usamos $\Delta M_{W}=15 \mathrm{GeV}$, de modo que a janela de massa fica

$$
65 \mathrm{GeV} \leq m_{j} \leq 95 \mathrm{GeV}
$$

e para o $Z^{\prime}$ usamos $\Gamma_{Z^{\prime}}=\frac{M_{Z^{\prime}}}{10}$. Este valor de largura faz com que o sinal seja mais facilmente $^{13}$ visto, já que é uma largura estreita.

A relação $\min \left(p_{T, j_{1}}^{2}, p_{T, j_{2}}^{2}\right) \Delta R_{j_{1}, j_{2}}^{2}$ é a distância $k_{t}$ entre os proto-jatos $j_{1}$ e $j_{2}$ com $\Delta R^{2}=\left(\phi_{j_{1}}-\phi_{j_{2}}\right)^{2}+\left(y_{j_{1}}-y_{j_{2}}\right)^{2}$ como definido em 5.1.2. Os parâmetros $\mu$ e $y$ são ajustáveis e foram usados para otimizar a identificação do sinal, minimizando a má-identificação dos fundos (mistagging). A figura 6.14 mostra as distribuições dos parâmetros $\mu$ e $y^{2}$ para diferentes eventos: o sinal $Z^{\prime} \rightarrow W W$ e os fundos $j j, j W W W$ e $t \bar{t}$, assim podemos comparar as curvas para escolhermos um valor otimizado desses parâmetros.

\footnotetext{
${ }^{12}$ Usualmente os jatos $j_{1}$ e $j_{2}$ são chamados de pais do jato $j$.

${ }^{13}$ Para tornar a análise ainda mais independente de parâmetros é interessante estudar diferentes valores para a largura do $Z^{\prime}$. Isso não foi feito nesta dissertação, mas é imediato implementar nas simulações e pretendemos continuar em trabalhos futuros.
} 


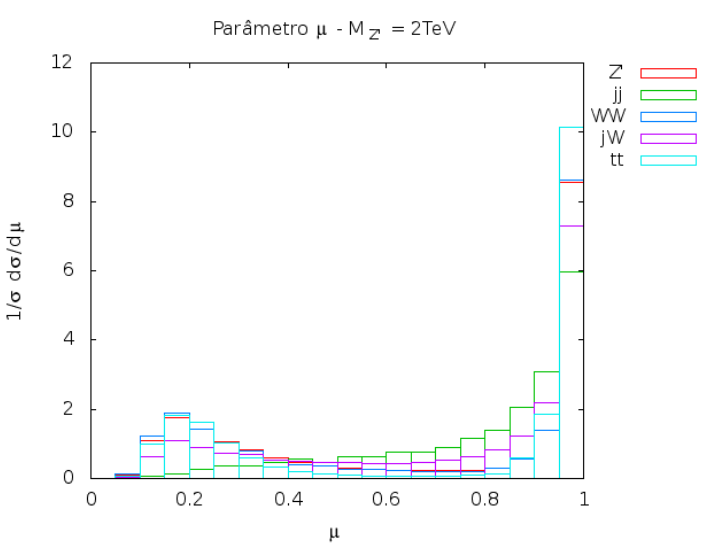

(a)

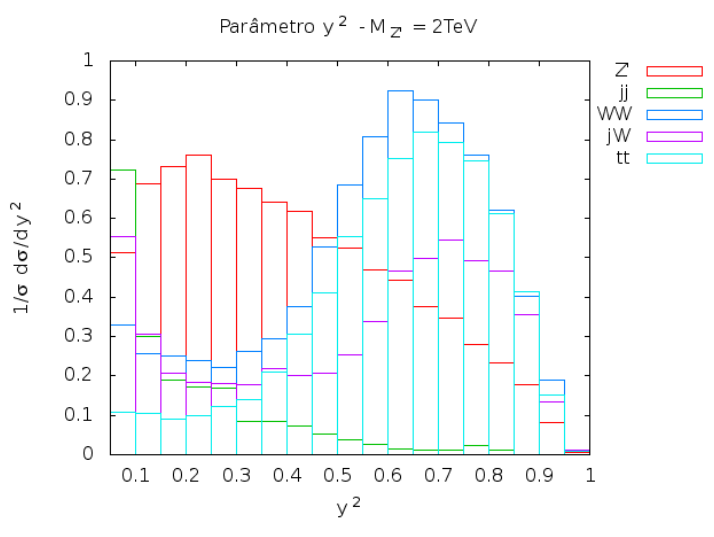

(b)

Figura 6.14: Parâmetros $\mu$ (a) e $y^{2}$ (b) do sinal: $Z^{\prime} \rightarrow W W$ e os processos do MP: $p p \rightarrow j j, j W, W W$ e $t \bar{t}$ para $M_{Z^{\prime}}=2 \mathrm{TeV}$.

\begin{tabular}{|c|c|c|c|c|}
\hline & $M_{Z^{\prime}}=1 \mathrm{TeV}$ & $M_{Z^{\prime}}=2 \mathrm{TeV}$ & $M_{Z^{\prime}}=3 \mathrm{TeV}$ & $M_{Z^{\prime}}=4 \mathrm{TeV}$ \\
\hline \hline$p_{t}^{\text {min }}$ & $300 \mathrm{GeV}$ & $700 \mathrm{GeV}$ & $1000 \mathrm{GeV}$ & $1500 \mathrm{GeV}$ \\
\hline
\end{tabular}

Tabela 6.5: Momento transverso mínimo dos jatos para que sejam candidatos a um jato de $W$ para diferentes massas do $Z^{\prime}$.

Analisando as figuras 6.14 (a) e (b), adotamos os valores $\mu=0.4$ e $y^{2}=0.1$. Então em todos os eventos identificados como um $W$ há uma queda de massa de mais de 60\%, ou seja, o proto-jato mais massivo deve ter no máximo $40 \%$ da massa do jato. Isso em geral não ocorre para jatos de QCD, pois na maior parte dos casos um dos proto-jatos é um glúon de radiação e então o proto-jato mais massivo tem uma massa mais perto do jato, escolhendo então os eventos com $\mu<0.4$ conseguimos diminuir muito o fundo de QCD. A figura 6.15 mostra o parâmetro $\mu$ usando a escala logaritmica e deixa esse aspecto dos jatos de QCD mais aparente. Para o parâmetro $y^{2}$ a figura 6.16 também está em escala logaritmica, pois na figura 6.14 (b) mostramos somente os valores $y^{2} \geq 0.05$ para melhor visualização do comportamento desse parâmetro. Há um pico entre 0 e 0.1 que é dominado pelos jatos de QCD, uma vez que a distância $k_{t}$ entre os proto-jatos é pequena, pois o $p_{t}$ da radiação é baixo (mole) e ela é colinear $\left(\Delta R^{2}\right.$ pequeno). Soma-se isso ao fato de que a distância- $k_{t}$ é dividida pela massa do jato gordo que está na janela de massa do $W$ fazendo com que a distribuição de $y^{2}$ nos jatos de QCD caia rapidamente e por isso escolhemos os eventos com $y^{2}>0.1$. 


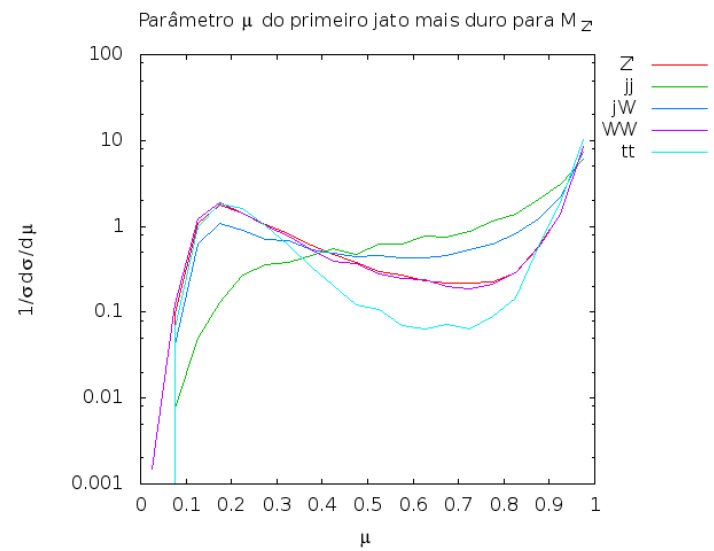

(a)

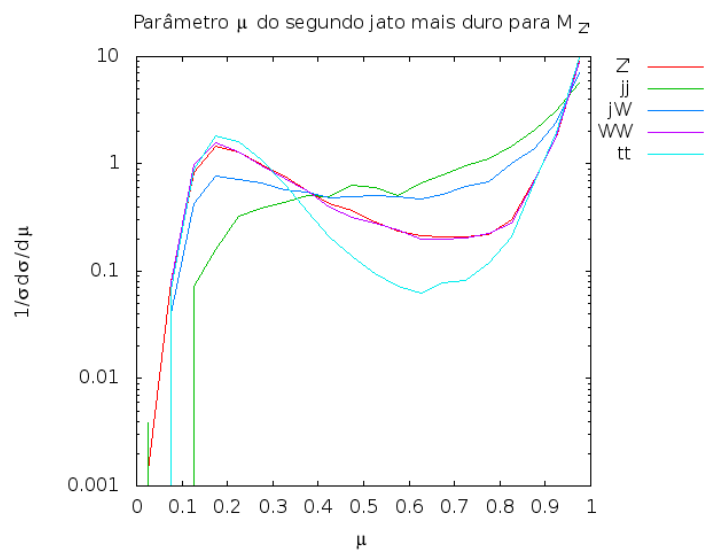

(b)

Figura 6.15: Parâmetro $\mu$ em escala logaritmica do primeiro (a) e segundo (b) jatos mais duros dos eventos para $M_{Z^{\prime}}=2 \mathrm{TeV}$.

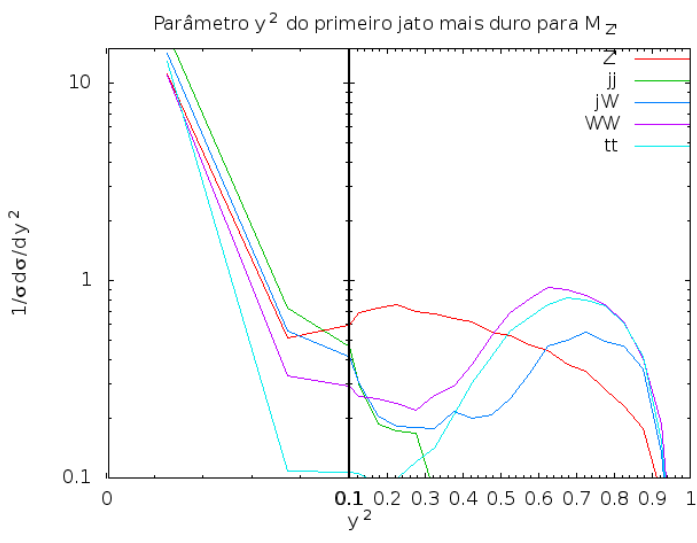

(a)

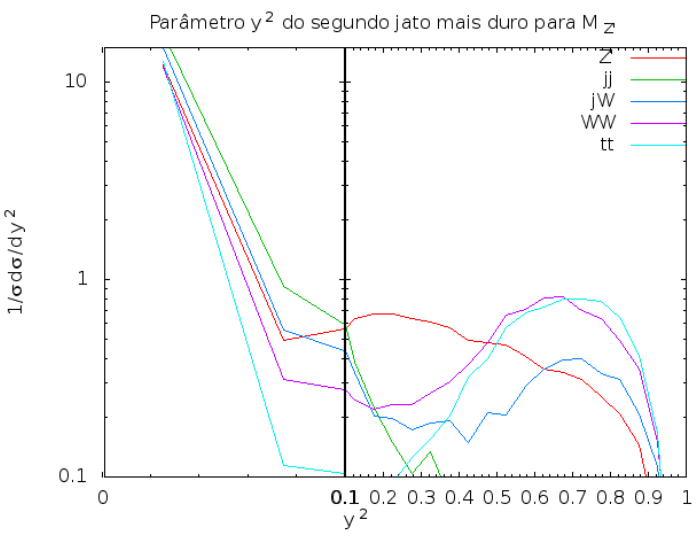

(b)

Figura 6.16: Parâmetro $y^{2}$ em escala logaritmica do primeiro (a) e segundo (b) jatos mais duros dos eventos para $M_{Z^{\prime}}=2 \mathrm{TeV}$. Nas figuras há um aumento na região entre 0 e 0.1 para explicitar o comportamento dos jatos de QCD nessa região.

Finalmente, utilizamos esse procedimento para simular nossos eventos. A análise foi feita para a $M_{Z^{\prime}}=1,2,3,4 \mathrm{TeV}$. As tabelas $6.6,6.7,6.8$ e 6.9 mostram a eficiência $(\epsilon)$ do algoritmo em se identificar o sinal e também a má identificação (mistagging) dos fundos, a secção de choque $\sigma$ de cada processo (lembrando que os cortes das tabelas $6.1,6.2,6.3$ e 6.4 foram aplicados já na geração dos eventos) e ainda o produto $\epsilon \cdot \sigma$. 


\begin{tabular}{|c|c|c|c|}
\hline Processo & Eficiência, $\epsilon$ & Secção de Choque, $\sigma(\mathrm{pb})$ & $\epsilon \cdot \sigma$ \\
\hline \hline$Z^{\prime} \rightarrow W W$ & 0.33 & $3.75 \cdot 10^{+1}$ & $1.25 \cdot 10^{+1}$ \\
\hline$Z^{\prime} \rightarrow j j$ & $6.00 \cdot 10^{-4}$ & $6.13 \cdot 10^{-1}$ & $1.02 \cdot 10^{+3}$ \\
\hline$Z^{\prime} \rightarrow t \bar{t}$ & $1.87 \cdot 10^{-2}$ & $1.08 \cdot 10^{+2}$ & 2.02 \\
\hline$W W$ & 0.12 & $3.76 \cdot 10^{-3}$ & $4.63 \cdot 10^{-4}$ \\
\hline$Z Z$ & 0.11 & $4.92 \cdot 10^{-4}$ & $5.24 \cdot 10^{-5}$ \\
\hline$j j$ & $7.90 \cdot 10^{-4}$ & $1.01 \cdot 10^{+3}$ & $8.00 \cdot 10^{-1}$ \\
\hline$t \bar{t}$ & 0.15 & $6.08 \cdot 10^{-2}$ & $9.28 \cdot 10^{-3}$ \\
\hline$j W^{+}$ & $8.49 \cdot 10^{-3}$ & $4.80 \cdot 10^{-1}$ & $4.07 \cdot 10^{-3}$ \\
\hline$j W^{-}$ & $8.37 \cdot 10^{-3}$ & $2.35 \cdot 10^{-1}$ & $1.97 \cdot 10^{-3}$ \\
\hline$j Z$ & $8.21 \cdot 10^{-3}$ & $2.38 \cdot 10^{-1}$ & $1.96 \cdot 10^{-3}$ \\
\hline
\end{tabular}

Tabela 6.6: Eficiência e secção de choque para $M_{Z^{\prime}}=1 \mathrm{TeV}$.

\begin{tabular}{|c|c|c|c|}
\hline Processo & Eficiência, $\epsilon$ & Secção de Choque, $\sigma(\mathrm{pb})$ & $\epsilon \cdot \sigma$ \\
\hline \hline$Z^{\prime} \rightarrow W W$ & 0.32 & 7.37 & 2.39 \\
\hline$Z^{\prime} \rightarrow j j$ & $3.20 \cdot 10^{-4}$ & $5.21 \cdot 10^{+1}$ & $1.67 \cdot 10^{-2}$ \\
\hline$Z^{\prime} \rightarrow t \bar{t}$ & $8.22 \cdot 10^{-3}$ & $1.03 \cdot 10^{+1}$ & $8.42 \cdot 10^{-2}$ \\
\hline$W W$ & 0.16 & $5.65 \cdot 10^{-5}$ & $9.02 \cdot 10^{-6}$ \\
\hline$Z Z$ & 0.14 & $7.19 \cdot 10^{-6}$ & $1.01 \cdot 10^{-6}$ \\
\hline$j j$ & $6.80 \cdot 10^{-4}$ & $2.20 \cdot 10^{+1}$ & $1.50 \cdot 10^{-2}$ \\
\hline$t \bar{t}$ & 0.22 & $3.55 \cdot 10^{-4}$ & $7.69 \cdot 10^{-5}$ \\
\hline$j W^{+}$ & $1.15 \cdot 10^{-2}$ & $1.21 \cdot 10^{-2}$ & $1.40 \cdot 10^{-4}$ \\
\hline$j W^{-}$ & $1.11 \cdot 10^{-2}$ & $4.74 \cdot 10^{-3}$ & $5.27 \cdot 10^{-5}$ \\
\hline$j Z$ & $1.10 \cdot 10^{-2}$ & $5.45 \cdot 10^{-3}$ & $5.97 \cdot 10^{-5}$ \\
\hline
\end{tabular}

Tabela 6.7: Eficiência e secção de choque para $M_{Z^{\prime}}=2 \mathrm{TeV}$.

\begin{tabular}{|c|c|c|c|}
\hline Processo & Eficiência, $\epsilon$ & Secção de Choque, $\sigma(\mathrm{pb})$ & $\epsilon \cdot \sigma$ \\
\hline \hline$Z^{\prime} \rightarrow W W$ & 0.38 & $9.61 \cdot 10^{-1}$ & 0.36 \\
\hline$Z^{\prime} \rightarrow j j$ & $3.00 \cdot 10^{-4}$ & 5.82 & $1.75 \cdot 10^{-3}$ \\
\hline$Z^{\prime} \rightarrow t \bar{t}$ & $1.58 \cdot 10^{-2}$ & $2.90 \cdot 10^{-1}$ & $4.57 \cdot 10^{-3}$ \\
\hline$W W$ & 0.20 & $3.12 \cdot 10^{-6}$ & $6.38 \cdot 10^{-7}$ \\
\hline$Z Z$ & 0.18 & $3.81 \cdot 10^{-7}$ & $6.89 \cdot 10^{-8}$ \\
\hline$j j$ & $5.90 \cdot 10^{-4}$ & 1.48 & $8.73 \cdot 10^{-4}$ \\
\hline$t \bar{t}$ & 0.26 & $1.17 \cdot 10^{-5}$ & $3.04 \cdot 10^{-6}$ \\
\hline$j W^{+}$ & $1.25 \cdot 10^{-2}$ & $8.48 \cdot 10^{-4}$ & $1.06 \cdot 10^{-5}$ \\
\hline$j W^{-}$ & $1.23 \cdot 10^{-2}$ & $2.81 \cdot 10^{-4}$ & $3.46 \cdot 10^{-6}$ \\
\hline$j Z$ & $1.13 \cdot 10^{-2}$ & $3.58 \cdot 10^{-4}$ & $4.05 \cdot 10^{-6}$ \\
\hline
\end{tabular}

Tabela 6.8: Eficiência e secção de choque para $M_{Z^{\prime}}=3 \mathrm{TeV}$. 


\begin{tabular}{|c|c|c|c|}
\hline Processo & Eficiência, $\epsilon$ & Secção de Choque, $\sigma(\mathrm{pb})$ & $\epsilon \cdot \sigma$ \\
\hline \hline$Z^{\prime} \rightarrow W W$ & 0.32 & $2.80 \cdot 10^{-1}$ & $9.22 \cdot 10^{-2}$ \\
\hline$Z^{\prime} \rightarrow j j$ & $9.00 \cdot 10^{-5}$ & 1.06 & $9.51 \cdot 10^{-5}$ \\
\hline$Z^{\prime} \rightarrow t \bar{t}$ & $7.09 \cdot 10^{-3}$ & $3.10 \cdot 10^{-8}$ & $6.74 \cdot 10^{-9}$ \\
\hline$W W$ & 0.24 & $6.58 \cdot 10^{-2}$ & $4.67 \cdot 10^{-4}$ \\
\hline$Z Z$ & 0.22 & $3.10 \cdot 10^{-8}$ & $6.74 \cdot 10^{-9}$ \\
\hline$j j$ & $8.40 \cdot 10^{-4}$ & 0.10 & $8.54 \cdot 10^{-5}$ \\
\hline$t \bar{t}$ & 0.29 & $6.79 \cdot 10^{-7}$ & $1.98 \cdot 10^{-7}$ \\
\hline$j W^{+}$ & $1.49 \cdot 10^{-2}$ & $5.03 \cdot 10^{-5}$ & $7.50 \cdot 10^{-7}$ \\
\hline$j W^{-}$ & $1.54 \cdot 10^{-2}$ & $1.44 \cdot 10^{-5}$ & $2.23 \cdot 10^{-7}$ \\
\hline$j Z$ & $1.40 \cdot 10^{-2}$ & $2.04 \cdot 10^{-5}$ & $2.86 \cdot 10^{-7}$ \\
\hline
\end{tabular}

Tabela 6.9: Eficiência e secção de choque para $M_{Z^{\prime}}=4 \mathrm{TeV}$.

Com isso, podemos estudar o quanto nossos resultados estão dependentes dos parâmetros usados. Já vimos que para massas do $Z^{\prime}$ da ordem de $1 \mathrm{TeV}$ o comportamento do sinal e do algoritmo parecem ser estáveis, ou seja, não há grandes variações. Mas a secção de choque do sinal depende dos acoplamentos do $Z^{\prime}$ com o $W$ do MP e ainda do $Z^{\prime}$ com os quarks do MP. Podemos determinar quão pequenos podem ser esses acoplamentos para que ainda seja possível observar o novo bóson por esse canal de busca. Os acoplamentos usados nas simulações estão na tabela 6.10, sendo $g_{W W Z}$ o acoplamento do MP. Os acoplamentos do $Z^{\prime}$ com os quarks são unitários, tanto na parte axial quanto vetorial (diferentemente do MP) e o acoplamento com os bósons $W$ depende da massa do $Z^{\prime}$ para que a unitariedade do espalhamento seja garantida.

\begin{tabular}{|c|c|c|}
\hline$g_{W W Z^{\prime}}$ & $g_{Z^{\prime} q \bar{q}}$ & $g_{Z^{\prime} t \bar{t}}$ \\
\hline \hline$\frac{g_{W W Z} \cdot M_{Z}}{M_{Z^{\prime}} \cdot \sqrt{3}}$ & $1.0 \mathrm{GeV}$ & $1.0 \mathrm{GeV}$ \\
\hline
\end{tabular}

Tabela 6.10: Acoplamentos do $Z^{\prime}$ com o $W$ e os quarks do MP usados nas simulações.

Para declarar uma descoberta de nova física, é necessário que a significância estatística seja de ao menos $5 \sigma$. Isso significa que a discrepância entre os dados experimentais e o que é esperado pela teoria (fundos) é 5 vezes maior que o erro, de maneira que seria muito improvável que se trate de uma flutuação estatística. Pedindo então que tenhamos uma significância de $5 \sigma$ com uma luminosidade fixa podemos determinar quão pequenos os acoplamentos podem ser a fim de ainda vermos o sinal no $\mathrm{LHC}^{14}$ :

$$
\xi \frac{S}{\sqrt{B}}=5
$$

sendo $\xi$ um parâmetro multiplicativo dado por $\sigma=\xi \sigma_{\text {acoplamentos }}$, ou seja, a secção de choque do sinal pode ser $\xi$ vezes menor que a usada nas simulações e ainda seria possível obter um desvio de $5 \sigma . S$ é a soma de $\epsilon \cdot \sigma \cdot \mathcal{L}$ para todos os eventos identificados como sinal

\footnotetext{
${ }^{14} \mathrm{As}$ simulações foram feitas usando $14 \mathrm{TeV}$ de energia do centro de massa.
} 
e $B$ o análogo para os fundos, sendo $\mathcal{L}$ a luminosidade do acelerador. Para $\mathcal{L}=30 \mathrm{fb}^{-1}$ e $\mathcal{L}=100 \mathrm{fb}^{-1}$ os resultados obtidos foram são os da tabela 6.11 .

\begin{tabular}{|c|c|c|}
\hline$M_{Z^{\prime}}$ & $\xi\left(\mathcal{L}=30 \mathrm{fb}^{-1}\right)$ & $\xi\left(\mathcal{L}=100 \mathrm{fb}^{-1}\right)$ \\
\hline \hline $1 \mathrm{TeV}$ & $1.72 \cdot 10^{-3}$ & $9.44 \cdot 10^{-4}$ \\
\hline $2 \mathrm{TeV}$ & $1.43 \cdot 10^{-3}$ & $7.84 \cdot 10^{-4}$ \\
\hline $3 \mathrm{TeV}$ & $2.33 \cdot 10^{-3}$ & $1.28 \cdot 10^{-3}$ \\
\hline $4 \mathrm{TeV}$ & $2.90 \cdot 10^{-3}$ & $1.59 \cdot 10^{-3}$ \\
\hline
\end{tabular}

Tabela 6.11: Valores de $\xi$ para $\mathcal{L}=30 \mathrm{fb}^{-1}$ e $\mathcal{L}=100 \mathrm{fb}^{-1}$.

Com esses resultados para o parâmetro $\xi$ podemos determinar a razão $\frac{S}{B}$ para cada massa do $Z^{\prime}$ como na tabela 6.12 .

\begin{tabular}{|c|c|c|}
\hline$M_{Z^{\prime}}$ & $\xi \cdot \frac{S}{B}\left(\mathcal{L}=30 \mathrm{fb}^{-1}\right)$ & $\xi \cdot \frac{S}{B}\left(\mathcal{L}=100 \mathrm{fb}^{-1}\right)$ \\
\hline \hline $1 \mathrm{TeV}$ & $3.19 \cdot 10^{-2}$ & $1.75 \cdot 10^{-2}$ \\
\hline $2 \mathrm{TeV}$ & 0.23 & 0.13 \\
\hline $3 \mathrm{TeV}$ & 0.97 & 0.53 \\
\hline $4 \mathrm{TeV}$ & 3.10 & 1.70 \\
\hline
\end{tabular}

Tabela 6.12: Valores de $\xi \cdot \frac{S}{B}$ para $\mathcal{L}=30 \mathrm{fb}^{-1}$ e $\mathcal{L}=100 \mathrm{fb}^{-1}$ usando os valores de $\xi$ da tabela 6.11 .

Para valores de $\frac{S}{B}$ muito baixos, a confiabilidade dos resultados é pequena, uma vez que o conhecimento de todos os fundos deve ser muito preciso para que a análise seja válida, caso contrário podemos perder sinal ou ter sinais falsos. Quando se faz simulações estatísticas de resultados como no presente trabalho, sempre se está sujeito a flutuações do fundo. Até mesmo porque a teoria possui erros intrísecos, de modo que o conhecimento do valor de $B$ é na verdade flutuante: $B+\Delta B$. Com os resultados obtidos na tabela 6.12 , vemos que há espaço para uma flutuação do fundo e mesmo assima ainda será possível observar o $Z^{\prime}$, para massas maiores do novo bóson a secção de choque dos processos do MP ficam demasiadas pequenas facilitando ainda mais a análise, uma vez que a secção de choque dos processos do MP se tornam ainda menores. 


\section{Capítulo 7}

\section{Conclusões}

Apesar do enorme sucesso experimental do Modelo Padrão por várias décadas, há indícios de que ele não seja a teoria final na descrição das partículas elementares da natureza. Neste trabalho, vimos como teorias com dimensões extras podem estar relacionadas com o setor de quebra espontânea da simetria eletrofraca e como elas abordam a questão da unitariedade no espalhamento de bósons vetoriais longitudinais numa teoria sem Higgs.

Estudamos então a fenomenologia da observação de novos bósons vetoriais no LHC, em particular, o bóson $Z^{\prime}$ deixando um rastro puramente hadrônico no detector. Este sinal possui um enorme fundo de QCD, de maneira que o uso de técnicas de análise de subestrutura de jatos foi essencial para conseguirmos obter nossos resultados. Concluímos que o LHC tem um grande potencial de descoberta ou exclusão desse tipo de modelo, podendo fazer isso com $30 \mathrm{fb}^{-1}$ de luminosidade acumulada quando funcionar com $14 \mathrm{TeV}$.

Porém, esses resultados são somente preliminares, uma vez que diversos efeitos não foram considerados aqui. As simulações foram feitas somente a nível hadrônico e não a nível de detector e além disso, não consideramos nas simulações, alguns efeitos importantes como Pile-Up, Interações Múltiplas, entre outros. Ainda assim, os resultados sugerem que mesmo com a inclusão desses efeitos ainda será possível concluir algo sobre os modelos no LHC.

Em trabalhos futuros pretendemos tornar a análise ainda mais independente de modelos, usando diferentes larguras para o $Z^{\prime}$ e variando seus acoplamentos. Quanto ao algoritmo, iremos estudar o comportamento de outros algoritmos de recombinação, em particular o uso do C/A para agrupamento dos jatos. Usando as técnicas de análise de subestrutura apresentadas aqui, podemos ainda estudar o sinal semi-leptônico, onde um dos $W$ 's decae leptonicamente para tentar aumentar a eficiência de detecção mesmo quando para valores mais altos de seu mometo transverso.

É possível que as dificuldades apresentadas pela inclusão de efeitos como os citados sejam superadas utilizando as técnicas de grooming, descritas na secção 5.1.4. Somente com a análise de novas simulações que poderemos ter certeza se isso ocorrerá, mas o autor acredita que com o uso dessas novas ferramentas seja possível diminuir consideravelmente 
a interferência da radiação nas análises.

O algoritmo de identificação de partículas pesadas, como o $Z^{\prime}$ serve ainda para outros propósitos, não somente o estudo da fenomenologia de dimensões extras. O uso do FastJet facilitou muito a parte de programação, devido à grande quantidade de pacotes compatíveis que a comunidade científica tem elaborado. Isso torna o algoritmo bastante adaptável a outros tipos de buscas, pretende-se melhorar a eficiência de nosso método através da implementação de alguma das técnicas de Grooming, além disso podemos usar uma região menor de parâmetros $y^{2}$. Para diminuir a má-identificação devido aos pares $t \bar{t}$ iremos estudar a viabilidade de implementar as técnicas de identificação de tops estudadas em [64]. 


\section{Referências Bibliográficas}

[1] C. Quigg, "Gauge Theories Of Strong, Weak, And Electromagnetic Interactions", Westview Press, 1997.

[2] M. Peskin, D. Schroeder, "An Introduction To Quantum Field Theory", Westview Press, 1985.

[3] F. Halzen, A. Martin, "Quarks and Leptons: An Introductory Course in Modern Particle Physics", Wiley, 1984.

[4] Y. Nagashima, Y. Nambu, "Elementary Particle Physics", Wiley-VCH, 2010.

[5] D. J. Griffiths, "Introduction to Elementary Particles", Wiley-VCH, 2008.

[6] D. Perkins, "Introduction to High Energy Physics", Cambridge University Press, 2000.

[7] C. Itzykson, J-B. Zuber, "Quantum Field Theory", Dover Publications, 2006.

[8] B. Hartfield, "Quantum Field Theory of Point Particles and Strings", Westview Press, 1998.

[9] J. Donoghue, E. Golowich, B. R. Holstein, "Dynamics of the Standard Model", Cambridge Monographs on Particle Physics, Nuclear Physics and Cosmology, 1994.

[10] W. N. Cottingham, D. A. Greenwood. "An Introduction to the Standard Model of Particle Physics", Cambridge University Press, 2007.

[11] T-P. Cheng, L-F. Li, "Gauge Theory of elementary particle physics", Oxford Science Publications, 1988.

[12] H. Georgi, "Weak Interactions and Modern Particle Theory", Dover Publications, 2009.

[13] A. Pich, "The Standard Model of Electroweak Interactions", [arXiv:0705.4264v1 [hep-ph]] 
[14] S. Dawson, "Introduction to Electroweak Symmetry Breaking", [arXiv:hepph/9901280].

[15] S. L. Glashow, J.Iliopoulos, L. Maiani, "Weak Interactions with LeptonHadron Symmetry" Phys. Rev. D2 (1970) 1285.

[16] D. G. Netto, "Sinais de Produção de Novos Bósons Vetoriais no LHC", http://www.teses.usp.br/teses/disponiveis/43/43134/tde-26042010$141529 /$ pt-br.php.

[17] B. W. Lee, C. Quigg, H. B. Thacker, Phys. Rev. D 16, 1519 (1977).

[18] R. D .Peccei, H. Quinn, "Constraints Imposed by CP Conservation in the Presence of Instantons", Phys. Rev. D 16: (1977) 1791-1797.

[19] H.Baer, X. Tata, "Weak Scale Supersymmery - From Superfields to Scatering Events", Cambridge University Press.

[20] C. T. Hill and E. H. Simmons, "Strong Dynamics and Electroweak Symmetry Breaking", Phys. Rept. 381 (2003) 235-402; Erratum-ibid. 390 (2004) 553554 [arXiv: hep-ph/0203079].

[21] C. Csáki, "TASI Lectures on Extra Dimensions and Branes", [arXiv:hepph/0404096].

[22] M. Schmaltz, D. Tucker-Smith, "Little Higgs Review", Ann. Rev. Nucl. Part. Sci. 55: 229-270, 2005 [arXiv:hep-ph/0502182v1].

[23] LEP Electroweak Working Group, http://www.cern.ch/LEPEWWG/.

[24] ATLAS Collaboration, "Combined search for the Standard Model Higgs boson using up to $4.9 \mathrm{fb}^{-1}$ of pp collision data at $\sqrt{s}=7 \mathrm{TeV}$ with the ATLAS detector at the LHC", [arXiv:1202.1408v1 [hep-ex]].

[25] L. Randall, R. Sundrum, "A Large Mass Hierarchy from a Small Extra Dimension", Phys. Rev. Lett. 83 (1999): 3370-3373 [arXiv:hep-ph/9905221v1].

[26] C. Csáki, J.Hubisz and P. Meade, "TASI Lectures on Electroweak Symmetry Breaking from Extra Dimensions", [arXiv:hep-ph/0510275v1].

[27] A. Pérez-Lorenzana, "An Introduction to Extra Dimensions", J. Phys. Conf. Ser. 18 (2005) 224-269 [arXiv:hep-ph/0503177].

[28] P. M. de Aquino, "Física Além do Modelo Padrão em Teorias com Dimensões Extras", http://www.teses.usp.br/teses/disponiveis/43/43134/tde16122007-163258/pt-br.php. 
[29] R. S. Chivukula, Hong-Jian He, M. K., E. H. Simmons, M. Tanabashi, "General Sum Rules for WW Scattering in Higgsless Models: Equivalence Theorem and Deconstruction Identities", Phys. Rev. D 78: 095003, 2008 [arXiv:0808.1682v1 [hep-ph]].

[30] T. Sjostrand, S. Mrenna and P. Skands, JHEP 0605, 026 (2006) [arXiv: hep$\mathrm{ph} / 0603175]$.

[31] HERWIG 6.5, G. Corcella, I.G. Knowles, G. Marchesini, S. Moretti, K. Odagiri, P. Richardson, M.H. Seymour and B.R. Webber, JHEP 0101 (2001) 010 [hep-ph/0011363]; [hep-ph/0210213].

[32] G. Marchesini, B.R. Webber, G. Abbiendi, I.G. Knowles, M.H. Seymour and L. Stanco, Computer Phys. Commun. 67 (1992) 465.

[33] C. K. Vermilion, "Jet Substructure at the Large Hadron Collider: Harder, Better, Faster, Stronger", [arXiv:1101.1335v1 [hep-ph]].

[34] S. D. Ellis, C.K. Vermilion, J. R. Walsh, "Techniques for improved heavy particle searches with jet substructure", 10.1103/Phys. Rev. D 80051501 [arXiv:0903.5081v4 [hep-ph]].

[35] S. D. Ellis, C. K. Vermilion, J. R. Walsh,"Recombination Algorithms and Jet Substructure: Pruning as a Tool for Heavy Particle Searches", [arXiv:0912.0033v1 [hep-ph]].

[36] J. M. Butterworth, A. R. Davison, M. Rubin, G. P. Salam, "Jet substructure as a new Higgs search channel at the Large Hadron Collider", [arXiv:0810.0409v1 [hep-ph]].

[37] D. Krohn, J. Thaler, L-T. Wang, "Jet Trimming", 10.1007/JHEP 02 (2010) 084 [arXiv:0912.1342v2 [hep-ph]].

[38] G.P. Salam; "Towards Jetography" [arXiv: 0906.1833 [hep-ph]].

[39] S. D. Ellis, J. Huston, K. Hatakeyama, P. Loch, M. Tonnesmann; "Jets in Hadron-Hadron Collisions", Prog. Part.Nucl. Phys. 60:484-551, 2008 [arXiv: 0712.2447 [hep-ph]].

[40] A. Ali, G. Kramer; "Jets and QCD”, Eur. Phys. J. H 36, 245 - 326 (2011) [arXiv: 1012.2288 [hep-ph]].

[41] E. Halkiadakis, "Proceedings for TASI 2009 Summer School on "Physics of the Large and the Small": Introduction to the LHC experiments", [arXiv:1004.5564v1 [hep-ex]]. 
[42] Maxim Perelstein, "Collider Physics", [arXiv:1002.0274v2 [hep-ph]].

[43] G. P. Salam and G. Soyez, JHEP 0705 (2007) 086 [arXiv:0704.0292 [hep-ph]].

[44] W. Bartel et al. [JADE Collaboration], "Experimental Studies On Multi-Jet Production in $e^{+} e^{-}$Annihilation at Petra Energies", Z. Phys. C 8, 251 (1981).

[45] S. Bethke et al. [JADE Collaboration], "Experimental Investigation of the Energy Dependence of the Strong Coupling Strength", Phys. Lett. B 213 (1988) 235.

[46] S. Moretti, L. Lonnblad and T. Sjostrand, "New and old jet clustering algorithms for electron positron events", JHEP 9808 (1998) 001 [arXiv:hep$\mathrm{ph} / 9804296]$.

[47] S. Catani, Y. L. Dokshitzer, M. H. Seymour and B. R. Webber, "Longitudinally invariant $\mathrm{K}(\mathrm{t})$ clustering algorithms for hadron-hadron collisions", Nucl. Phys. B 406, 187 (1993).

[48] S. D. Ellis and D. E. Soper, "Successive Combination Jet Algorithm For Hadron Collisions," Phys. Rev. D 48, 3160 (1993) [hep-ph/9305266].

[49] S. Catani, Y. L. Dokshitzer, M. Olsson, G. Turnock and B. R. Webber, "New clustering algorithm for multi - jet cross-sections in $e^{+} e^{-}$annihilation," Phys. Lett. B 269, 432 (1991);

[50] D. Krohn, J. Thaler, L-T. Wang, "Jets with variable $R$ ", [arXiv:0903.0392v1 [hep-ph]].

[51] M. Cacciari, G.P. Salam and G. Soyez, "Dispelling the $N^{3}$ myth for the Kt jet-finder", Phys.Lett.B 641:57-61,2006 [arXiv:1111.6097 ].

[52] M. Cacciari, G. P. Salam, G. Soyez, "FastJet user manual", [arXiv:1111.6097v1 [hep-ph]].

[53] Yu.L. Dokshitzer, G.D. Leder, S. Moretti, B.R. Webber, "Better Jet Clustering Algorithms", JHEP 9708:001,1997 [arXiv:hep-ph/9707323v2].

[54] M. Wobisch, T. Wengler, "Hadronization Corrections to Jet Cross Sections in Deep-Inelastic Scattering" [arXiv:hep-ph/9907280v1].

[55] J. E. Huth et al., "Toward a standardization of jet definitions," FNAL-C90-249-E, published in the proceedings of the 1990 Summer Study on High Energy Physics, Research Directions for the Decade, Snowmass, Colorado, June 25 - July 13, 1990. 
[56] M. Cacciari, G. P. Salam and G. Soyez, "The anti-kt jet clustering algorithm," JHEP 0804 (2008) 063 [arXiv:0802.1189 [hep-ph]].

[57] T. Plehn, M. Spannowsky, M. Takeuchi, D. Zerwas, "Stop Reconstruction with Tagged Tops", JHEP 1010:078,2010 [arXiv:1006.2833v2 [hep-ph]].

[58] D. E. Kaplan, K. Rehermann, M. D. Schwartz, B. Tweedie, "Top-tagging: A Method for Identifying Boosted Hadronic Tops", Phys. Rev. Lett. 101:142001,2008 [arXiv:0806.0848v2 [hep-ph]].

[59] M. H. Seymour, "Searches for new particles using cone and cluster jet algorithms: A Comparative study," Z. Phys. C 62 (1994) 127.

[60] J. M. Butterworth, B. E. Cox and J. R. Forshaw, "W W scattering at the LHC," Phys. Rev. D 65 (2002) 096014 [arXiv:hep-ph/0201098].

[61] Y. Cui, Z. Han, M. Schwartz, "W-jet Tagging: Optimizing the Identification of Boosted Hadronically-Decaying $W$ Bosons", Phys. Rev. D 83:074023, 2011 [arXiv:1012.2077v2 [hep-ph]].

[62] A. Alves, O.J.P. Eboli, D. Goncalves, M.C. Gonzalez-Garcia, J. K. Mizukoshi, "Signals for New Spin-1 Resonances in Electroweak Gauge Boson Pair Production at the LHC", Phys. Rev. D. 80, 073011 (2009) [arXiv:0907.2915v1 [hep-ph]].

[63] J. Alwall, P. Demin, S. de Visscher, R. Frederix, M. Herquet, F. Maltoni, T. Plehn, D. L. Rainwater, T. Stelzer, "MadGraph/MadEvent v4: The New Web Generation", JHEP 0709:028, (2007) [arXiv:0706.2334v1 [hep-ph]].

[64] C. M. Mello, "Estudo da detecção de quarks top no LHC", dissertação de mestrado disponível no banco de teses da USP. 